Comparative Effectiveness Review

Number 193

\title{
Management of Suspected Opioid Overdose with Naloxone by Emergency Medical Services Personnel
}


Number 193

\title{
Management of Suspected Opioid Overdose With Naloxone by Emergency Medical Services Personnel
}

\author{
Prepared for: \\ Agency for Healthcare Research and Quality \\ U.S. Department of Health and Human Services \\ 5600 Fishers Lane \\ Rockville, MD 20857 \\ www.ahrq.gov \\ Contract No. 290-2015-00009-I \\ Prepared by: \\ Pacific Northwest Evidence-based Practice Center \\ Portland, OR \\ Investigators: \\ Roger Chou, M.D. \\ P. Todd Korthuis, M.D., M.P.H. \\ Dennis McCarty, Ph.D. \\ Phillip Coffin, M.D., M.I.A. \\ Jessica Griffin, M.S. \\ Cynthia Davis-O’Reilly, B.S. \\ Sara Grusing, B.A. \\ Mohamud Daya, M.D., M.S.
}

AHRQ Publication No. 17(18)-EHC025-EF

November 2017 


\section{Key Messages}

\section{Purpose of Review}

To determine optimal doses, routes of administration, and dosing strategies of naloxone for suspected opioid overdose in out-of-hospital settings, and whether transport to a hospital following successful opioid overdose reversal with naloxone is necessary.

Key Messages

- Higher concentration intranasal naloxone may be similarly effective and safe compared with intramuscular naloxone, but the available studies did not evaluate formulations approved by the Food and Drug Administration.

- While field administration of naloxone is generally effective in reversing opioid overdose, there is not strong evidence concerning differences in effectiveness between doses or routes of administration.

- More research is needed to determine optimal doses of naloxone, appropriate timing of repeat dosing, and whether it is necessary to dose patients to full consciousness.

- More research is needed to determine whether transporting patients to a hospital after successful reversal of overdose is necessary. 
This report is based on research conducted by the Pacific Northwest Evidence-based Practice Center under contract to the Agency for Healthcare Research and Quality (AHRQ), Rockville, MD (Contract No. 290-2015-00009-I). The findings and conclusions in this document are those of the authors, who are responsible for its contents; the findings and conclusions do not necessarily represent the views of AHRQ. Therefore, no statement in this report should be construed as an official position of AHRQ or of the U.S. Department of Health and Human Services.

\section{None of the investigators have any affiliations or financial involvement that conflicts with the material presented in this report.}

The information in this report is intended to help health care decisionmakers-patients and clinicians, health system leaders, and policymakers, among others-make well-informed decisions and thereby improve the quality of health care services. This report is not intended to be a substitute for the application of clinical judgment. Anyone who makes decisions concerning the provision of clinical care should consider this report in the same way as any medical reference and in conjunction with all other pertinent information, i.e., in the context of available resources and circumstances presented by individual patients.

This report is made available to the public under the terms of a licensing agreement between the author and the Agency for Healthcare Research and Quality. This report may be used and reprinted without permission except those copyrighted materials that are clearly noted in the report. Further reproduction of those copyrighted materials is prohibited without the express permission of copyright holders.

AHRQ or U.S. Department of Health and Human Services endorsement of any derivative products that may be developed from this report, such as clinical practice guidelines, other quality enhancement tools, or reimbursement or coverage policies, may not be stated or implied.

This report may periodically be assessed for the currency of conclusions. If an assessment is done, the resulting surveillance report describing the methodology and findings will be found on the Effective Health Care Program Web site at www.effectivehealthcare.ahrq.gov. Search on the title of the report.

Persons using assistive technology may not be able to fully access information in this report. For assistance contact EffectiveHealthCare@ahrq.hhs.gov.

Suggested citation: Chou R, Korthuis PT, McCarty D, Coffin P, Griffin J, Davis-O’Reilly C, Grusing S, Daya M. Management of Suspected Opioid Overdose With Naloxone by Emergency Medical Services Personnel. Comparative Effectiveness Review No. 193. (Prepared by the Pacific Northwest Evidence-based Practice Center under Contract No. 290-2015-00009-I.) AHRQ Publication No. 17(18)-EHC025-EF. Rockville, MD: Agency for Healthcare Research and Quality; November 2017. www.effectivehealthcare.ahrq.gov/reports/final.cfm. DOI: https://doi.org/10.23970/AHRQEPCCER193. 


\section{Preface}

The Agency for Healthcare Research and Quality (AHRQ), through its Evidence-based Practice Centers (EPCs), sponsors the development of evidence reports and technology assessments to assist public- and private-sector organizations in their efforts to improve the quality of health care in the United States. The National Highway Traffic Safety Administration (NHTSA) requested and provided funding for this report.

The reports and assessments provide organizations with comprehensive, evidence-based information on common medical conditions and new health care technologies and strategies. They also identify research gaps in the selected scientific area, identify methodological and scientific weaknesses, suggest research needs, and move the field forward through an unbiased, evidence-based assessment of the available literature. The EPCs systematically review the relevant scientific literature on topics assigned to them by AHRQ and conduct additional analyses when appropriate prior to developing their reports and assessments.

To bring the broadest range of experts into the development of evidence reports and health technology assessments, AHRQ encourages the EPCs to form partnerships and enter into collaborations with other medical and research organizations. The EPCs work with these partner organizations to ensure that the evidence reports and technology assessments they produce will become building blocks for health care quality improvement projects throughout the Nation. The reports undergo peer review and public comment prior to their release as a final report.

AHRQ expects that the EPC evidence reports and technology assessments, when appropriate, will inform individual health plans, providers, and purchasers as well as the health care system as a whole by providing important information to help improve health care quality.

If you have comments on this evidence report, they may be sent by mail to the Task Order Officers (TOOs) named below at: Agency for Healthcare Research and Quality, 5600 Fishers Lane, Rockville, MD 20857, or by email to epc@ahrq.hhs.gov.

Gopal Khanna, M.B.A.

Director

Agency for Healthcare Research and Quality

Stephanie Chang, M.D., M.P.H.

Director

Evidence-based Practice Center Program

Center for Evidence and Practice Improvement Agency for Healthcare Research and Quality
Arlene S. Bierman, M.D., M.S.

Director

Center for Evidence and Practice Improvement

Agency for Healthcare Research and Quality

David Niebuhr, M.D., M.P.H., M.Sc.

Task Order Officer

Center for Evidence and Practice

Improvement

Agency for Healthcare Research and Quality

Laura Pincock, Pharm.D., M.P.H.

Task Order Officer

Center for Evidence and Practice

Improvement

Agency for Healthcare Research and Quality 


\section{Acknowledgments}

The authors gratefully acknowledge the following individuals for their contributions to this project: Elaine Graham, M.L.S.; Tracy Dana, M.L.S.; Leah Williams, BS; and Hyon Hildebrant, B.A.

\section{Key Informants}

Key Informants were not involved in the development of this report.

\section{Technical Expert Panel}

In designing the study questions and methodology at the outset of this report, the EPC consulted several technical and content experts. Broad expertise and perspectives were sought. Divergent and conflicting opinions are common and perceived as healthy scientific discourse that results in a thoughtful, relevant systematic review. Therefore, in the end, study questions, design, methodologic approaches, and/or conclusions do not necessarily represent the views of individual technical and content experts.

Technical Experts must disclose any financial conflicts of interest greater than $\$ 10,000$ and any other relevant business or professional conflicts of interest. Because of their unique clinical or content expertise, individuals with potential conflicts may be retained. The TOO and the EPC work to balance, manage, or mitigate any potential conflicts of interest identified.

The list of Technical Experts who provided input to this report follows:

Caleb Banta-Green, Ph.D., M.P.H., M.S.W.

University of Washington

Seattle, WA

Michael Dailey,* M.D., FACEP, FAEMS

Albany Medical College

Albany, NY

Corey Davis,* J.D., M.S.P.H., EMT-B

Network for Public Health Law

Los Angeles, CA

James J. Gasper,* Pharm.D., BCPP

California Department of Healthcare Services

Sacramento, CA

Christopher Jones, Pharm.D., Ph.D.

U.S. Department of Health and Human Services

Washington, DC 
Scott Russell,* R.N., B.S.N., EMT-P

Metrohealth

Cleveland, $\mathrm{OH}$

Karl Sporer, M.D.

University of California, San Francisco

San Francisco, CA

Sharon Stancliff,* M.D.

Harm Reduction Coalition

New York, NY

Gregory Terman,* M.D., Ph.D.

University of Washington

Seattle, WA

U.S. Food and Drug Administration*

Center for Drug Evaluation and Research

Office of New Drugs

Division of Analgesia, Anesthesia, and Addiction Products

Silver Spring, MD

Alexander Walley, M.D., M.Sc.

Boston University

Boston, MA

*Provided input on Draft Report.

\section{Peer Reviewers}

Prior to publication of the final evidence report, EPCs sought input from independent Peer reviewers without financial conflicts of interest. However, the conclusions and synthesis of the scientific literature presented in this report do not necessarily represent the views of individual reviewers.

Peer Reviewers must disclose any financial conflicts of interest greater than $\$ 10,000$ and any other relevant business or professional conflicts of interest. Because of their unique clinical or content expertise, individuals with potential nonfinancial conflicts may be retained. The TOO and the EPC work to balance, manage, or mitigate any potential nonfinancial conflicts of interest identified.

The list of Peer Reviewers follows:

Steven Aks, D.O., FACMT, FACEP

Toxicon Consortium,

Cook County Health and Hospitals System

Chicago, IL 
Jeffrey Bratberg, Pharm.D.

University of Rhode Island

Kingston, RI

Christian Martin-Gill, M.D., M.P.H.

University of Pittsburgh

Pittsburgh, PA

Jennifer Plumb, M.D., M.P.H.

University of Utah

Salt Lake City, UT

Ken Sternig, M.S.-E.H.S., B.S.N., Paramedic

Office of Emergency Management

Milwaukee, WI 


\section{Management of Suspected Opioid Overdose With Naloxone by Emergency Medical Services Personnel}

\section{Structured Abstract}

Objectives. To compare different routes, doses, and dosing strategies of naloxone administration for suspected opioid overdose by emergency medical services (EMS) personnel in field settings, and to compare effects of transport to a health care facility versus nontransport following successful reversal of opioid overdose with naloxone.

Methods. Four databases were searched through September 2017. Additional studies were identified from reference lists and technical experts. We included randomized controlled trials (RCTs) and cohort studies comparing different naloxone routes of administration, doses, or dosing strategies and on effects of transport or nontransport following successful reversal of opioid overdose with naloxone. Two investigators independently applied prespecified criteria to rate study quality. The strength of evidence was determined based on the overall risk of bias, consistency, directness, precision, and reporting bias. Main outcomes were mortality, reversal of opioid overdose symptoms, time to reversal of symptoms, recurrence of overdose symptoms, and harms.

Results. Thirteen studies met inclusion criteria. Three RCTs and four cohort studies compared different routes of administration. Two trials compared intranasal (IN) with intramuscular (IM) naloxone administration (strength of evidence [SOE] for all outcomes: low). While $2 \mathrm{mg}$ of a higher concentration formulation of IN naloxone $(2 \mathrm{mg} / 1 \mathrm{~mL})$ is similar in efficacy to $2 \mathrm{mg}$ of IM naloxone, $2 \mathrm{mg}$ of a lower concentration formulation of IN naloxone $(2 \mathrm{mg} / 5 \mathrm{~mL})$ is less effective than the same dose IM but associated with decreased risk of agitation and/or irritation. The $2 \mathrm{mg} / 5 \mathrm{~mL}$ formulation of IN naloxone studied in this trial is lower than concentrations used in the United States. In both trials, IN naloxone was associated with increased likelihood of rescue naloxone use.

Although one RCT and two observational studies evaluated intravenous (IV) versus IN naloxone, evidence was insufficient to determine comparative benefits and harms because of methodological limitations and poor applicability to U.S. EMS settings (SOE: insufficient). There was insufficient evidence from two observational studies to compare parenteral routes of administration (IM, IV, or subcutaneous).

No study compared outcomes of patients transported versus not transported following successful reversal of opioid overdose with naloxone. Six studies reported low rates of deaths and serious adverse events ( $0 \%$ to $1.25 \%$ ) in patients not transported to a hospital after successful naloxone treatment but used an uncontrolled design and had other methodological limitations (SOE: insufficient).

Limitations. Few studies met inclusion criteria, all studies had methodological limitations, and no study evaluated naloxone auto-injectors or IN naloxone formulations recently approved by the U.S. Food and Drug Administration (FDA).

Conclusions. Low-strength evidence suggested that higher concentration IN naloxone (2 mg/1 $\mathrm{mL}$ ) is similar in efficacy to IM naloxone (2 mg), with no difference in adverse events. Research is needed on the comparative effectiveness of the FDA-approved naloxone auto-injectors $(0.4$ $\mathrm{mg}$ and $2 \mathrm{mg}$ ) and highly concentrated (4 mg/0.1 mL and $2 \mathrm{mg} / 0.1 \mathrm{~mL}$ ) IN naloxone 
reformulation, different doses, and dosing strategies. Uncontrolled studies suggest that nontransport of patients following successful naloxone reversal of overdose is associated with a low rate of serious harms, but patients were probably at low risk for such events, and no study evaluated risk of transport versus nontransport. 


\section{Contents}

Executive Summary ............................................................................................................................... ES-1

Introduction ..................................................................................................................................... 1

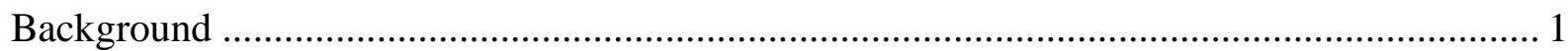

Nature and Burden of Opioid Overdose ............................................................................ 1

Field Treatment of Suspected Opioid Overdose With Naloxone ............................................. 1

Rationale for Review ……………………………….............................................. 3

Scope and Key Questions ............................................................................................. 3

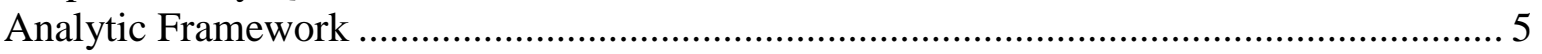

Methods.............................................................................................................................................. 6

Topic Refinement and Review Protocol .............................................................................. 6

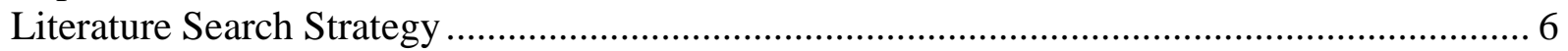

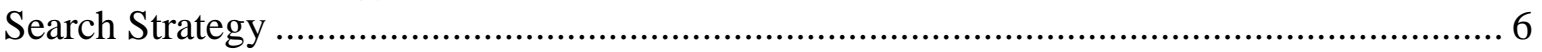

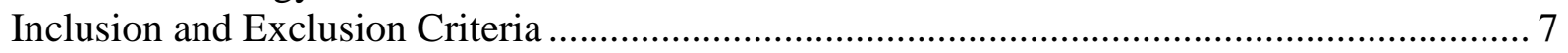

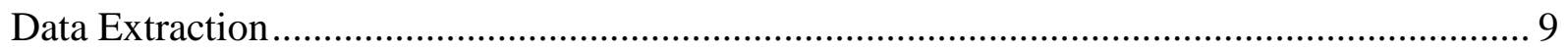

Risk of Bias Assessment of Individual Studies........................................................................ 9

Assessing Research Applicability .................................................................................. 10

Data Synthesis and Rating the Body of Evidence ................................................................. 10

Peer Review and Public Commentary ................................................................................ 11

Results ........................................................................................................................................................... 12

Results of Literature Searches .......................................................................................... 12

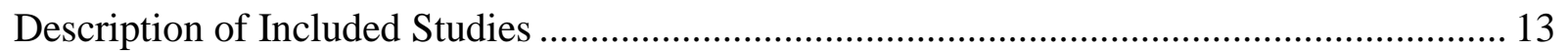

Key Question 1: For patients with confirmed or suspected opioid overdose, what are the comparative benefits and harms of out-of-hospital administration of naloxone by EMS personnel using intravenous, intramuscular, subcutaneous, and intranasal routes of

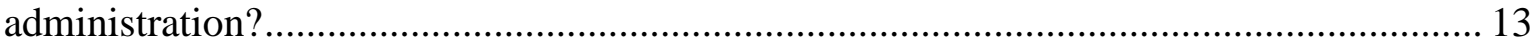

Key Question 1a: For patients with confirmed or suspected opioid overdose who receive naloxone in the out-of-hospital setting from EMS personnel, what are the comparative benefits and harms of different intravenous, intramuscular, subcutaneous, or intranasal doses of naloxone?

Key Question 2: For patients with confirmed or suspected opioid overdose in out-ofhospital settings, what are the comparative benefits and harms of titration of naloxone administered by EMS personnel until the patient resumes sufficient spontaneous respiratory effort versus until the patient regains consciousness? .......................................................... 19

Key Question 3: For patients with confirmed or suspected opioid overdose in out-ofhospital settings treated with multiple doses of naloxone (including patients who do not improve after an initial dose of intranasal naloxone), what are the effects on benefits and harms of differences in timing of repeat dosing?

Key Question 4: For patients with confirmed or suspected opioid overdose in out-ofhospital settings who regain sufficient spontaneous respiratory effort and are alert and oriented after naloxone administration by EMS personnel, what are the benefits and harms of transporting patients to a health care facility versus nontransport?................................... 20

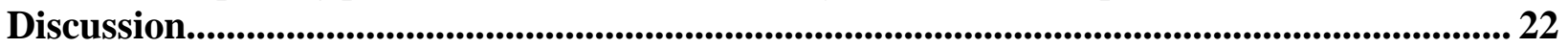

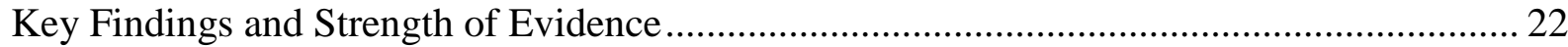

Findings in Relationship to What Is Already Known ............................................................ 23

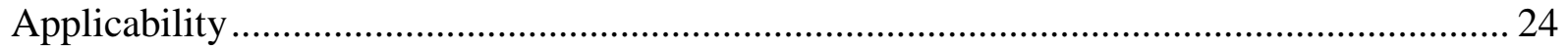


Implications for Clinical and Policy Decisionmaking ................................................... 25

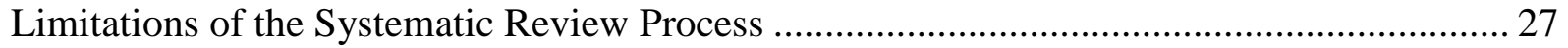

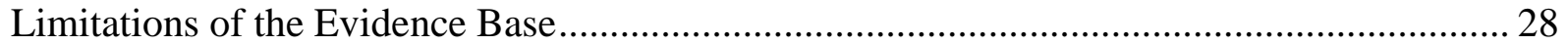

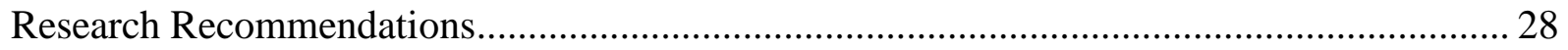

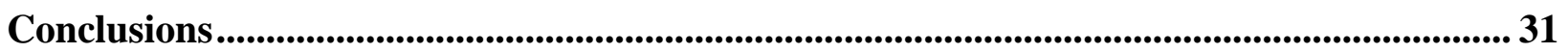

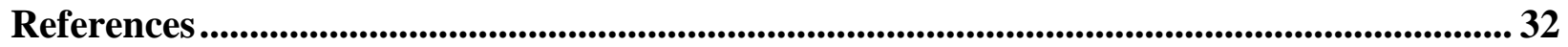

Tables

Table A. Naloxone: Dose and route of administration ...................................................... ES-4

Table 1. Naloxone: Dose and route of administration ........................................................... 7

Table 2. Characteristics and results of randomized controlled trials comparing routes of naloxone

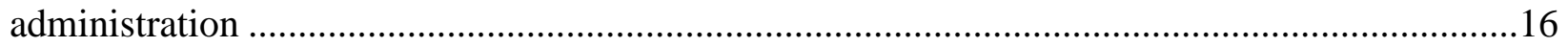

Table 3. Characteristics and results of cohort studies comparing routes of naloxone

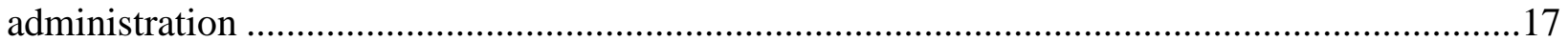

Table 4. Characteristics and results of uncontrolled studies of patients not transported to hospitals

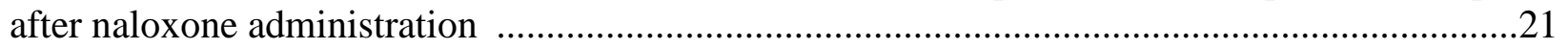

Figures

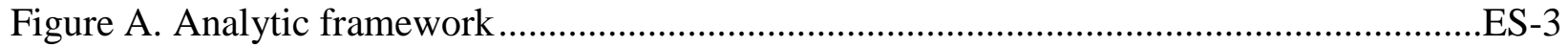

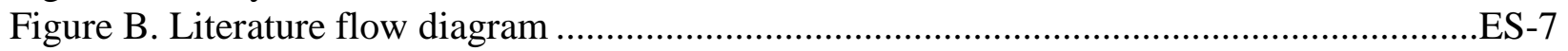

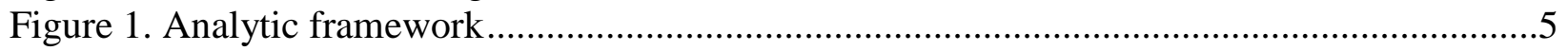

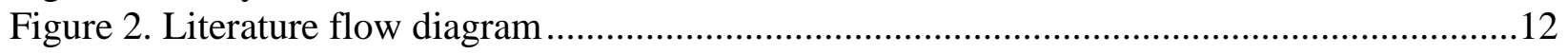

\section{Appendixes}

Appendix A. Search Strategies

Appendix B. Included Studies List

Appendix C. Excluded Studies List

Appendix D. Data Abstraction

Appendix E. Risk of Bias

Appendix F. Strength of Evidence 


\section{Executive Summary}

\section{Background}

\section{Nature and Burden of Opioid Overdose}

Addiction and overdoses associated with prescription and illicit opioids have been characterized by the U.S. Department of Health and Human Services as a national crisis. ${ }^{1}$ Since 2000, the rate of overdose deaths involving opioids has increased four-fold. ${ }^{2,3}$ Drug overdose deaths are now the leading cause of injury-related death in the United States. ${ }^{4}$ Overdoses due to opioids cause respiratory depression that can progress to cardiac arrest if untreated. In 2015, the number of drug overdose deaths involving prescription or illicit opioids exceeded 33,000, the highest number on record. ${ }^{3}$ Of recent concern is whether dosing guidelines are sufficient for reversing overdose related to highly potent synthetic opioids (e.g., fentanyl and fentanyl analogues).

\section{Field Treatment of Suspected Opioid Overdose With Naloxone}

Naloxone can be administered by the intravenous (IV), intramuscular (IM), subcutaneous (SC), intranasal (IN), endotracheal (ET), nebulized/inhalational, buccal, or sublingual routes. ${ }^{10}$ The U.S. Food and Drug Administration (FDA) approved a handheld naloxone IM or SC autoinjector in $2014^{11}$ and a new IN formulation and delivery device in $2015 ;{ }^{12}$ both administer a preset dose. With IN administration of highly concentrated naloxone using a preloaded single dose device, there is no risk of needle stick injury. Both the auto-injector and IN formulation are designed for ease of administration even by individuals with limited or no health care training. Off-label administration of IN naloxone in a less concentrated formulation using an improvised intranasal device is also common. Naloxone has been shown to be effective for reversal of opioid overdose across various routes of administration and doses. ${ }^{13,14}$ Naloxone may precipitate withdrawal symptoms. ${ }^{15}$ While uncomfortable, withdrawal symptoms are generally not serious or life-threatening and generally short-lived; the half-life of naloxone is about 30 minutes. Postwithdrawal agitation following naloxone administration may put the person administering the naloxone at increased risk for injury. ${ }^{16,17}$

When responding to opioid overdoses, early intervention is critical to prevent death and other complications. ${ }^{18}$ Emergency medical services (EMS) personnel are often involved in management of potential opioid overdoses. Management of opioid overdoses by EMS personnel includes airway management and continuous assessment of oxygenation and ventilation, along with administration of naloxone. ${ }^{19}$ According to the National EMS Information System database, the number of EMS encounters for suspected opioid overdose has increased, ${ }^{20}$ with nearly 160,000 doses of naloxone administered by EMS personnel in $2014 .^{21}$ Regulations vary, however, with regard to whether EMS personnel with different levels of training are permitted to administer naloxone. Naloxone administration is not currently within the National EMS Scope of Practice Model for EMTs and EMRs, which was last updated in 2007, ${ }^{22}$ prior to the introduction of newer naloxone formulations and availability of newer evidence on the benefits of field use of naloxone.

Although a number of recommendations, guidelines, and protocols are available to inform out-of-hospital management of opioid overdose patients, including naloxone use, guidance varies across these documents, and there are uncertainties in a number of areas. ${ }^{23-25}$ These include the 
optimal route of administration, the optimal dose for different routes of administration, optimal dosing strategies, and appropriate training levels for EMS personnel who are permitted to administer naloxone.

The purpose of this systematic review is to synthesize the evidence on naloxone route of administration and dosing for suspected opioid overdose in out-of-hospital settings, and on the need for transport to a hospital following successful opioid overdose reversal with naloxone; the review is intended to inform development of evidence-based guidelines on EMS management of suspected opioid overdose with naloxone and potentially inform an update to the National EMS Scope of Practice Model regarding naloxone use across EMS training levels.

\section{Scope and Key Questions}

The report addresses the following Key Questions.

Key Question 1: For patients with confirmed or suspected opioid overdose, what are the comparative benefits and harms of out-of-hospital administration of naloxone by EMS personnel using intravenous, intramuscular, subcutaneous, and intranasal routes of administration?

1a. For patients with confirmed or suspected opioid overdose who receive naloxone in the out-of-hospital setting from EMS personnel, what are the comparative benefits and harms of different intravenous, intramuscular, subcutaneous, or intranasal doses of naloxone?

Key Question 2: For patients with confirmed or suspected opioid overdose in out-of-hospital settings, what are the comparative benefits and harms of titration of naloxone administered by EMS personnel until the patient resumes sufficient spontaneous respiratory effort versus until the patient regains consciousness?

Key Question 3: For patients with confirmed or suspected opioid overdose in out-of-hospital settings treated with multiple doses of naloxone (including patients who do not improve after an initial dose of intranasal naloxone), what are the effects on benefits and harms of differences in timing of repeat dosing?

Key Question 4: For patients with confirmed or suspected opioid overdose in out-of-hospital settings who regain sufficient spontaneous respiratory effort and are alert and oriented after naloxone administration by EMS personnel, what are the benefits and harms of transporting patients to a health care facility versus nontransport?

The analytic framework (Figure A) shows the target population, interventions, and health outcomes examined; the Key Questions are numbered and indicated in the framework. We 
focused on use of IN, IM, and IV naloxone; these are the formulations of naloxone most commonly used for reversal of suspected opioid overdose in the field.

Figure A. Analytic framework

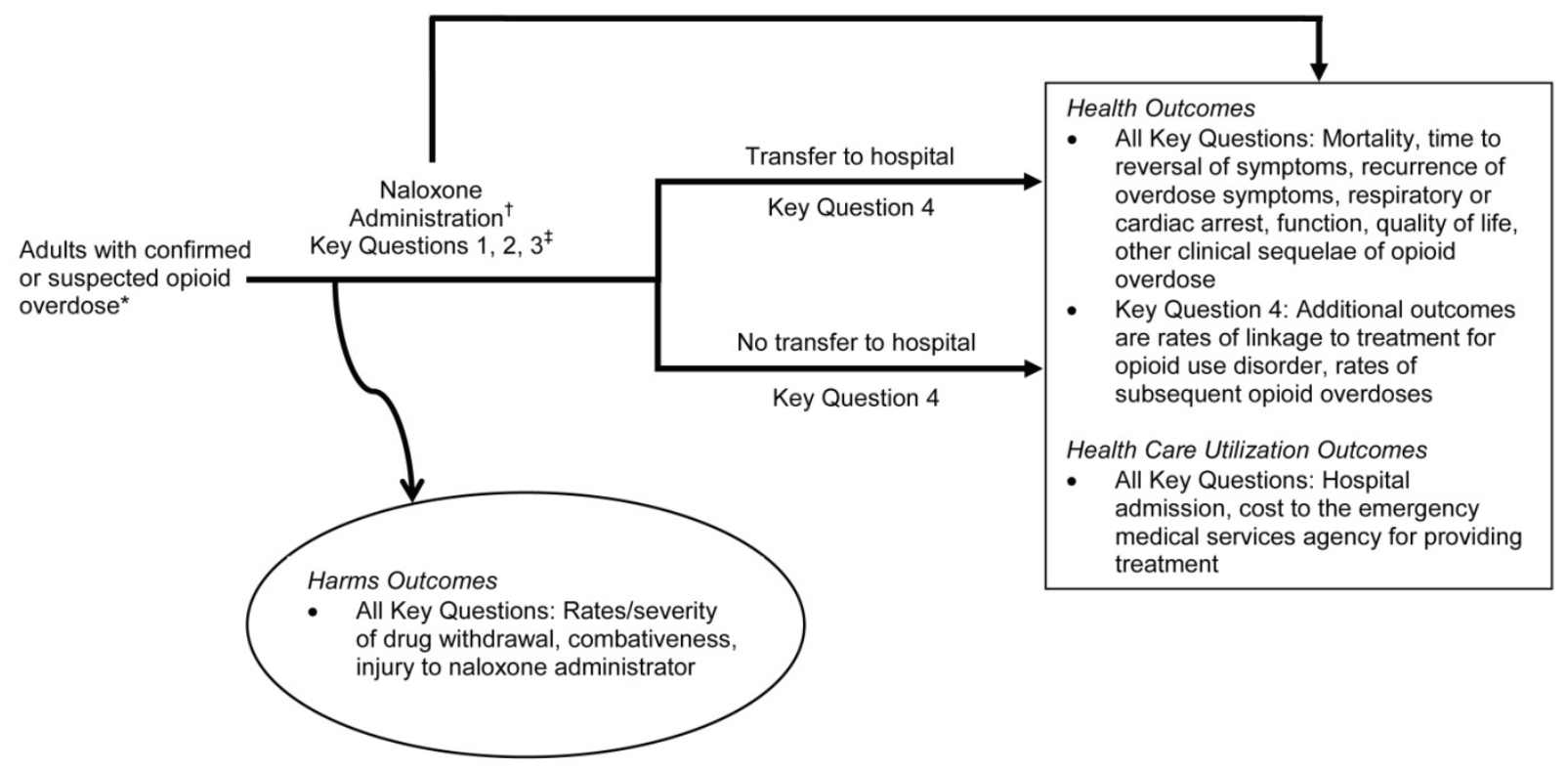

\begin{abstract}
* Patients with confirmed or suspected opioid overdose who exhibit altered mental status, miosis, or respiratory distress and who are treated in the out-of-hospital setting by emergency medical services personnel

†Administration of naloxone hydrochloride via the nasal, intravenous, intramuscular, or subcutaneous injection (including the naloxone auto-injector)

¥ Key Question 1 addresses comparisons involving route of administration and dose; Key Question 2 addresses comparisons involving dose titration to varying degrees of return of consciousness (intermediate outcome)
\end{abstract}

\title{
Methods
}

The final protocol was posted on the AHRQ Web site on November 30, 2016, at: https://www.effectivehealthcare.ahrq.gov/search-for-guides-reviews-andreports/?pageaction=displayproduct\&productid $=2360$ and registered in PROSPERO (CRD42016053891).

\section{Literature Search Strategy}

A research librarian conducted searches in Ovid MEDLINE (1946-August Week 2 2016), PsycINFO, the Cochrane Central Register of Controlled Trials (CCRCT), and the Cumulative Index to Nursing and Allied Health Literature (CINAHL). We did not apply search date restrictions and updated searches were conducted through September 2017. The Agency for Healthcare Research and Quality (AHRQ) Evidence-based Practice Center (EPC) Scientific Resource Center (SRC) sent email notification to relevant stakeholders about the opportunity to submit Scientific Information Packets (SIPs) via the Effective Health Care (EHC) Web site for naloxone.

We also hand-searched reference lists of relevant studies, searched for unpublished or ongoing studies in ClinicalTrials.gov, contacted representatives of federal agencies involved in naloxone or opioid overdose research (CDC, NIDA, SAMHSA), and reviewed materials presented at a recent FDA meeting ${ }^{26}$ on naloxone dosing. ${ }^{27-32}$ 


\section{Inclusion and Exclusion Criteria}

We developed pre-established criteria for inclusion and exclusion of studies based on the Key Questions and the populations, interventions, comparators, outcomes, timing, types of studies, and setting (PICOTS) approach, in accordance with the AHRQ Methods Guide for Effectiveness and Comparative Effectiveness Reviews. ${ }^{33}$ Inclusion and exclusion criteria are described below.

\section{Population(s)}

- Include: Patients with confirmed or suspected opioid overdose who exhibit altered mental status, miosis, or respiratory depression and who are treated in the out-of-hospital setting by EMS personnel

o Also include studies of naloxone administration in out-of-hospital settings by nonEMS personnel (e.g., police, other first responders, laypeople), which may inform optimal dosing strategies in EMS personnel

o For Key Questions 1 and 1a, also include studies of patients treated in emergency department (ED) settings by ED personnel.

\section{Interventions}

- For Key Questions 1-3: See Table A for included naloxone formulations.

Table A. Naloxone: Dose and route of administration

\begin{tabular}{|l|l|}
\hline Included Drug & Dose and Route of Administration \\
\hline Naloxone & $\bullet \quad$ Auto-injector, intramuscular (IM) \\
$0 \quad 0.4 \mathrm{mg} / 0.4 \mathrm{~mL},{ }^{*} 2 \mathrm{mg} / 0.4 \mathrm{~mL}$ \\
Nasal spray, intranasal (IN) \\
o Single dose intranasal device: $4 \mathrm{mg} / 0.1 \mathrm{~mL}, 2 \mathrm{mg} / 0.1 \mathrm{~mL}$ \\
o Improvised intranasal device: $2 \mathrm{mg} / 2 \mathrm{~mL}^{\dagger}$ \\
\\
Injection, intravenous, intramuscular or subcutaneous \\
$0 \quad 0.4 \mathrm{mg} / \mathrm{mL}, 1 \mathrm{mg} / \mathrm{mL}, 2 \mathrm{mg} / \mathrm{mL}$ \\
\hline
\end{tabular}

* Manufacturer has stopped production of $0.4 \mathrm{mg} / 0.4 \mathrm{~mL} \mathrm{IM}$

$\dagger$ Formulation not currently approved by the FDA for intranasal administration

- Potential modifiers of interventions: Based on training and background of the person administering naloxone

- For Key Question 4: Transport to health care facility

- Exclude: Naloxone in combination with other medications (e.g., buprenorphine/ naloxone)

\section{Comparators}

- Key Question 1: Injection (intramuscular, subcutaneous or intravenous) versus intranasal route of administration

- Key Question 1a: Comparisons of different doses of intranasal, intramuscular, and intravenous naloxone

- Key Question 2: Titration of patients until they resume spontaneous respiration but have some residual sedation/altered mental status versus dosing of patient until they resume spontaneous respiration and are awake and alert

- Key Question 3: Comparison of differences in timing of repeat dosing 
- Key Question 4: Transport of patients following treatment of opioid overdose with naloxone to a health care facility versus nontransport

\section{Outcomes}

- All Key Questions: Mortality, time to reversal of symptoms, recurrence of overdose symptoms, respiratory or cardiac arrest, function, quality of life, other clinical sequelae of opioid overdose; health care utilization indicators (e.g., hospital admission, cost to the EMS agency for providing treatment); and adverse effects and other harms (such as rates/severity of drug withdrawal, combativeness, injury to administrator of naloxone)

- Key Question 4: Additional outcomes are rates of linkage to treatment for opioid use disorder and rates of subsequent opioid overdoses

\section{Timing}

- No restrictions on timing of followup

\section{Settings}

- Include: Out-of-hospital setting and ED setting (for Key Questions 1 and 1a). The addition of studies conducted in ED settings was a protocol modification for Key Questions 1 and 1a, due to few randomized controlled trials (RCTs) conducted in field settings.

- Exclude: Inpatient, clinic, or ED setting (for Key Questions other than 1 and 1a)

\section{Study Designs}

- Randomized controlled trials

- Cohort and case-control studies

- For comparisons related to different doses, a preliminary search indicated that there are few head-to-head studies directly comparing different doses; therefore, we also included placebo-controlled studies that evaluated single doses for the purpose of potentially informing indirect comparisons related to dosing.

- For Key Question 4, we included uncontrolled longitudinal studies of patients who were successfully treated for opioid overdose with naloxone in the field and not transported to a health care facility (protocol modification due to no controlled studies being available).

\section{Study Selection and Data Extraction}

Abstracts were reviewed by two investigators to identify studies for full-text review. Two investigators then independently reviewed all full-text articles for final inclusion. Inclusion was restricted to English-language articles. Discrepancies were resolved by discussion and consensus.

For each study that was determined to meet inclusion criteria, a single investigator abstracted information on study design, year, setting, country, sample size, eligibility criteria, population and clinical characteristics, intervention characteristics (route of administration, dose/concentration, time to initial and repeat dosing, training/background of personnel administering drug), source of funding, and results relevant to each Key Question. All data abstractions were reviewed by a second investigator for accuracy. 


\section{Risk of Bias Assessment of Individual Studies}

We assessed risk of bias of included studies using predefined criteria. Two investigators independently assessed risk of bias. Disagreements were resolved by consensus. Our approach for assessing risk of bias is based on the Methods Guide for Effectiveness and Comparative Effectiveness Reviews. ${ }^{34}$ We adapted criteria for assessing risk of bias from the U.S. Preventive Services Task Force. ${ }^{35}$ For RCTs, risk of bias assessment criteria included randomization and allocation concealment methods, comparability of groups at baseline, blinding, attrition, use of intention-to-treat analysis, and prespecification of outcomes. For cohort studies, assessment criteria were based on patient selection methods; comparability of groups at baseline; methods used to ascertain exposures, confounders, and outcomes; blinding of outcomes assessors; attrition and missing data; and statistical analysis of potential confounders. For uncontrolled longitudinal studies, we used the same criteria as for cohort studies, but did not assess comparability of groups at baseline or statistical adjustment for confounders. Studies were rated as "low risk of bias," "medium risk of bias," or "high risk of bias" based on the presence and seriousness of methodological shortcomings; uncontrolled studies were rated high risk of bias since they can only address the comparative effectiveness questions addressed in this review indirectly.

\section{Assessing Research Applicability}

Factors important for understanding the applicability of studies were recorded, such as population characteristics (e.g., age, type and dose of opioid involved in overdose, or involvement of other drugs or substances), setting (United States vs. other country, out-ofhospital vs. ED administration of naloxone), and type and level of training of people administering naloxone were recorded and assessed in subgroup and sensitivity analyses to the extent possible. ${ }^{36}$ We also recorded the funding source for studies.

\section{Data Synthesis}

We constructed evidence tables with study characteristics, results, and risk of bias ratings for all included studies, and summary tables to highlight the main findings. Given the small number of studies for each Key Question and clinical and methodological heterogeneity among the studies, we determined that meta-analysis was not indicated. Rather, we synthesized studies qualitatively.

\section{Strength of the Body of Evidence}

We graded the strength of evidence for each Key Question and comparison for prioritized clinical outcomes (mortality, time to reversal of symptoms, recurrence of overdose symptoms, respiratory or cardiac arrest, rates/severity of drug withdrawal, and combativeness) by using the approach described in the AHRQ Methods Guide. ${ }^{34}$ One investigator performed the initial strength of evidence assessment and discussed with the entire team to reach consensus. 


\section{Results}

\section{Results of Literature Searches}

The search and selection of articles are summarized in the literature flow diagram (Figure B). Database searches resulted in 1,934 potentially relevant articles. After dual review of abstracts and titles, 202 articles were selected for full-text dual review, and 13 studies were determined to meet inclusion criteria and were included in this review.

Figure B. Literature flow diagram

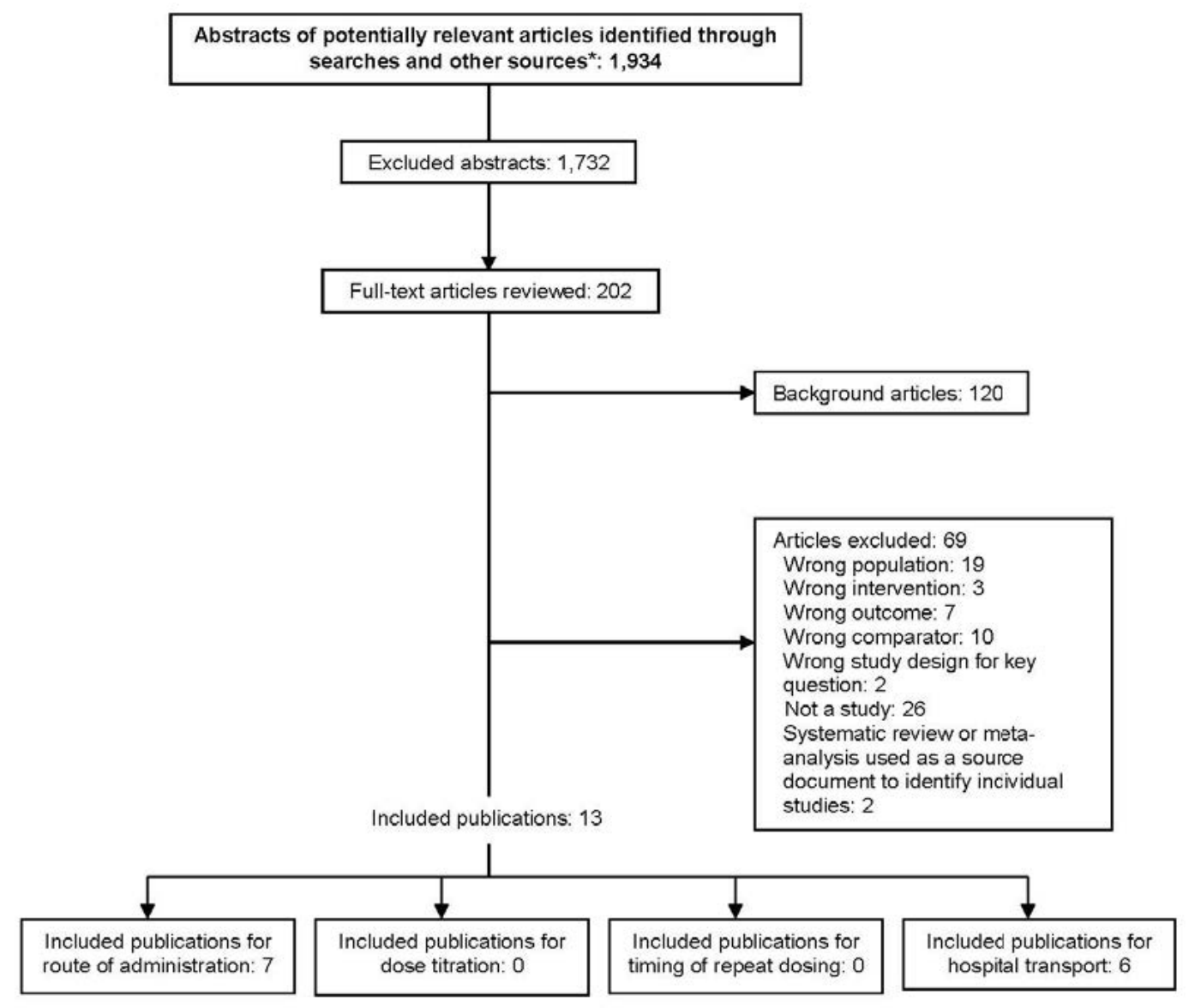

*Other sources include prior reports, references lists, referrals from experts, and grey literature

Key Question 1: For patients with confirmed or suspected opioid overdose, what are the comparative benefits and harms of out-of-hospital administration of naloxone by EMS personnel using intravenous, intramuscular, subcutaneous, and intranasal routes of administration?

We identified three RCTs ( $n=100$ to 182$)^{37-39}$ and four cohort studies ( $n=93$ to 609$)^{40-43}$ that compared different routes of naloxone administration.

- IN versus IM naloxone:

0 One trial found no difference between IN naloxone (2 mg, administered as a $2 \mathrm{mg} / 1$ $\mathrm{mL}$ formulation) versus IM naloxone (2 mg) in the likelihood of adequate response 
within 10 minutes (72\% vs. 78\%, adjusted odds ratio [OR] 0.7, 95\% confidence interval [CI] 0.3 to 1.5 ), mean response time (8.0 vs. 7.9 minutes), or agitation/violence (6.0\% vs. $7.9 \%$, relative risk [RR] $0.77,95 \%$ CI 0.25 to 2.3 ). IN naloxone was associated with increased likelihood of rescue naloxone use (18\% vs. $4.5 \%$, adjusted OR 4.8, 95\% CI 1.4 to 16 ).

0 Another trial found lower concentration IN naloxone (2 mg administered as a $2 \mathrm{mg} / 5$ $\mathrm{mL}$ formulation) associated with lower likelihood of spontaneous respirations within 8 minutes ( $63 \%$ vs. $82 \%$, OR $0.38,95 \%$ CI 0.18 to 0.81 ), higher likelihood of rescue naloxone use ( $26 \%$ vs. $13 \%$, OR 2.4, 95\% CI 1.0 to 5.7 ), longer time to respirations $>10$ /minutes ( 8 vs. 6 minutes, $p=0.006$ ), and trend towards decreased likelihood of Glasgow Coma Scale score $>11$ at 8 minutes ( $57 \%$ vs. $72 \%$, OR 0.52, 95\% CI 0.27 to 1.0) than IM naloxone (2 mg). IN naloxone was associated with decreased risk of agitation and/or irritation (2.4\% vs. $14 \%$, RR 0.19 , 95\% CI 0.04 to 0.83 ).

o The strength of evidence (SOE) for this comparison was low, due to moderate study limitations and inconsistency.

- IN versus IV naloxone:

0 One trial conducted in an Iranian ED setting found that IN naloxone ( $0.4 \mathrm{mg}$, administered as a $0.4 \mathrm{mg} / 2 \mathrm{~mL}$ formulation) was associated with a greater likelihood than IV naloxone $(0.4 \mathrm{mg}$ ) of an adequate response (defined as level of consciousness following naloxone of lethargic or conscious, $100 \%$ vs. $60 \%$, RR 1.7, 95\% CI 1.3 to 2.1 ) and lower likelihood of agitation than IV naloxone ( $0 \%$ vs. $24 \%$, RR $0.04,95 \%$ CI 0.002 to 0.66 ). The SOE was insufficient, due to moderate study limitations, inability to assess consistency, and indirectness (poor applicability to U.S. field settings due to high proportion of overdoses related to use of opium and ED setting).

o Two cohort studies reported few clear differences between IN and IV naloxone, but had serious methodological shortcomings, including failure to adjust for confounders (SOE: insufficient).

Key Question 1a: For patients with confirmed or suspected opioid overdose who receive naloxone in the out-of-hospital setting, what are the comparative benefits and harms of administration of different intravenous, intramuscular, subcutaneous, and intranasal doses of naloxone?

- No study compared different doses of naloxone administered via the same route; there was too much clinical heterogeneity to determine effects of dose from indirect comparisons (SOE: insufficient).

Key Question 2: For patients with confirmed or suspected opioid overdose in out-of-hospital settings, what are the comparative benefits and harms of titration of naloxone administered by EMS personnel until the patient resumes sufficient spontaneous respiratory effort versus until the patient regains consciousness?

- No study compared benefits and harms of titration of naloxone until the patient resumes sufficient spontaneous respiratory effort versus until the patient regains consciousness (SOE: insufficient). 
Key Question 3: For patients with confirmed or suspected opioid overdose in out-of-hospital settings treated with multiple doses of naloxone (including patients who do not improve after an initial dose of intranasal naloxone), what are the effects on benefits and harms of differences in timing of repeat dosing?

- No study compared benefits and harms of differences in timing of repeat dosing of naloxone (SOE: insufficient).

Key Question 4. For patients with confirmed or suspected opioid overdose in out-of-hospital settings who regain sufficient spontaneous respiratory effort and are alert and oriented after naloxone administration by EMS personnel, what are the benefits and harms of transporting patients to a health care facility versus nontransport?

No study compared outcomes among patients with confirmed or suspected opioid overdose who responded to naloxone administration and were transported to a health care facility versus not transported. Six studies ( $\mathrm{n}=84$ to 2241) reported on outcomes in patients who received naloxone for opioid overdose and were not transported to a health care facility. ${ }^{44-49}$

- Among patients who were successfully treated for opioid overdose by naloxone in the field and not transported to a hospital, uncontrolled studies reported rates of deaths within 0 to 2 days were 0 percent in three studies (total $\mathrm{N}=1867$ ), 0.6 percent in one study and 0.49 percent $(1 / 205)$ in another study; one study reported one case of a life-threatening adverse event (1.25\% [1/84]) (SOE: insufficient).

- No study evaluated outcomes such as linkage to treatment for opioid use disorder or subsequent repeat opioid overdose episodes.

\section{Discussion}

\section{Key Findings and Strength of Evidence}

While field administration of naloxone is generally effective in reversing opioid overdose, evidence to inform optimal management of suspected opioid overdose with naloxone by EMS personnel in terms of forms of administration or dosage is limited. We identified no previously published systematic review addressing the Key Questions in our report. Our findings are generally consistent with the conclusions of a recent FDA meeting that focused on naloxone dosing for devices intended for use by laypeople. ${ }^{26}$ The committee convened by the FDA generally found a lack of evidence to determine optimal dosing of naloxone. ${ }^{50}$

Three RCTs directly compared different routes of naloxone administration, but all had methodological shortcomings, including use of unblinded design. ${ }^{37-39}$ Among the three trials, two compared IN versus IM naloxone ${ }^{37,38}$ and one trial evaluated IN versus IV naloxone. ${ }^{39}$ No trial evaluated the recently FDA-approved naloxone auto-injector for IM administration or highly concentrated ( $4 \mathrm{mg} / 0.1 \mathrm{~mL}$ or $2 \mathrm{mg} / 0.1 \mathrm{~mL}$ ) IN formulations of naloxone.

For IN compared with IM naloxone, results suggest that a higher concentration formulation of IN naloxone ( $2 \mathrm{mg} / 1 \mathrm{~mL}$ ) is similar in efficacy to IM naloxone (SOE: low) ${ }^{38}$ Although another trial found the same dose of IN naloxone using a lower concentration formulation (2 $\mathrm{mg} / 5 \mathrm{~mL}$ ) to be less effective than IM naloxone, ${ }^{37}$ these findings are of limited applicability to 
the United States, where off-label IN naloxone is typically given at a concentration of $2 \mathrm{mg} / 2 \mathrm{~mL}$ and FDA-approved concentrations are 4 or $2 \mathrm{mg} / 0.1 \mathrm{~mL}$. Evidence regarding other route of administration comparisons is even more limited, with one trial of IN compared with IV naloxone conducted in an Iranian ED setting (SOE: insufficient). Observational studies were of very limited usefulness for informing route of administration comparisons, due to serious methodological shortcomings, including failure to adjust for potential confounders. ${ }^{40-43}$ In addition, the route of administration comparisons varied across the studies.

Evidence was insufficient to determine how comparative benefits and harms of different routes of naloxone administration differed according to demographics or clinical factors, such as the type and dose of opioid involved in overdose (including whether fentanyl or a fentanyl analogue was involved), presence of other drugs or substances, estimated time since overdose, concomitant psychiatric comorbidities, or prior overdose episodes. There was also insufficient evidence to determine how the type or training of EMS personnel administering naloxone impacted comparisons involving different routes of administration or doses of naloxone. There was insufficient evidence to determine the optimal dose of naloxone by route of administration. No study directly compared different doses of naloxone administered via the same route. It was not possible to determine effects of dose via indirect comparisons based on the studies of route of administration comparisons, given the small number of studies and differences in factors other than dose.

Evidence to determine effects of hospital transport versus nontransport following successful treatment of opioid overdose was too limited to reach reliable conclusions. No study compared outcomes in patients transported to a hospital versus those not transported following successful reversal of opioid overdose with naloxone. Although six studies reported low rates (0 to 1.2\%) of death or serious adverse events among patients who received naloxone for opioid overdose and refused transport to a health care facility, ${ }^{44-49}$ there was no comparison group of patients who were transported, which makes findings difficult to interpret, as patients who refuse transport or are assessed as not requiring transport are likely to differ substantially from patients who are transported.

No study compared titration of naloxone administered by EMS personnel until the patient resumes sufficient spontaneous respiratory effort versus until the patient regains consciousness or differences in timing of repeat naloxone dosing.

\section{Applicability}

Several factors limited the applicability of our findings. A key applicability limitation is that all studies meeting inclusion criteria evaluated older formulations of naloxone. No study evaluated the FDA-approved naloxone auto-injector for IM administration, at either a dose of 0.4 mg or the very recently approved $2 \mathrm{mg}$ dose. Similarly, no study evaluated the recently FDAapproved formulations of highly-concentrated IN naloxone. One trial evaluated IN naloxone at a concentration lower $(2 \mathrm{mg} / 5 \mathrm{~mL})$ than used off-label in the United States $(2 \mathrm{mg} / 2 \mathrm{~mL})^{37}$ and another trial evaluated IN naloxone at a concentration $(2 \mathrm{mg} / 1 \mathrm{~mL})$ that appears to have been formulated specifically for that study, and is not available in any product otherwise. ${ }^{38}$ Studies indicate very high usability rates (>90\%) with the auto-injector and FDA-approved IN naloxone, even without prior training, compared with older/off-label devices. ${ }^{51,52}$

The settings of some studies may also limit applicability to use of naloxone in U.S. field settings by EMS personnel. All of the RCTs that compared naloxone routes of administration 
were conducted in non-U.S. settings (Australia and Iran). In the Iranian trial, a high proportion of opioid overdoses were related to ingestion of opium; it was also conducted in an ED setting. ${ }^{39}$

Applicability was also limited by the populations evaluated in the studies. In almost all studies, characteristics of the opioid overdose were not reported. In addition, almost all studies were conducted before the recent increase in availability of high potency synthetic opioids. In studies regarding patients who received naloxone for opioid overdose who were not transported to a health care facility, details regarding the characteristics of patients were limited. This poses a challenge for interpreting the results of these studies, because patients who refuse transport are likely to differ substantially from patients who are transported. One study reported that 100 percent of patients who were not transported to an ED had a Glasgow Coma Scale score of 14 or 15, compared with about 50 percent of patients who were transported, but the study did not compare outcomes in patients transported versus those not transported. ${ }^{44}$

\section{Research Recommendations}

Additional research is urgently needed to optimize administration of naloxone by EMS personnel. Randomized controlled trials in U.S. field settings that compare the FDA-approved IN formulations of naloxone versus IM auto-injectors (0.4 or $2 \mathrm{mg}$ doses), compare effects of the FDA-approved formulations versus non-FDA approved versions, and compare different doses for a given route of administration (e.g., 0.4 vs. 2 mg doses of the IM auto-injector) are needed. Randomized controlled trials could pose ethical and logistical challenges in field settings, such as requiring an exception to informed consent or the need to obtain consent prior to an overdose event occurring, which would pose a challenge in identifying and engaging at-risk populations.

In addition to studies of naloxone administration by EMS personnel, studies of naloxone administration by non-EMS first responders and laypersons with limited medical training could also be informative for understanding optimal use of naloxone by Basic Life Support personnel. Ideally, studies would include (to the extent possible) information regarding the opioids involved in the overdoses and other patient factors. Studies should evaluate benefits as well as important harms, including withdrawal, agitation, aspiration, and injury.

Future research could leverage existing EMS registries with naloxone administration data, which are available from a number of local and state agencies. In addition, the National Highway Traffic Safety Administration (NHTSA)-funded National Emergency Medical Services Information System (NEMSIS) contains data from EMS agencies across the United States. ${ }^{53}$ Ideally, registry studies should include information about the dose, formulation, and route of administration of naloxone; opioid involved in exposure; training of EMS personnel administering naloxone; clinical response to initial and repeat dosing; protocol for initial and repeat naloxone dosing; and clinical outcomes, including response rates using predefined criteria, risk for recurrence of opioid overdose symptoms, and adverse outcomes. Importantly, observational studies should be designed to reduce risk of confounding and bias, including statistical adjustment.

Research is also needed to determine optimal timing and strength of dose(s) of repeat dosing as well as whether to dose until fully conscious or until patients have adequate respirations (e.g., in situations in which adequate ventilatory support is not available). For studies addressing either of these questions, the protocols used for naloxone dosing will need to be clearly defined, including indications for additional "rescue" dosing. Registry and pilot studies would be helpful for informing appropriate naloxone dosing protocols, to aid in the design of future clinical trials. 
For comparing effects of nontransport following successful treatment of opioid overdose with naloxone, RCTs may not be logistically or ethically feasible. However, comparative observational studies would better inform this question than the noncomparative studies currently available. For example, studies could identify patients who are not transported to a hospital and match them with patients who are transported, based on factors such as age, sex, suspected opioid involved in overdose, response to naloxone (e.g., based on Glasgow Coma Scale score), other substances and drugs involved in overdose, or other factors. Studies should supplement use of medical examiner and hospital records to identify outcomes with formal followup assessments, and evaluate outcomes such as linkage to treatment for opioid use disorder and risk of future overdose episodes, in addition to serious adverse outcomes such as death.

\section{Conclusions}

Low-strength evidence suggests that IN naloxone at a dose of $2 \mathrm{mg}$ and concentration of 2 $\mathrm{mg} / 1 \mathrm{~mL}$ is similar in efficacy to IM naloxone at a dose of $2 \mathrm{mg}$, with no difference in adverse events. Research is needed on the comparative effectiveness of the FDA-approved naloxone auto-injectors and highly concentrated IN naloxone formulations, different doses, and dosing strategies. Uncontrolled studies suggest that nontransport of patients following successful naloxone reversal of overdose might be associated with a low rate of serious harms, but patients were probably at low risk for such events, and there is insufficient evidence to determine risk of adverse effects for transported versus nontransported patients after opioid reversal in the field setting. 


\section{References}

1. Macrae J, Hyde P, Slavitt A. HHS launches multi-pronged effort to combat opioid abuse Washington, DC: U.S. Department of Health \& Human Services; July 27,

2015. http://www.hhs.gov/blog/2015/07/27/ hhs-launches-multi-pronged-effort-combatopioid-abuse.html. Accessed August 29, 2016.

2. Centers for Disease Control and Prevention. Vital signs: overdoses of prescription opioid pain relievers---United States, 1999--2008. FIGURE 2. Rates of opioid pain reliever (OPR) overdose death, OPR treatment admissions, and kilograms of OPR sold --United States, 1999--2010. MMWR Morb Mortal Wkly Rep. 2011 Nov 4;60(43):148792. PMID: 22048730.

3. Rudd RA, Seth P, David F, et al. Increases in drug and opioid overdose deaths--United States, 2010-2015. MMWR Morb Mortal Wkly Rep. 2016;65(50-51):1445-52.

4. National Center for Health Statistics. Drugpoisoning deaths involving opioid analgesics: United States, 1999-2011. NCHS Data Brief 166. Atlanta, GA: Centers for Disease Control and Prevention; September 2014. https://www.cdc.gov/nchs/products/da tabriefs/db166.htm. Accessed May 25, 2016.

5. Schumann H, Erickson T, Thompson TM, et al. Fentanyl epidemic in Chicago, Illinois and surrounding Cook County. Clin Toxicol. 2008 Jul;46(6):501-6. doi: 10.1080/15563650701877374. PMID: 18584361 .

6. Frank RG, Pollack HA. Addressing the fentanyl threat to public health. N Engl J Med. 2017 Feb 16;376(7):605-7. doi: 10.1056/NEJMp1615145. PMID: 28199808.

7. Tomassoni AJ, Hawk KF, Jubanyik K, et al. Multiple fentanyl overdoses - New Haven, Connecticut, June 23, 2016. MMWR Morb Mortal Wkly Rep. 2017 Feb 03;66(4):10711. doi: 10.15585/mm6604a4. PMID: 28151928.
8. Somerville NJ, O'Donnell J, Gladden RM, et al. Characteristics of fentanyl overdose Massachusetts, 2014-2016. MMWR Morb Mortal Wkly Rep. 2017 Apr 14;66(14):3826. doi: 10.15585/mmwr.mm6614a2. PMID: 28406883.

9. Sutter ME, Gerona RR, Davis MT, et al. Fatal fentanyl: One pill can kill. Acad Emerg Med. 2016 Jun 20doi: 10.1111/acem.13034. PMID: 27322591.

10. Strang J, McDonald R, Alqurshi A, et al. Naloxone without the needle - systematic review of candidate routes for non-injectable naloxone for opioid overdose reversal. Drug Alcohol Depend. 2016 Jun 1;163:16-23. doi: 10.1016/j.drugalcdep.2016.02.042. PMID: 26996745.

11. FDA approves new hand-held auto-injector to reverse opioid overdose. FDA news release, April 3, 2014. Silver Spring, MD: U.S. Food and Drug Administration; 2014. http://www.fda.gov/NewsEvents/New sroom/PressAnnouncements/ucm391465.ht m. Accessed February 15, 2017.

12. FDA moves quickly to approve easy-to-use nasal spray to treat opioid overdose. FDA news release, Nov. 18, 2015. Silver Spring, MD: U.S. Food and Drug Administration; 2015. http://www.fda.gov/NewsEvents/New sroom/PressAnnouncements/ucm473505.ht m. Accessed August 29, 2016.

13. Kim D, Irwin KS, Khoshnood K. Expanded access to naloxone: options for critical response to the epidemic of opioid overdose mortality. Am J Public Health. 2009 Mar;99(3):402-7. doi: 10.2105/AJPH.2008.136937. PMID: 19150908.

14. Boyer EW. Management of opioid analgesic overdose. N Engl J Med. 2012 Jul 12;367(2):146-55. doi: 10.1056/NEJMra1202561. PMID: 22784117.

15. Wermeling DP. Review of naloxone safety for opioid overdose: practical considerations for new technology and expanded public access. Ther Adv Drug Saf. 2015

Feb;6(1):20-31. doi:

10.1177/2042098614564776. PMID: 25642320. 
16. Buajordet I, Naess AC, Jacobsen D, et al. Adverse events after naloxone treatment of episodes of suspected acute opioid overdose. Eur J Emerg Med. 2004 Feb;11(1):19-23. PMID: 15167188.

17. EMS1. 5 things EMS providers need to know about opioid overdose and respiratory compromise.

2016. http://www.ems1.com/emsproducts/Capnography/articles/47753048-5things-EMS-providers-need-to-know-aboutopioid-overdose-and-respiratorycompromise/. Accessed October 7, 2016.

18. Alcorn T. America embraces treatment for opioid drug overdose. Lancet. 2014 Jun 7;383(9933):1957-8. PMID: 24936622.

19. Fala L, Welz JA. New perspectives in the treatment of opioid-induced respiratory depression. Am Health Drug Benefits. 2015 Oct;8(6 Suppl 3):S51-63. PMID: 26629261.

20. Alexander JL, Burton JH, Bradshaw JR, et al. Suspected opioid-related emergency medical services encounters in a rural state, 1997-2002. Prehosp Emerg Care. 2004 OctDec;8(4):427-30. PMID: 15626007.

21. National EMS Information System (NEMSIS). Create a report (NEMSIS Cube). 2017. http://nemsis.org/reportingTools/repor ts/nationalReports/createAReport.html.

Accessed Februrary 15, 2017.

22. National Highway Traffic Safety Administration. The national EMS scope of practice model. DOT HS 810657. Washington, DC: U.S. Department of Transportation; February 2007. http://www.ems.gov/education/EMSS cope.pdf Accessed August 29, 2016.

23. Lavonas EJ, Drennan IR, Gabrielli A, et al. Part 10: Special circumstances of resuscitation: 2015 American Heart Association guidelines update for cardiopulmonary resuscitation and emergency cardiovascular care. Circulation. 2015 Nov 3;132(18 Suppl 2):S501-18. doi: 10.1161/CIR.0000000000000264. PMID: 26472998.
24. National Association of State EMS Officials. National model EMS clinical guidelines.

2014. https://www.nasemso.org/Projects/Mo delEMSClinicalGuidelines/documents/Natio nal-Model-EMS-Clinical-Guidelines230ct2014.pdf. Accessed August 29, 2016.

25. World Health Organization (WHO). Community management of opioid overdose. Geneva, Switzerland; 2014. http://www.who.int/substance_abuse/ publications/management_opioid_overdose/ en/.

26. U.S. Food and Drug Administration. 2016

Meeting Materials, Anesthetic and Analgesic Drug Products Advisory Committee.

2016. http://www.fda.gov/AdvisoryCommitt ees/CommitteesMeetingMaterials/Drugs/An estheticAndAnalgesicDrugProductsAdvisor yCommittee/ucm486848.htm.

27. Adapt Pharma Operations Limited. FDA Advisory Committee on the most appropriate dose or doses of naloxone to reverse the effects of life-threatening opiod overdose in the community settings. 2016. http://www.fda.gov/downloads/Advis oryCommittees/CommitteesMeetingMaterial s/Drugs/AnestheticAndAnalgesicDrugProdu ctsAdvisoryCommittee/UCM522688.pdf.

28. Amphastar Pharmaceuticals. FDA Advisory Committee Briefing Materials. Intranasal naloxone intended for use in the community. Joint meeting of the anesthetic and analgesic drug products advisory committee and the drug safety and risk management advisory committee.

2016. http://www.fda.gov/downloads/Advis oryCommittees/CommitteesMeetingMaterial s/Drugs/AnestheticAndAnalgesicDrugProdu ctsAdvisoryCommittee/UCM522689.pdf.

29. Amphastar Pharmaceuticals. Naloxone meeting: Joint meeting of the anesthetic and alagesic drug products advisory committee and the drug safety and risk management advisory committee.

2016. http://www.fda.gov/downloads/Advis oryCommittees/CommitteesMeetingMaterial s/Drugs/AnestheticAndAnalgesicDrugProdu ctsAdvisoryCommittee/UCM524334.pdf. 
30. Insys Development Company Inc. Naloxone for treatment of opioide overdose 2016. http://www.fda.gov/downloads/Advis oryCommittees/CommitteesMeetingMaterial s/Drugs/AnestheticAndAnalgesicDrugProdu ctsAdvisoryCommittee/UCM522690.pdf

Accessed December 19, 2016.

31. Kaleo Inc. Joint meeting of the anesthetic and analgesic drug products and the drug safety and risk management advisory committees.

2016. http://www.fda.gov/downloads/Advis oryCommittees/CommitteesMeetingMaterial s/Drugs/AnestheticAndAnalgesicDrugProdu ctsAdvisoryCommittee/UCM522691.pdf.

32. U.S. Food and Drug Administration. Joint meeting of anesthetic and analgesic drug products advisory committee and drug safety and risk management advisory committee.

2016. http://www.fda.gov/downloads/Advis oryCommittees/CommitteesMeetingMaterial s/Drugs/AnestheticAndAnalgesicDrugProdu ctsAdvisoryCommittee/UCM522687.pdf.

33. McDonagh M, Peterson K, Raina $\mathrm{P}$, et al. Avoiding bias in selecting studies. Methods guide for comparative effectiveness reviews. (Prepared by the Oregon Health \& Science University, McMaster University, and Southern California Evidence-based Practice Centers under Contract No. 290-200710057-I.) AHRQ Publication No. 13EHC045-EF. Rockville, MD: Agency for Healthcare Research and Quality; 2013. www.effectivehealthcare.ahrq.gov/reports/fi nal.cfm. Accessed August 29, 2016.

34. Agency for Healthcare Research and Quality. Methods guide for effectiveness and comparative effectiveness reviews. 2014. www.effectivehealthcare.ahrq.gov. Accessed January 23, 2017.

35. U.S. Preventive Services Task Force. U.S. Preventive Services Task Force procedure manual.

2008. http://www.uspreventiveservicestaskf orce.org/uspstf08/methods/procmanual.htm.

36. Atkins D, Chang SM, Gartlehner G, et al. Assessing applicability when comparing medical interventions: AHRQ and the Effective Health Care Program. J Clin Epidemiol. 2011 Nov;64(11):1198-207. doi: 10.1016/j.jclinepi.2010.11.021. PMID: 21463926.
37. Kelly AM, Kerr D, Dietze P, et al. Randomised trial of intranasal versus intramuscular naloxone in prehospital treatment for suspected opioid overdose. Med J Aust. 2005 Jan 3;182(1):24-7. PMID: 15651944.

38. Kerr D, Kelly AM, Dietze P, et al. Randomized controlled trial comparing the effectiveness and safety of intranasal and intramuscular naloxone for the treatment of suspected heroin overdose. Addiction. 2009 Dec;104(12):2067-74. doi: 10.1111/j.13600443.2009.02724.x. PMID: 19922572.

39. Sabzghabaee AM, Eizadi-Mood N, Yaraghi A, et al. Naloxone therapy in opioid overdose patients: intranasal or intravenous? a randomized clinical trial. Arch Med Sci. 2014 May 12;10(2):309-14. doi: 10.5114/aoms.2014.42584. PMID: 24904666.

40. Merlin MA, Saybolt M, Kapitanyan R, et al. Intranasal naloxone delivery is an alternative to intravenous naloxone for opioid overdoses. Am J Emerg Med. 2010 Mar;28(3):296-303. doi: 10.1016/j.ajem.2008.12.009. PMID: 20223386.

41. Robertson TM, Hendey GW, Stroh G, et al. Intranasal naloxone is a viable alternative to intravenous naloxone for prehospital narcotic overdose. Prehosp Emerg Care. 2009 Oct-Dec;13(4):512-5. doi: 10.1080/10903120903144866. PMID: 19731165.

42. Sporer KA, Firestone J, Isaacs SM. Out-ofhospital treatment of opioid overdoses in an urban setting. Acad Emerg Med. 1996 Jul;3(7):660-7. PMID: 8816181.

43. Wanger K, Brough L, Macmillan I, et al. Intravenous vs subcutaneous naloxone for out-of-hospital management of presumed opioid overdose. Acad Emerg Med. 1998 Apr;5(4):293-9. PMID: 9562190.

44. Boyd JJ, Kuisma MJ, Alaspaa AO, et al. Recurrent opioid toxicity after pre-hospital care of presumed heroin overdose patients. Acta Anaesthesiol Scand. 2006 Nov;50(10):1266-70. PMID: 17067327. 
45. Levine M, Sanko S, Eckstein M. Assessing the risk of prehospital administration of naloxone with subsequent refusal of care. Prehosp Emerg Care. 2016;20(5):566-9. PMID: 27018626.

46. Vilke GM, Buchanan J, Dunford JV, et al. Are heroin overdose deaths related to patient release after prehospital treatment with naloxone? Prehosp Emerg Care. 1999 JulSep;3(3):183-6. PMID: 10424852.

47. Vilke GM, Sloane C, Smith AM, et al. Assessment for deaths in out-of-hospital heroin overdose patients treated with naloxone who refuse transport. Acad Emerg Med. 2003 Aug;10(8):893-6. PMID: 12896894.

48. Wampler DA, Molina DK, McManus J, et al. No deaths associated with patient refusal of transport after naloxone-reversed opioid overdose. Prehosp Emerg Care. 2011 JulSep;15(3):320-4. doi: 10.3109/10903127.2011.569854. PMID: 21612385.

49. Rudolph SS, Jehu G, Nielsen SL, et al. Prehospital treatment of opioid overdose in Copenhagen--is it safe to discharge onscene? Resuscitation. 2011

Nov;82(11):1414-8. doi:

10.1016/j.resuscitation.2011.06.027. PMID: 21745532.

50. Fiore K. FDA splits on naloxone dose. 2016. http://www.medpagetoday.com/Neuro logy/GeneralNeurology/60632.

51. Krieter P, Chiang N, Gyaw S, et al. Pharmacokinetic properties and human use characteristics of an FDA-approved intranasal naloxone product for the treatment of opioid overdose. J Clin Pharmacol. 2016 Oct;56(10):1243-53. doi: 10.1002/jcph.759. PMID: 27145977.

52. Edwards ET, Edwards ES, Davis E, et al. Comparative usability study of a novel autoinjector and an intranasal system for naloxone delivery. Pain Ther. 2015 Jun;4(1):89-105. doi: 10.1007/s40122-0150035-9. PMID: 25910473.

53. National EMS Information System (NEMSIS). The project. 2005. http://nemsis.org/theProject/index.htm l. Accessed February 15, 2017. 


\section{Introduction}

\section{Background}

\section{Nature and Burden of Opioid Overdose}

Addiction and overdoses associated with prescription and illicit opioids have been characterized by the U.S. Department of Health and Human Services as a national crisis. ${ }^{1}$ Since 2000, the rate of overdose deaths involving opioids has increased four-fold. ${ }^{2,3}$ Drug overdose deaths are now the leading cause of injury-related death in the United States. ${ }^{4}$ Overdose deaths due to opioids occur as a result of their central nervous system effects, which cause respiratory depression that can progress to cardiac arrest if untreated. In 2015, the number of drug overdose deaths involving prescription or illicit opioids exceeded 33,000, the highest number on record. ${ }^{3}$ The strongest risk factor for an opioid overdose death is a prior overdose event. Other risk factors include concomitant use of other medications and substances with central nervous system depressant effects, recent abstinence (due to decreased tolerance to opioids upon re-exposure), higher doses of opioids, obtaining opioids from multiple providers or pharmacies, presence of comorbid conditions such as sleep apnea or other respiratory disease, and genetic predisposition to the respiratory depressant effects of opioids. ${ }^{5-10}$ Of recent concern is whether dosing guidelines are sufficient for reversing overdose related to highly potent synthetic opioids (e.g. fentanyl and fentanyl analogues). ${ }^{3,11-15}$ In addition, although overdoses with opioids often involve other medications and substances with respiratory depressant effects, naloxone has minimal benefit for other types of overdoses. Children in households with an adult who is prescribed opioids are also at risk for overdose. ${ }^{16,17}$ Opioid overdoses can have serious adverse health consequences (e.g., hypoxic brain injury, aspiration, seizure), even when not fatal. Although difficult to measure, the ratio of nonfatal to fatal overdoses has been estimated to range from $7.5: 1$ to $30: 1 .^{18,19}$

\section{Field Treatment of Suspected Opioid Overdose With Naloxone}

Naloxone is an opioid antagonist that was first approved in 1971 to rapidly counteract the central nervous system (CNS) and respiratory depressant effects of opioids, potentially preventing fatal overdose. ${ }^{20}$ Naloxone can be administered by the intravenous (IV), intramuscular (IM), subcutaneous (SC), intranasal (IN), endotracheal (ET), nebulized/inhalational, buccal, or sublingual routes. ${ }^{21}$ The U.S. Food and Drug Administration (FDA) approved a handheld naloxone IM or SC auto-injector in $2014^{22}$ and a new IN formulation and delivery device in $2015 ;{ }^{23}$ both administer a consistent preset dose and were designed for ease of use even by individuals with limited or no health care training. With IN administration using a preloaded device, there is no risk of needle stick injury. In addition to administration by clinical personnel, naloxone also is associated with decreased risk of opioid overdose when distributed in community-based programs. ${ }^{24-26}$ Naloxone has been shown to be effective for reversal of opioid overdose across various routes of administration and doses. ${ }^{20,27}$

Naloxone administration may precipitate withdrawal symptoms in chronic opioid users. ${ }^{28}$ Risk of naloxone withdrawal depends on factors such as the degree of physical dependence, dose of opioid, and dose of naloxone. Reported rates of withdrawal following naloxone administration range from 7 to 46 percent. $^{29}$ While uncomfortable, withdrawal symptoms are generally not serious or life-threatening and generally short-lived. Post withdrawal agitation following 
naloxone administration may also put the person administering the naloxone at increased risk for injury. ${ }^{30,31}$ Withdrawal symptoms may be more severe with use of IV naloxone and are less likely to occur or minimized in severity by using the lowest effective doses and dose titration. $20,2820,2820,28$ The duration of action of naloxone is 20 to 90 minutes, or shorter than many opioids. 202020

Emergency medical services (EMS) personnel are often involved in management of potential opioid overdoses. When responding to opioid overdoses, early intervention is critical to prevent death and other complications of opioid overdose. ${ }^{32}$ Airway management and continuous assessment of oxygenation and ventilation, along with administration of naloxone, is the standard of care for EMS personnel treating suspected opioid overdoses. ${ }^{33}$ According to the National EMS Information System database, the number of EMS encounters for suspected opioid overdose has increased, with nearly 160,000 doses of naloxone administered by EMS personnel in $2014 .^{34}$ Regulations vary, however, with regard to whether EMS personnel with different levels of training are permitted to administer naloxone. In order of increasing level of training, EMS personnel are commonly classified as emergency medical responders (EMRs), emergency medical technicians (EMTs), intermediate/advanced EMTs, and paramedics (in most states, the intermediate EMT classification has been replaced by advanced EMTs). ${ }^{35}$ Naloxone administration is not currently within the National EMS Scope of Practice Model for EMTs and EMRs, which was last updated in $2007,{ }^{35}$ prior to the introduction of newer naloxone formulations and availability of newer evidence on the benefits of field use of naloxone. However, each state can independently amend its EMS scope of practice. A recent systematic review $^{36}$ of U.S. laws, regulations, and policies found that all jurisdictions permitted paramedics and 48 permitted intermediate/advanced EMTs to administer naloxone. Fewer jurisdictions permitted basic life support personnel to administer naloxone, which may contribute to disparities in areas in which more care is provided by EMRs and EMTs. ${ }^{37}$ One study found that among 724 patients treated in out-of-hospital settings by Basic Life Support EMS personnel and transported to a hospital, fewer than 10 percent of patients received additional doses of naloxone in the ED; there was one death. ${ }^{38}$

Although a number of recommendations, guidelines, and protocols are available to inform out-of-hospital management of opioid overdose patients, including naloxone use, guidance varies across these documents, and there are uncertainties in a number of areas. ${ }^{39-41}$ A review of naloxone dosing recommendations found that 48 percent recommended a starting dose of 0.05 mg or lower, 16 percent recommended $0.1 \mathrm{mg}$, and 36 percent recommended 0.4 or $0.5 \mathrm{mg} .{ }^{42}$ The 2015 American Heart Association (AHA) guideline update for cardiopulmonary resuscitation and emergency cardiovascular care notes that the ideal dose of naloxone is not known; an empiric starting dose of 0.04 to $0.4 \mathrm{mg}$ IV or IM is recommended to avoid provoking severe opioid withdrawal and to allow for a range of doses depending on the clinical scenario, with repeat doses or dose escalation to $2 \mathrm{mg}$ if the initial response is inadequate. ${ }^{39}$ The naloxone auto-injector approved by the FDA in 2014 administered a dose of $0.4 \mathrm{mg} .^{22}$ However, a $2 \mathrm{mg}$ auto-injector was approved in 2016 and the $0.4 \mathrm{mg}$ dose is no longer being manufactured. ${ }^{43}$ For IN naloxone, the AHA guideline notes that most studies used off-label dosing of $2 \mathrm{mg}$ in $2 \mathrm{~mL}$ of solution via an atomizer, with the dose repeated in 3 to 5 minutes if necessary. Despite clinical experience suggesting effectiveness of such off-label IN administration, potential concerns include inadequately characterized pharmacokinetics, low bioavailability, high rates of administration errors, and inadequate dosing for overdoses due to potent opioids. ${ }^{44,45}$ In 2015 , the FDA approved a reformulation of IN naloxone at a dose of $4 \mathrm{mg}$ in a highly-concentrated 
solution of $0.1 \mathrm{~mL} ;{ }^{46}$ a $2 \mathrm{mg} / 0.1 \mathrm{ml}$ formulation was approved in $2017 .{ }^{47}$ Concentrated solutions may be important for optimal IN administration due to a low rate of absorption (a high proportion of IN naloxone is swallowed and inert) with low bioavailability; a maximum of $\leq 0.5$ $\mathrm{mL}$ can be effectively delivered per nostril. Another formulation of IN naloxone did not receive FDA approval in 2015; it delivered a total of $1.8 \mathrm{mg}$ of naloxone, which was determined by the FDA to not fully meet the equivalent FDA threshold dose (0.4 mg IM naloxone). ${ }^{48}$

Therefore, there are uncertainties regarding the optimal route of naloxone administration, the optimal dose for different routes of administration, optimal dosing strategies, and appropriate training levels for EMS personnel permitted to administer naloxone. Of recent concern is whether current dosing guidelines are sufficient for reversing overdose related to highly potent synthetic opioids. ${ }^{49}$ Data suggest that the proportion of patients who receive multiple naloxone administrations by EMS personnel increased from 14 percent in 2012 to 18 percent in 2015 . $^{50}$ The increase may be related to a higher proportion of overdoses involving highly potent opioids, higher doses of opioids, or sustained-release or long-acting opioids. There is also uncertainty regarding the optimal timing of repeat naloxone dosing, as well as the optimal dose of naloxone that should be administered by various routes. Another important question is whether patients should be dosed until they achieve sufficient spontaneous respiration or until they return to full consciousness. Although dosing to full consciousness might reduce the likelihood of recurrence of overdose symptoms or complications related to decreased level of consciousness (e.g., aspiration or upper airway obstruction), a potential trade-off is increased likelihood or severity of withdrawal symptoms or refusal to be transported to a health care facility. A related question is whether mandatory hospital transport is medically necessary following successful naloxone treatment of opioid overdose, ${ }^{51}$ and whether there may be additional benefits of hospital transport in addition to those related to the current overdose episode, such as increased likelihood of linkage of care for treatment for opioid use disorder, alerting prescribers if the involved opioids were prescribed, and decreased risk of subsequent overdose.

\section{Rationale for Review}

The purpose of this systematic review is to synthesize the evidence on naloxone route of administration and dosing for suspected opioid overdose in out-of-hospital settings, and on the need for transport to a hospital following successful opioid overdose reversal with naloxone. The review is intended to inform development of evidence-based guidelines on EMS management of suspected opioid overdose with naloxone and potentially inform an update to the National EMS Scope of Practice Model regarding naloxone use across EMS training levels. Therefore, the review focuses on studies of naloxone administration by EMS personnel. Because studies of naloxone administration by non-EMS first responders and laypeople may also have some applicability for EMS administration, we also included studies of naloxone administration by non-EMS personnel.

\section{Scope and Key Questions}

The report addresses the following Key Questions. 
Key Question 1: For patients with confirmed or suspected opioid overdose, what are the comparative benefits and harms of out-of-hospital administration of naloxone by EMS personnel using intravenous, intramuscular, subcutaneous, and intranasal routes of administration?

1a. For patients with confirmed or suspected opioid overdose who receive naloxone in the out-of-hospital setting from EMS personnel, what are the comparative benefits and harms of different intravenous, intramuscular, subcutaneous, or intranasal doses of naloxone?

Key Question 2: For patients with confirmed or suspected opioid overdose in out-of-hospital settings, what are the comparative benefits and harms of titration of naloxone administered by EMS personnel until the patient resumes sufficient spontaneous respiratory effort versus until the patient regains consciousness?

Key Question 3: For patients with confirmed or suspected opioid overdose in out-of-hospital settings treated with multiple doses of naloxone (including patients who do not improve after an initial dose of intranasal naloxone), what are the effects on benefits and harms of differences in timing of repeat dosing?

Key Question 4: For patients with confirmed or suspected opioid overdose in out-of-hospital settings who regain sufficient spontaneous respiratory effort and are alert and oriented after naloxone administration by EMS personnel, what are the benefits and harms of transporting patients to a health care facility versus nontransport?

The research questions used to guide this review were initially developed by the National Highway Traffic Safety Administration (NHTSA) and revised with input from a Technical Expert Panel (TEP). The Key Questions focus on the comparative benefits and harms of alternative routes of administration, dose, and dosing strategies for naloxone administered for suspected opioid overdose in the field, and benefits and harms of transport to a health care facility versus nontransport after successful treatment of suspected opioid overdose with naloxone.

The analytic framework (Figure 1) shows the target population, interventions, and health outcomes examined; the Key Questions are numbered and indicated in the framework. We focused on use of IN, IM, and IV naloxone; these are the formulations of naloxone most commonly used for reversal of suspected opioid overdose in the field by EMS personnel. Key Question 1 addresses benefits and harms of different routes of naloxone administration, Key Question 1a addresses benefits and harms of different doses of naloxone administration, Key Question 2 addresses benefits and harms of dosing until the patient resumes sufficient spontaneous respiratory effort but is not necessarily fully conscious versus until the patient regains full consciousness, Key Question 3 addresses benefits and harms of differences in timing 
of repeat dosing, and Key Question 4 addresses benefits and harms of transporting patients following successful reversal of naloxone overdose versus nontransport. Although the focus of the review was on use of naloxone by field personnel, we also included studies of naloxone administration in out-of-hospital settings by non-EMS personnel (e.g., police or other laypeople), which could help inform optimal dosing strategies by EMS personnel with additional training.

\section{Analytic Framework}

Figure 1. Analytic framework

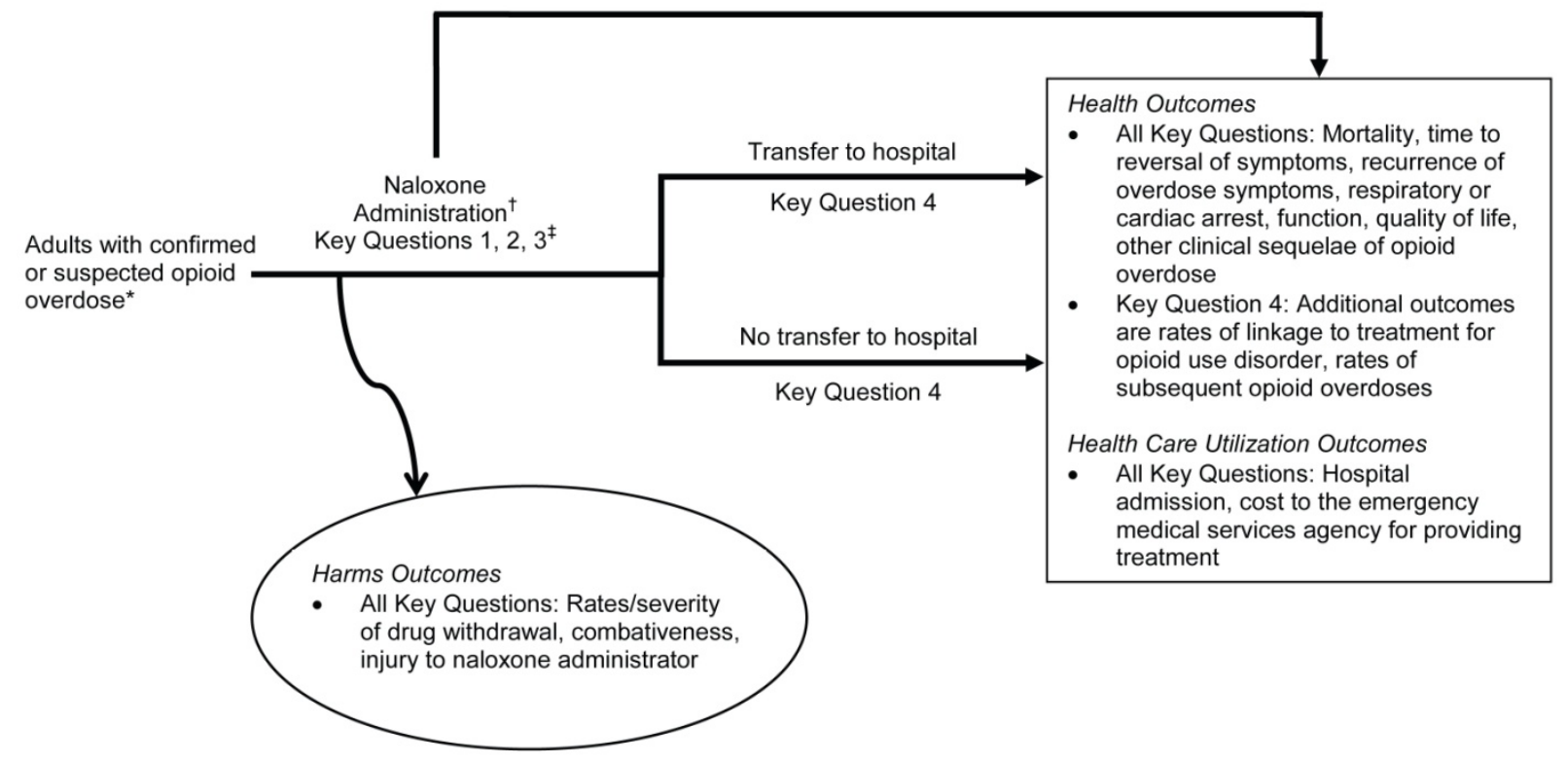

\footnotetext{
* Patients with confirmed or suspected opioid overdose who exhibit altered mental status, miosis, or respiratory distress and who are treated in the out-of-hospital setting by emergency medical services personnel

†Administration of naloxone hydrochloride via the nasal, intravenous, intramuscular, or subcutaneous injection (including the naloxone auto-injector)

‡ Key Question 1 addresses comparisons involving route of administration and dose; Key Question 2 addresses comparisons involving dose titration to varying degrees of return of consciousness (intermediate outcome)
} 


\section{Methods}

The methods for this Comparative Effectiveness Review (CER) are based on the guidance in the Agency for Healthcare Research and Quality (AHRQ) Methods Guide for Effectiveness and Comparative Effectiveness Reviews. ${ }^{52}$

\section{Topic Refinement and Review Protocol}

The initial Key Questions were provided by the National Highway Traffic Safety Administration (NHTSA). The Key Questions were further developed and the final protocol developed with additional input from NHTSA and a Technical Expert Panel (TEP) convened for this report. The TEP consisted of 10 experts in addiction medicine, pain medicine, emergency medical services (EMS), emergency medicine, toxicology, pharmacy, epidemiology of opioid overdose, opioid overdose research, and public policy. TEP members disclosed financial and other conflicts of interest prior to participation. The AHRQ Task Order Officer and the investigators reviewed the disclosures and determined that the TEP members had no conflicts of interest that precluded participation.

The final protocol was posted on the AHRQ Web site on November 30, 2016 at: https://www.effectivehealthcare.ahrq.gov/search-for-guides-reviews-andreports/?pageaction=displayproduct\&productid $=2360$. The protocol was also registered in the PROSPERO international database of prospectively registered systematic reviews (registration number CRD42016053891). Two modifications were made to the protocol after posting: we expanded the inclusion criteria for Key Question 1 to include studies performed in an emergency department (ED) setting and we expanded the inclusion criteria for Key Question 4 to include uncontrolled longitudinal studies of patients successfully treated for suspected opioid overdose in the field who were not transported to a health care facility.

\section{Literature Search Strategy}

\section{Search Strategy}

A research librarian conducted searches in Ovid MEDLINE ${ }^{\circledR}$ (1946-August Week 2 2016), PsycINFO $^{\circledR}$, the Cochrane Central Register of Controlled Trials (CCRCT), and the Cumulative Index to Nursing and Allied Health Literature (CINAHL ${ }^{\circledR}$ ). Search strategies are shown in Appendix A. We did not apply search date restrictions and updated searches were conducted through Septebmer 2017. The AHRQ Evidence-based Practice Center (EPC) Scientific Resource Center (SRC) sent email notification to relevant stakeholders about the opportunity to submit Scientific Information Packets (SIP) via the Effective Health Care (EHC) Web site for naloxone. The SIP invitations requested published or unpublished evidence relevant to the review and were assessed according to the criteria and processes described for all evidence, below.

We also hand-searched reference lists of relevant studies, searched for unpublished or ongoing studies in ClinicalTrials.gov, contacted representatives of federal agencies involved in naloxone or opioid overdose research (CDC, NIDA, and SAMHSA) for additional studies, reviewed medical and statistical reviews on the FDA's Center for Drug Evaluation and Research Web site, and reviewed materials presented at a recent FDA meeting ${ }^{53}$ on naloxone dosing. ${ }^{54-59}$ 


\section{Inclusion and Exclusion Criteria}

We developed preestablished criteria for inclusion and exclusion of studies based on the Key Questions and the populations, interventions, comparators, outcomes, timing, types of studies, and setting (PICOTS) approach, in accordance with the AHRQ EPC Methods Guide for Effectiveness and Comparative Effectiveness Reviews. ${ }^{60}$ Inclusion and exclusion criteria are described below.

Abstracts were reviewed by two investigators, and all citations deemed potentially appropriate for inclusion by at least one of the reviewers were retrieved for full-text review. Two investigators then independently reviewed all full-text articles for final inclusion. Investigators did not review, assess, or screen papers that they had authored. Inclusion was restricted to English-language articles. Discrepancies were resolved by discussion and consensus, with a third investigator to resolve disagreements if necessary.

A list of the included studies is shown in Appendix B; a list of excluded studies and primary reasons for exclusion is shown in Appendix C.

\section{Population(s):}

- Include: Patients with confirmed or suspected opioid overdose who exhibit altered mental status, miosis or respiratory depression and who are treated in the out-of-hospital setting by EMS personnel

o Also include studies of naloxone administration in out-of-hospital settings by nonEMS personnel (e.g., police, other first responders, laypeople), which may inform optimal dosing strategies in EMS personnel.

o Subpopulations: Based on age, sex, race, type of opioid involved in overdose, dose of opioid involved in overdose, presence of other drugs or substances contributing to overdose, estimated time since overdose, concomitant psychiatric comorbidities, and prior overdose episodes.

o Exclude: Patients with altered mental status or respiratory distress due solely to trauma, hypoxia or ingestion of ethanol or other nonopioid substances, and patients without signs of opioid overdose treated for chronic pain or addiction with buprenorphine/naloxone.

\section{Interventions:}

- $\quad$ For Key Questions 1-3: See Table 1 for included naloxone formulations.

Table 1. Naloxone: Dose and route of administration

\begin{tabular}{|l|l|}
\hline Included Drug & Dose and Route of Administration \\
\hline Naloxone & $\bullet \quad$ Auto-injector, intramuscular $(\mathrm{IM})$ \\
& $0 \quad 0.4 \mathrm{mg} / 0.4 \mathrm{~mL},{ }^{*} 2 \mathrm{mg} / 0.4 \mathrm{~mL}$ \\
& Nasal spray, intranasal (IN) \\
& S Single dose intranasal device: $4 \mathrm{mg} / 0.1 \mathrm{~mL}, 2 \mathrm{mg} / 0.1 \mathrm{~mL}$ \\
$0 \quad$ Improvised intranasal device: $2 \mathrm{mg} / 2 \mathrm{~mL}^{\dagger}$ & \\
& Injection, intravenous, intramuscular or subcutaneous \\
& $0 \quad 0.4 \mathrm{mg} / \mathrm{mL}, 1 \mathrm{mg} / \mathrm{mL}, 2 \mathrm{mg} / \mathrm{mL}$ \\
\hline
\end{tabular}

* Manufacturer has stopped production of $0.4 \mathrm{mg} / 0.4 \mathrm{~mL}$ IM

$\dagger$ Formulation not currently approved by the Food and Drug Administration for intranasal administration

- Potential modifiers of interventions: Based on training and background of the person administering naloxone 
- For Key Question 4: Transport to health care facility

- Exclude: Naloxone in combination with other medications (e.g., buprenorphine/ naloxone)

\section{Comparators:}

- Key Question 1: Injection (intramuscular, subcutaneous or intravenous) versus intranasal route of administration

- Key Question 1a: Comparisons of different doses of intranasal, intramuscular, and intravenous naloxone

- Key Question 2: Titration of patients until they resume spontaneous respiration but have some residual sedation/altered mental status versus dosing of patient until they resume spontaneous respiration and are awake and alert

- Key Question 3: Comparison of differences in timing of repeat dosing

- Key Question 4: Transport of patients following treatment of opioid overdose with naloxone to a health care facility versus nontransport

\section{Outcomes:}

- All Key Questions: Mortality, reversal of overdose symptoms (based on return of adequate spontaneous respirations or level of consciousness), time to reversal of symptoms, recurrence of overdose symptoms, respiratory or cardiac arrest, function, quality of life, other clinical sequelae of opioid overdose (e.g. noncardiogenic pulmonary edema); health care utilization indicators (e.g., hospital admission, cost to the EMS agency for providing treatment); and adverse effects and other harms (such as rates/severity of drug withdrawal, combativeness, injury to administrator of naloxone)

- Key Question 4: Additional outcomes are rates of linkage to treatment for opioid use disorder, rates of subsequent opioid overdoses

\section{Timing:}

- No restrictions on timing of followup

\section{Settings:}

- Include: Out-of-hospital setting, ED setting (for Key Questions 1 and 1a). The addition of studies conducted in ED settings was a protocol modification for Key Question 1 and 1a, due to few randomized controlled trials (RCTs) conducted in field settings.

- Exclude: Inpatient, clinic, or ED setting (for Key Questions other than 1 and 1a)

\section{Study Designs:}

- Randomized controlled trials

- Cohort and case-control studies

- For comparisons related to different doses, a preliminary search indicated that there are few head-to-head studies directly comparing different doses; therefore, we also included placebo-controlled studies that evaluated single doses for the purpose of potentially informing indirect comparisons related to dosing.

- For Key Question 4, we included uncontrolled longitudinal studies of patients who were successfully treated for suspected opioid overdose with naloxone in the field and not transported to a health care facility. The addition of uncontrolled studies was a protocol 
modification for Key Question 4, due to the lack of controlled studies of patients who were transported versus not transported.

\section{Data Extraction}

For each study that met inclusion criteria, a single investigator abstracted information on study design, year, setting (field or ED), country, sample size, eligibility criteria, population and clinical characteristics (age, sex, race, type of opioid involved in overdose, dose of opioid involved in overdose, presence of other drugs or substances contributing to overdose, estimated time since overdose, concomitant psychiatric comorbidities, prior overdose episodes), intervention characteristics (route of administration, dose/concentration, time to initial and repeat dosing, training/background of personnel administering drug), source of funding, and results relevant to each Key Question. We calculated relative risks and 95\% confidence intervals if necessary for included outcomes, from data reported in the studies. All data abstractions were reviewed by a second investigator for accuracy and discrepancies were resolved through discussion and consensus. See Appendix D for data abstraction of included studies.

\section{Risk of Bias Assessment of Individual Studies}

We assessed risk of bias of included studies using predefined criteria. Two investigators independently assessed risk of bias. Disagreements were resolved by consensus. Our approach for assessing risk of bias is based on the Methods Guide for Effectiveness and Comparative Effectiveness Reviews. ${ }^{52}$ We adapted criteria for assessing risk of bias from the U.S. Preventive Services Task Force. ${ }^{61}$ For RCTs, risk of bias assessment criteria included randomization and allocation concealment methods, comparability of groups at baseline, blinding, attrition, use of intention-to-treat analysis, and prespecification of outcomes. For cohort studies, assessment criteria were based on patient selection methods; comparability of groups at baseline; methods used to ascertain exposures, confounders, and outcomes; blinding of outcomes assessors; attrition and missing data; and statistical analysis of potential confounders. For uncontrolled longitudinal studies, we used the same criteria as for cohort studies, but did not assess comparability of groups at baseline or statistical adjustment for confounders. Studies were rated as "low risk of bias," "medium risk of bias," or "high risk of bias" based on the presence and seriousness of methodological shortcomings; uncontrolled studies were rated high risk of bias since they can only address the comparative effectiveness questions addressed in this review indirectly.

Studies rated "low risk of bias" are considered to have the least risk of bias, and their results are generally considered valid. "Low risk of bias" studies include clear descriptions of the population, setting, interventions, and comparison groups; a valid method for allocation of patients to treatment; low dropout rates and clear reporting of dropouts; appropriate means for preventing bias; and appropriate measurement of outcomes.

Studies rated "medium risk of bias" are susceptible to some bias, though not necessarily enough to invalidate the results. These studies may not meet all the criteria for a rating of low risk of bias, but no flaw likely to cause major bias. The study may be missing information, making it difficult to assess limitations and potential problems. The "medium risk of bias" category is broad, and studies with this rating vary in their strengths and weaknesses. The results of some medium risk of bias studies are likely to be valid, while others may be only possibly valid. 
Studies rated "high risk of bias" have significant flaws that imply biases of various types that may invalidate the results. They have a serious or "fatal" flaw in design, analysis, or reporting; large amounts of missing information; discrepancies in reporting; or serious problems in the delivery of the intervention. In general, cohort studies that do not perform adjustment for potential confounders were assessed as "high risk of bias." The results of high risk of bias studies are at least as likely to reflect flaws in the study design as the true difference between the compared interventions. We did not exclude studies rated high risk of bias a priori, but high risk of bias studies were considered to be less reliable than low or medium risk of bias studies when synthesizing the evidence, such as when discrepancies between studies are present.

See Appendix E for the risk of bias assessments of included studies.

\section{Assessing Research Applicability}

Applicability is defined as the extent to which the effects observed in published studies are likely to reflect the expected results when a specific intervention is applied to the population of interest under "real-world" conditions. ${ }^{52}$ It is an indicator of the extent to which research included in a review might be useful for informing clinical decisions in specific situations. Because applicability depends on the perspective of the user of the review, we did not assign a rating for applicability (such as "high" or "low"). We used the PICOTS framework to consider the applicability of the evidence base for each Key Question. For example, we examined the characteristics of the patient populations (e.g., age [including the proportion of patients $>65$ years of age], duration of overdose, and opioids involved in the overdose, if known [in particular, involvement of long-acting opioids or high-potency synthetic opioids]); and study setting (e.g., field versus clinical setting). Variability in the studies may limit the ability to generalize results to other populations and settings.

\section{Data Synthesis and Rating the Body of Evidence}

We constructed evidence tables with study characteristics, results, and risk of bias ratings for all included studies, and summary tables to highlight the main findings. Given the small number of studies for each Key Question and clinical and methodological heterogeneity among the studies, we determined that meta-analysis was not indicated. Rather, we synthesized studies qualitatively. We graded the strength of evidence for each Key Question and comparison for prioritized clinical outcomes (mortality, time to reversal of symptoms, recurrence of overdose symptoms, respiratory or cardiac arrest, rates/severity of drug withdrawal, and combativeness) by using the approach described in the AHRQ Methods Guide for Comparative Effectiveness and Effectiveness Reviews. ${ }^{52}$ One investigator performed the initial strength of evidence assessment and discussed with the entire team to reach consensus. Assessments were based on the following domains:

- Study limitations, based on the overall risk of bias across studies (low, medium, or high)

- Consistency of results across studies (consistent, inconsistent, or unable to determine when only one study was available)

- Directness of the evidence linking the intervention and health outcomes (direct or indirect)

- Precision of the estimate of effect, based on the number and size of studies and confidence intervals for the estimates (precise or imprecise) 
- Reporting bias, based on whether the studies defined and reported primary outcomes and whether we identified relevant unpublished studies (suspected or undetected)

Based on our assessments on the domains described above, we graded the strength of evidence for each Key Question using the four key categories recommended in the Methods Guide for Comparative Effectiveness and Effectiveness Reviews. ${ }^{52}$ Randomized controlled trials start as "high" strength of evidence and were graded down based on the presence of deficiencies in the domains. Observational studies start as low, and can be upgraded for factors such as presence of a dose-response relationship, large magnitude of effects, or plausible confounders increasing reported estimates. A "high" grade indicates high confidence that the evidence reflects the true effect and that further research is very unlikely to change our confidence in the estimate of effect. A "moderate" grade indicates moderate confidence that the evidence reflects the true effect and further research may change the estimate. A "low" grade indicates low confidence that the evidence reflects the true effect and further research is likely to change the confidence in the estimate of effect and could increase the confidence in the estimate. An "insufficient" grade indicates evidence either is unavailable or is too limited to permit any conclusion, due to extreme study limitations, inconsistency, imprecision, or reporting bias.

See Appendix F for the strength of evidence table.

\section{Peer Review and Public Commentary}

Peer reviewers and federal partners were invited to provide comments on the draft report. The AHRQ Task Order Officers and an EPC Associate Editor also provided comments and editorial review. The EPC considered comments prior to finalization of the report and completed a disposition of all peer review comments, which will be posted after publication of the final report on the AHRQ public Web site. 


\section{Results}

\section{Results of Literature Searches}

The search and selection of articles are summarized in the literature flow diagram (Figure 2). Database searches resulted in 1,934 potentially relevant articles. After dual review of abstracts and titles, 202 articles were selected for full-text dual review, and 13 studies were determined to meet inclusion criteria and were included in this review. In all studies meeting inclusion criteria, naloxone was administered by emergency medical services (EMS) personnel. No study reported funding from manufacturers of naloxone. Data extraction and risk of bias assessment tables for all included studies are available in Appendixes D and E.

\section{Figure 2. Literature flow diagram}

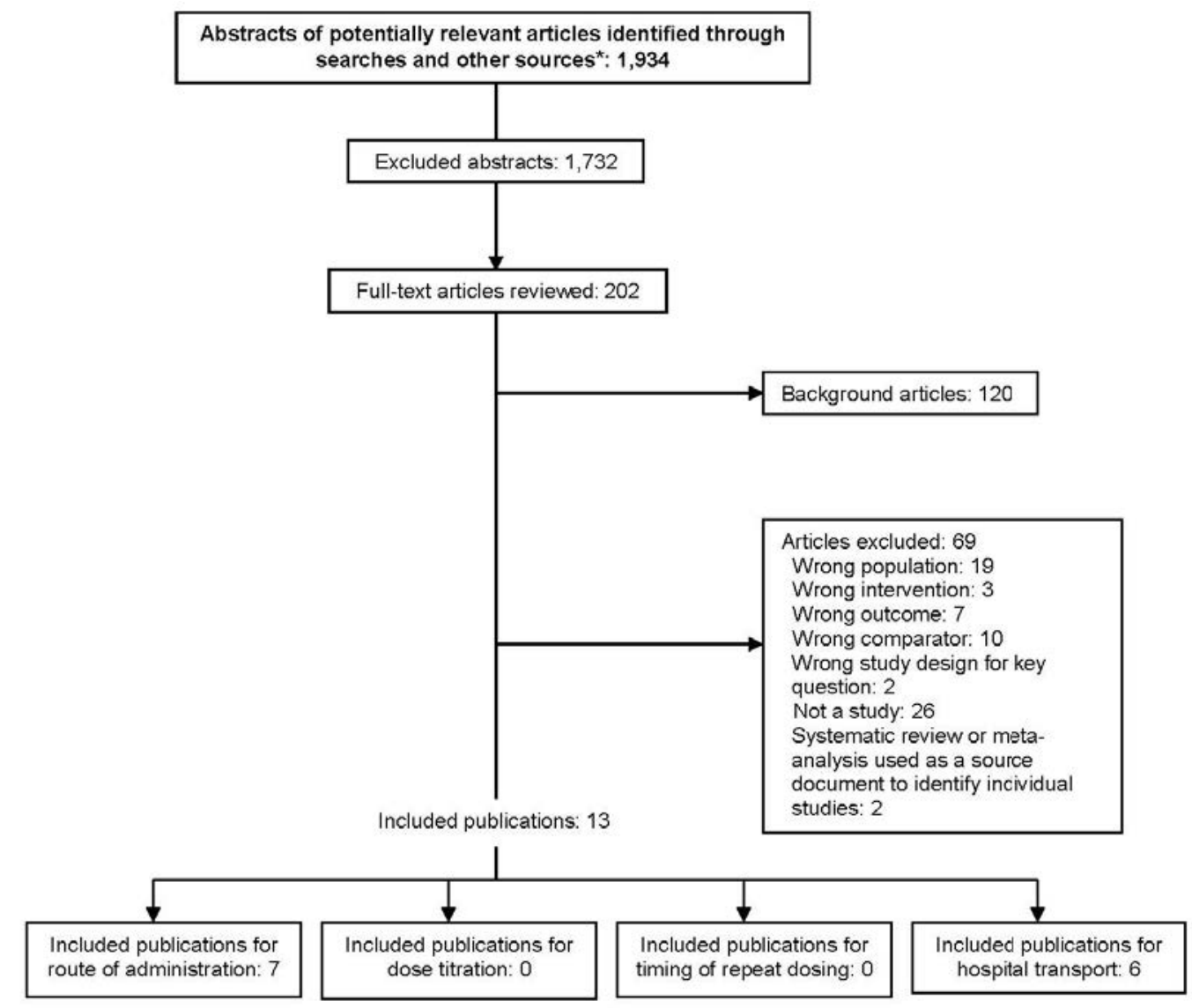

* Other sources include prior reports, references lists, referrals from experts, and grey literature 


\title{
Description of Included Studies
}

\author{
Key Question 1: For patients with confirmed or suspected opioid overdose, \\ what are the comparative benefits and harms of out-of-hospital \\ administration of naloxone by EMS personnel using intravenous, \\ intramuscular, subcutaneous, and intranasal routes of administration?
}

\section{Key Points}

- Intranasal versus intramuscular naloxone:

o One trial found no difference between intranasal (IN) naloxone (2 mg, administered as a $2 \mathrm{mg} / 1 \mathrm{~mL}$ formulation) versus intramuscular (IM) naloxone (2 $\mathrm{mg}$ ) in the likelihood of adequate response within 10 minutes (72\% vs. 78\%, adjusted Odds Ratio [OR] 0.7, 95\% confidence interval [CI] 0.3 to 1.5), mean response time (8.0 vs. 7.9 minutes), or agitation/violence (6.0\% vs. 7.9\%, Relative Risk [RR] 0.77, 95\% CI 0.25 to 2.3 ). IN naloxone was associated with increased likelihood of rescue naloxone use (18\% vs. $4.5 \%$, adjusted OR 4.8, 95\% CI 1.4 to 16).

0 Another trial found lower concentration IN naloxone (2 mg administered as a $2 \mathrm{mg} / 5$ $\mathrm{mL}$ formulation) associated with lower likelihood of spontaneous respirations within 8 minutes (63\% vs. $82 \%$, OR 0.38 , 95\% CI 0.18 to 0.81 ), higher likelihood of rescue naloxone use ( $26 \%$ vs. $13 \%$, OR 2.4 , 95\% CI 1.0 to 5.7), longer time to respirations $>10 /$ minutes ( 8 vs. 6 minutes, $\mathrm{p}=0.006$ ), and trend towards decreased likelihood of Glasgow Coma Scale (GCS) score $>11$ at 8 minutes (57\% vs. 72\%, OR 0.52, 95\% CI 0.27 to 1.0 ) than IM naloxone ( $2 \mathrm{mg}$ ). IN naloxone was associated with decreased risk of agitation and/or irritation (2.4\% vs. 14\%, RR 0.19, 95\% CI 0.04 to 0.83 ).

o The strength of evidence (SOE) for this comparison was low, due to moderate study limitations and inconsistency.

- Intranasal versus intravenous naloxone:

o One trial conducted in an Iranian emergency department (ED) setting found that IN naloxone (0.4 mg, administered as a $0.4 \mathrm{mg} / 2 \mathrm{~mL}$ formulation) was associated with a greater likelihood than intravenous (IV) naloxone $(0.4 \mathrm{mg})$ of an adequate response (defined as level of consciousness following naloxone of lethargic or conscious, $100 \%$ vs. $60 \%$, RR 1.7, 95\% CI 1.3 to 2.1 ) and lower likelihood of agitation than IV naloxone ( $0 \%$ vs. $24 \%$, RR $0.04,95 \%$ CI 0.002 to 0.66 ). The SOE was insufficient, due to moderate study limitations, inability to assess consistency, and indirectness (poor applicability to U.S. field settings due to high proportion of overdoses related to use of opium and ED setting).

o Two cohort studies reported few clear differences between IN and IV naloxone, but had serious methodological shortcomings, including failure to adjust for confounders (SOE: insufficient).

\section{Detailed Synthesis}

We identified three randomized controlled trials (RCTs) ( $n=100$ to 182) ${ }^{62-64}$ (Table 2 and Appendix D, Table D-1) and four cohort studies ( $\mathrm{n}=93$ to 609$)^{65-68}$ (Table 3 and Appendix D, Table D-2) that compared different routes of naloxone administration. Two of the RCTs, ${ }^{62,63}$ both of which were conducted in Australia, were performed in field settings and one RCT ${ }^{64}$ was 
performed in an ED setting in Iran. The field RCTs evaluated intranasal naloxone at concentrations of $2 \mathrm{mg} / 5 \mathrm{~mL}^{62}$ or $2 \mathrm{mg} / 1 \mathrm{~mL}^{63}$ administered at a dose of $1 \mathrm{mg}$ per nostril versus intramuscular naloxone $2 \mathrm{mg}$, with intramuscular naloxone $0.8 \mathrm{mg}$ administered in both groups for failure to respond after 8 minutes. None of the studies reported industry funding. The ED RCT compared intranasal naloxone $0.4 \mathrm{mg} / 2 \mathrm{~mL}$ at a dose of $0.2 \mathrm{mg}$ per nostril versus IV naloxone $0.4 \mathrm{mg}$, with repeat administration of the same dose using the same route of administration for failure to respond within 5 minutes. ${ }^{64}$ No trial evaluated the recently U.S. Food and Drug Administration (FDA)-approved formulations of IN or IM (via auto-injector) naloxone. In the field studies, concomitant drugs or alcohol were present or suspected in 30 to 50 percent of patients; the type and dose of opioid associated with the overdose episode were not reported. ${ }^{62,63}$ In the ED trial, the proportion of patients with concomitant drugs or alcohol was not reported. ${ }^{64}$ The field trials did not report the opioid involved in the overdose; in the ED trial, the opioid causing the overdose was opium in 44 to 48 percent of patients, heroin in 24 to 28 percent of patients, and methadone in 16 to 20 percent of patients; diphenoxylate, "crack" (defined in the study as heroin combined with other opioid agents), and buprenorphine were reported in small numbers of patients. All trials were rated moderate risk of bias (Appendix E). Methodological shortcomings included unblinded design and baseline between-group differences.

Two cohort studies compared IV ( 0.4 to $2.0 \mathrm{mg}$ or dose not reported) versus IN naloxone (2 mg, concentration not reported), ${ }^{65,66}$ one compared IM (2 mg) versus IV (2 mg) naloxone, ${ }^{67}$ and one compared SC $(0.8 \mathrm{mg})$ versus IV $(0.4 \mathrm{mg})$ naloxone. ${ }^{68}$ All of the studies were conducted prior to 2015, and before FDA approval of new formulations of IN and IM naloxone. Three studies were conducted in the U.S. ${ }^{65-67}$ and one study in Canada. ${ }^{68}$ No study reported the type or dose of opioid associated with the overdose, or presence of concomitant drugs or alcohol. All of the observational studies were rated high risk of bias (Appendix E). No study adjusted for potential confounders, reported missing data, or attempted to blind outcome assessors; some studies also reported important baseline between-group differences. None of the studies reported industry funding, though one study did not report a funding source.

\section{Intranasal Versus Intramuscular Naloxone}

The two field trials of IN versus IM naloxone reported somewhat inconsistent results, which could be related to the use of IN naloxone preparations with different concentrations. ${ }^{62,63}$ In the first, earlier trial, which used a less-concentrated naloxone preparation $(2 \mathrm{mg} / 5 \mathrm{~mL})$, IN naloxone was associated with lower likelihood of spontaneous respirations within 8 minutes (63\% vs. $82 \%$, OR 0.38 , 95\% CI 0.18 to 0.81 ), higher likelihood of rescue naloxone use ( $26 \%$ vs. $13 \%$, OR 2.4, 95\% CI 1.0 to 5.7), and longer time to respirations $>10$ /minutes (8 vs. 6 minutes, $\mathrm{p}=0.006$ ) than IM naloxone. ${ }^{62}$ The difference in likelihood of Glasgow Coma Scale score $>11$ at 8 minutes ( $57 \%$ vs. $72 \%$, OR $0.52,95 \%$ CI 0.27 to 1.0 ) favored IM naloxone, but was of borderline statistical significance. The $2 \mathrm{mg} / 5 \mathrm{~mL}$ formulation is lower in concentration than formulations used in the U.S. The IN naloxone was associated with decreased risk of agitation and/or irritation ( $2.4 \%$ vs. $14 \%$, RR $0.19,95 \%$ CI 0.04 to 0.83 ), with no difference in risk of nausea or vomiting, or other adverse events. In the second trial, which used a more-concentrated IN naloxone preparation ( $2 \mathrm{mg} / 1 \mathrm{~mL}$ ), there was no difference between IN versus IM naloxone in the likelihood of adequate response (defined as effective and spontaneous respirations at a rate $\geq 10$ /minute or Glasgow Coma Scale [GCS] score $\geq 13$ ) within 10 minutes ( $72 \%$ vs. $78 \%$, adjusted OR $0.7,95 \%$ CI 0.3 to 1.5 ) or mean response time ( 8.0 vs. 7.9 minutes), though IN 
naloxone was associated with increased likelihood of rescue naloxone use (18\% vs. 4.5\%, adjusted OR 4.8, 955 CI 1.4 to 16). ${ }^{63}$ There was no difference in risk of agitation and/or violence (6.0\% vs. $7.9 \%$, RR $0.77,95 \%$ CI 0.25 to 2.3 ), nausea or vomiting, or other adverse events. The $2 \mathrm{mg} / 1 \mathrm{~mL}$ formulation appears to have been manufactured specifically for this trial, and is not available in any product otherwise.

\section{Intranasal Versus Intravenous Naloxone}

One $\mathrm{RCT}^{64}$ and two observational studies ${ }^{65,66}$ evaluated IN versus IV naloxone. The RCT, conducted in an ED setting, found IN naloxone associated with greater likelihood than IV naloxone of an adequate response (defined as level of consciousness following naloxone of lethargic or conscious, $100 \%$ vs. $60 \%$, RR 1.7, 95\% CI 1.3 to 2.1). ${ }^{64}$ IN naloxone was also associated with greater likelihood of achieving full consciousness (44\% vs. 24\%, RR 1.8, 95\% CI 1.0 to 3.3). The mean response time was longer with IN than IV naloxone by about 1 minute (2.56 vs. 1.48 minutes, $\mathrm{p}<0.001$ ), with no difference in length of hospital stay (1.53 vs. 1.2 days, $\mathrm{p}=0.15)$. However, the point from which response time was measured was not clear. The IN naloxone was associated with lower likelihood of agitation than IV naloxone ( $0 \%$ vs. 24\%, RR $0.04,95 \%$ CI 0.002 to 0.66$)$.

The two cohort studies of IN versus IV naloxone reported few clear differences. ${ }^{65,66}$ One study found no difference in median change in breaths per minute ( 4 vs. $6, \mathrm{p}=0.08$ ) or Glasgow Coma Scale score ( 3 vs. $4, \mathrm{p}=0.19$ ) from baseline. ${ }^{65}$ The other found no difference in likelihood of a positive clinical response (increase in respirations of $\geq 6$ breaths/minute or Glasgow Coma Scale score $\geq 6$ points) ( $66 \%$ vs. $56 \%, p=0.3$ ) or time from patient contact to effect ( $20 \mathrm{vs.} 21$ minutes, $\mathrm{p}=0.9)$, though IN naloxone was associated with longer time from administration to effect (13 vs. 8.1 minutes, $\mathrm{p}=0.3){ }^{66}$

\section{Other Route of Administration Comparisons}

Other comparisons involving different routes of naloxone administration were evaluated in one cohort study each. One cohort study found no difference between IM versus IV naloxone in likelihood of a positive response (GCS $\geq 14$ and respiratory rate $\geq 10$ /minute within 5 minutes of naloxone administration) (94\% vs. 95\%, RR 1.0, 95\% CI 0.94 to 1.1 ). ${ }^{67}$ Another cohort study found SC naloxone associated with longer time from administration to respiratory rate $\geq 10$ /minute ( 5.5 vs. 3.8 minutes, $p=0.001$ ) than IV naloxone, but no difference in time from arrival at patient's side to respiratory rate $\geq 10$ /minute (9.6 vs. 9.3 minutes, $p=0.67$ ). ${ }^{68}$ The SC naloxone was associated with lower likelihood than IV naloxone of requiring multiple doses (15\% vs. 35\%, RR 0.42, 95\% CI 0.25 to 0.71 ).

\section{Subgroup Effects}

No study evaluated how comparative benefits and harms of different routes of naloxone administration differed according to age, sex, race, type of opioid involved in overdose, dose of opioid involved in overdose, presence of other drugs or substances contributing to overdose, estimated time since overdose, concomitant psychiatric comorbidities, or prior overdose episodes. Two trials ${ }^{62,63}$ and one cohort study ${ }^{65}$ reported that naloxone was administered by paramedics or Advanced Life Support personnel; one cohort study ${ }^{68}$ reported the level of training of EMS personnel as ranging from Basic to Advanced Life Support, but it was not clear who administered the naloxone. There was insufficient evidence to determine how level of EMS training affected findings regarding route of administration comparisons. 
Table 2. Characteristics and results of randomized controlled trials comparing routes of naloxone administration

\begin{tabular}{|c|c|c|c|c|}
\hline $\begin{array}{l}\text { Author, Year } \\
\text { Country } \\
\text { Risk of Bias }\end{array}$ & $\begin{array}{l}\text { Intervention } \\
\text { (Sample Size) }\end{array}$ & $\begin{array}{l}\text { Population } \\
\text { Characteristics by } \\
\text { Treatment Group }\end{array}$ & Results & Adverse Events \\
\hline $\begin{array}{l}\text { Kelly, } 2005^{62} \\
\text { Australia } \\
\text { Medium }\end{array}$ & $\begin{array}{l}\text { A: Naloxone } \\
\text { intranasal } 2 \mathrm{mg} / 5 \mathrm{~mL} \\
\text { with mucosal } \\
\text { atomization device, } 1 \\
\text { mg each nostril } \\
(\mathrm{n}=84) \\
\text { B: Naloxone } \\
\text { intramuscular } 2 \mathrm{mg} x \\
1 \text { (preparation not } \\
\text { described) ( } \mathrm{n}=71) \\
\text { Paramedics } \\
\text { administered } \\
\text { naloxone. } \\
\text { Both groups } \\
\text { received naloxone } \\
\text { intramuscular } 0.8 \mathrm{mg} \\
\text { for failure to respond } \\
\text { after } 8 \text { minutes }\end{array}$ & $\begin{array}{l}\text { Age: } 28 \text { vs. } 30 \\
\text { years } \\
\text { Male: } 70 \% \text { vs. } 73 \% \\
\text { Race: Not reported } \\
\text { Psychiatric } \\
\text { comorbidities: Not } \\
\text { reported } \\
\text { Prior overdose } \\
\text { episodes: Not } \\
\text { reported }\end{array}$ & 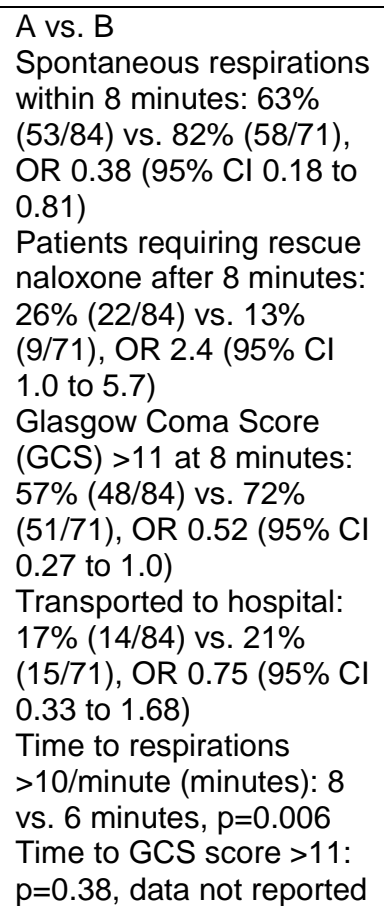 & 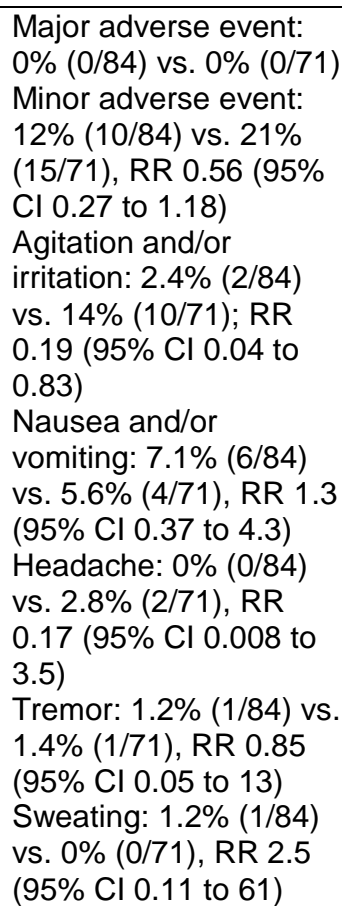 \\
\hline $\begin{array}{l}\text { Kerr, } 2009^{63} \\
\text { Australia } \\
\text { Medium }\end{array}$ & $\begin{array}{l}\text { A: Naloxone } \\
\text { intranasal } 2 \mathrm{mg} / 1 \mathrm{~mL} \\
\text { with mucosal } \\
\text { atomization device, } 1 \\
\mathrm{mg} \text { each nostril } \\
(\mathrm{n}=83 \text { ) } \\
\text { B: Naloxone } \\
\text { intramuscular } 2 \mathrm{mg} / 5 \\
\mathrm{~mL} \text { 'min-i-jet' } \\
\text { preparation x } 1 \\
\text { (n=89) } \\
\text { Paramedics } \\
\text { administered } \\
\text { naloxone. } \\
\text { Both groups } \\
\text { received naloxone } \\
\text { intramuscular } 0.8 \mathrm{mg} \\
\text { for failure to respond } \\
\text { after } 10 \text { minutes }\end{array}$ & $\begin{array}{l}\text { Age: } 31 \text { vs. } 32 \\
\text { years } \\
\text { Male: } 77 \% \text { vs. } 71 \% \\
\text { Race: Not reported } \\
\text { Psychiatric } \\
\text { comorbidities: Not } \\
\text { reported } \\
\text { Prior overdose } \\
\text { episodes: Not } \\
\text { reported }\end{array}$ & 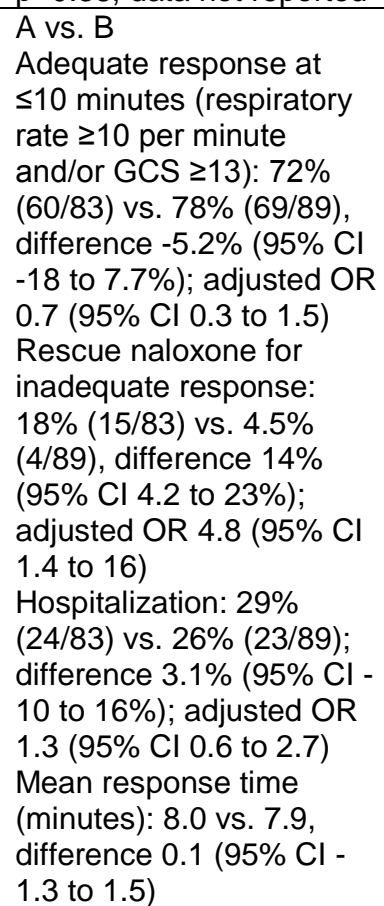 & 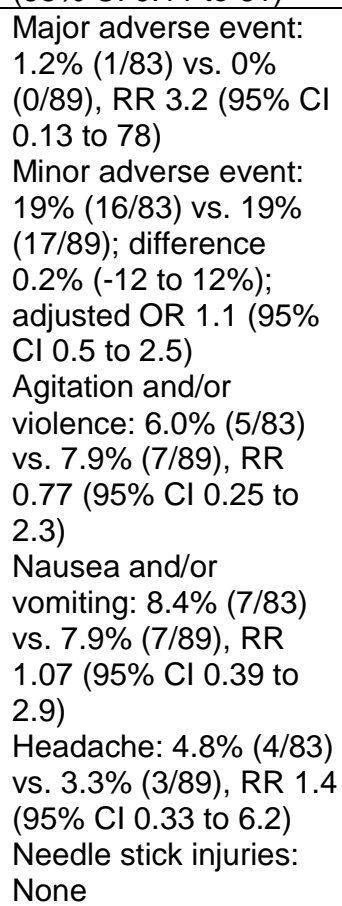 \\
\hline
\end{tabular}




\begin{tabular}{|c|c|c|c|c|}
\hline $\begin{array}{l}\text { Author, Year } \\
\text { Country } \\
\text { Risk of Bias }\end{array}$ & $\begin{array}{l}\text { Intervention } \\
\text { (Sample Size) }\end{array}$ & $\begin{array}{l}\text { Population } \\
\text { Characteristics by } \\
\text { Treatment Group }\end{array}$ & Results & Adverse Events \\
\hline $\begin{array}{l}\text { Sabzghabaee, } \\
2014^{64} \\
\text { Iran } \\
\text { High }\end{array}$ & $\begin{array}{l}\text { A: Naloxone } \\
\text { intranasal } 0.4 \mathrm{mg} \text { in } \\
2 \mathrm{~mL}(\mathrm{n}=50) \\
\mathrm{B} \text { : Naloxone } \\
\text { intravenous } 0.4 \mathrm{mg} \\
(\mathrm{n}=50) \\
\text { All patients who } \\
\text { failed to respond } \\
\text { within } 5 \text { minutes } \\
\text { were given a further } \\
0.4 \text { mg naloxone by } \\
\text { the same } \\
\text { administration route } \\
\text { as the first dose }\end{array}$ & $\begin{array}{l}\text { Age: } 30 \text { vs. } 33 \\
\text { years } \\
\text { Male: } 78 \% \text { vs. } 74 \% \\
\text { Race: Not reported } \\
\text { Psychiatric } \\
\text { comorbidities: Not } \\
\text { reported } \\
\text { Prior overdose } \\
\text { episodes: Not } \\
\text { reported }\end{array}$ & $\begin{array}{l}\text { A vs. B: } \\
\text { Systolic blood pressure: } \\
106 \pm 14.7 \text { vs. } 112 \pm 9.6, \\
p=0.18 \\
\text { Diastolic blood pressure: } \\
78 \pm 7.1 \text { vs. } 77 \pm 4.5, p=0.18 \\
\text { Heart rate (per minute): } \\
90 \pm 8.3 \text { vs. } 97 \pm 12.9, \\
p=0.003 \\
\text { Respirator rate (per } \\
\text { minute): } 18 \pm 2.4 \text { vs. } \\
19 \pm 2.8, p=0.17 \\
\text { Glasgow Coma Scale: } \\
14.3 \pm 0.73 \text { vs. } 13.2 \pm 1.5 \\
p<0.001 \\
\text { Length of hospital stay } \\
\text { (days): } 1.53 \pm 0.16 \text { vs. } \\
1.2 \pm 0.15, p=0.15\end{array}$ & Agitation: 0 vs. 12 \\
\hline
\end{tabular}

CI = confidence interval; GCS = Glasgow Coma Scale; OR = odds ratio; RR = relative risk

Table 3. Characteristics and results of cohort studies comparing routes of naloxone administration

\begin{tabular}{|c|c|c|c|}
\hline $\begin{array}{l}\text { Author, } \\
\text { Year } \\
\text { Country } \\
\text { Risk of Bias }\end{array}$ & $\begin{array}{l}\text { Comparison Group } \\
\text { (Sample Size) }\end{array}$ & $\begin{array}{l}\text { Population Characteristics by } \\
\text { Comparison Group }\end{array}$ & Results \\
\hline $\begin{array}{l}\text { Merlin, } \\
2010^{65} \\
\text { United } \\
\text { States } \\
\text { High }\end{array}$ & $\begin{array}{l}\text { A: Intravenous naloxone } \\
\text { between } 0.4 \text { and } 2.0 \mathrm{mg} \\
(\mathrm{n}=55) \\
\text { B: Intranasal naloxone at } 1 \\
\text { mg per nostril at the } \\
\text { discretion of the paramedics } \\
(\mathrm{n}=38) \\
\text { Naloxone administered by } \\
\text { Advanced Life Support } \\
\text { personnel }\end{array}$ & $\begin{array}{l}\text { Age: } 42 \text { vs. } 38 \text { years } \\
\text { Male: } 67 \% \text { vs. } 61 \% \\
\text { Race: Not reported } \\
\text { Psychiatric comorbidities: Not } \\
\text { reported } \\
\text { Prior overdose episodes: Not } \\
\text { reported } \\
\text { Receipt of out-of-hospital } \\
\text { naloxone: } 100 \% \text { vs. } 100 \%\end{array}$ & $\begin{array}{l}\text { A vs. B: } \\
\text { Median change in breaths per } \\
\text { minute from baseline: } 6 \text { vs. } 4 \text {, } \\
\text { Difference } 2 \text { ( } 95 \% \mathrm{Cl}-0.001 \text { to } \\
5), p=0.08 \\
\text { Median change in Glasgow } \\
\text { Coma Scale (GCS) from } \\
\text { baseline: } 4 \text { vs. 3, Difference } 1 \\
(95 \% \mathrm{Cl}-0.001 \text { to 3), } p=0.19\end{array}$ \\
\hline $\begin{array}{l}\text { Robertson, } \\
2009^{66} \\
\text { United } \\
\text { States } \\
\text { High }\end{array}$ & $\begin{array}{l}\text { A: Intravenous naloxone as } \\
\text { first line treatment, dose } \\
\text { protocol not reported ( } n=104) \\
\text { B: Intranasal naloxone as first } \\
\text { line treatment, } 2 \mathrm{mg} \mathrm{(1} \mathrm{mg} \\
\text { per nostril) using mucosal } \\
\text { atomizer device ( } \mathrm{n}=50) \\
\text { EMS administered naloxone, } \\
\text { training not specified } \\
\text { Patients in first year were } \\
\text { treated with intravenous } \\
\text { naloxone and patients in last } \\
5 \text { months were treated with } \\
\text { intranasal naloxone. }\end{array}$ & $\begin{array}{l}\text { Age: } 44 \text { vs. } 41 \text { years } \\
\text { Male: } 60 \% \text { vs. } 71 \% \\
\text { Race: Not reported } \\
\text { Psychiatric comorbidities: Not } \\
\text { reported } \\
\text { Prior overdose episodes: Not } \\
\text { reported } \\
\text { Receipt of out-of-hospital } \\
\text { naloxone: } 100 \% \text { vs. } 100 \%\end{array}$ & $\begin{array}{l}\text { A vs. B: } \\
\text { Positive clinical response } \\
\text { (increase in respirations of at } \\
\text { least } 6 \text { breaths/minute or in GCS } \\
\text { of at least } 6 \text { points): } 58 / 104 \\
\text { ( } 56 \% \text { ) vs. } 33 / 50(66 \%), p=0.3 \\
\text { Time from naloxone } \\
\text { administration to effect: } 8.1 \text { vs. } \\
12.9 \text { minutes, } p=0.02 \\
\text { Time from patient contact to } \\
\text { effect: } 20.7 \text { vs. } 20.3 \text { minutes, } \\
p=0.9 \\
\text { Second dose of naloxone given: } \\
19 / 104(18 \%) \text { vs. } 17 / 50 \text { (34\%), } \\
p=0.05\end{array}$ \\
\hline
\end{tabular}




\begin{tabular}{|c|c|c|c|}
\hline $\begin{array}{l}\text { Author, } \\
\text { Year } \\
\text { Country } \\
\text { Risk of Bias }\end{array}$ & $\begin{array}{l}\text { Comparison Group } \\
\text { (Sample Size) }\end{array}$ & $\begin{array}{l}\text { Population Characteristics by } \\
\text { Comparison Group }\end{array}$ & Results \\
\hline $\begin{array}{l}\text { Sporer, } \\
1996^{67} \\
\text { United } \\
\text { States } \\
\text { High }\end{array}$ & $\begin{array}{l}\text { A: Intravenous naloxone } 2 \\
\mathrm{mg}(\mathrm{n}=69) \\
\mathrm{B} \text { : Intramuscular naloxone } 2 \\
\mathrm{mg}(\mathrm{n}=487) \\
\text { Repeat dose given if no } \\
\text { response is seen in } 1-2 \\
\text { minutes }\end{array}$ & $\begin{array}{l}\text { Not reported by treatment group } \\
\text { Age: <20: 3\%; 20-29: 15\%; 30- } \\
\text { 39: 40\%; 40-49: 34\%; >50: 9\% } \\
\text { Male: 78\% } \\
\text { Race: Unknown: 51\%; White: } \\
\text { 28\%; Black: 14\%; Hispanic: 6\%; } \\
\text { Asian: 1\% } \\
\text { Psychiatric comorbidities: Not } \\
\text { reported } \\
\text { Prior overdose episodes: Not } \\
\text { reported } \\
\text { Receipt of out-of-hospital } \\
\text { naloxone: } 99.8 \%\end{array}$ & $\begin{array}{l}\text { A vs. B: } \\
\text { Response rate (GCS } \geq 14 \text { and } \\
\text { RR } \geq 10 / \text { minute within } 5 \text { minutes } \\
\text { of naloxone administration): } \\
488 / 518(94 \%) \text { vs. } 69 / 73(95 \%) \text {, } \\
\text { RR } 1.0 \text { ( } 95 \% \mathrm{Cl} 0.94 \text { to } 1.1)\end{array}$ \\
\hline $\begin{array}{l}\text { Wanger, } \\
1998^{68} \\
\text { Canada } \\
\text { High }\end{array}$ & $\begin{array}{l}\text { A: Subcutaneous naloxone } \\
0.8 \text { mg into upper arm or } \\
\text { thigh, with } 0.4 \text { mg IV } \\
\text { available if no improvement } \\
\text { after } 5 \text { minutes ( } \mathrm{n}=139 \text { ) } \\
\text { B: Intravenous naloxone } 0.4 \\
\text { mg, with an additional } 0.4 \mathrm{mg} \\
\text { available if no improvement } \\
\text { after } 5 \text { minutes ( } \mathrm{n}=83 \text { ) } \\
\text { Overdose treated by British } \\
\text { Columbia Ambulance Service } \\
\text { attendants (basic life support, } \\
\text { IV and advanced life support } \\
\text { levels); unclear who } \\
\text { administered naloxone }\end{array}$ & $\begin{array}{l}\text { A vs. B } \\
\text { Age: } 33 \text { vs. } 36 \text { years } \\
\text { Male: } 80 \% \text { vs. } 78 \% \\
\text { Race: Not reported } \\
\text { Psychiatric comorbidities: Not } \\
\text { reported } \\
\text { Prior overdose episodes: Not } \\
\text { reported } \\
\text { Receipt of out-of-hospital } \\
\text { naloxone: } 100 \%\end{array}$ & $\begin{array}{l}\text { Time interval from drug } \\
\text { administration to respiratory rate } \\
\geq 10 \text { breaths per minute: } 5.5 \text { vs. } \\
3.8 \text { minutes, } p=0.001 \\
\text { Time interval from arrival at } \\
\text { patient's side to respiratory rate } \\
\geq 10 \text { breaths per minute: } 9.6 \text { vs. } \\
9.3 \text { minutes, } p=0.67 \\
\text { Proportion requiring multiple } \\
\text { naloxone doses: } 15 \%(18 / 122) \\
\text { vs. } 35 \%(26 / 74), \text { RR } 0.42(95 \% \\
\text { Cl } 0.25 \text { to } 0.71)\end{array}$ \\
\hline
\end{tabular}

CI = confidence interval; GCS = Glasgow Coma Scale; IV = intravenous; RR = relative risk

Key Question 1a: For patients with confirmed or suspected opioid overdose who receive naloxone in the out-of-hospital setting from EMS personnel, what are the comparative benefits and harms of different intravenous, intramuscular, subcutaneous, or intranasal doses of naloxone?

\section{Key Point}

- No study compared different doses of naloxone administered via the same route; there was too much clinical heterogeneity to determine effects of dose from indirect comparisons (SOE: insufficient).

\section{Detailed Synthesis}

No study compared different doses of naloxone administered via the same route. In the trials of different naloxone routes of administration described in Key Question 1, the doses, concentration of formulation, and routes of administration varied, making it difficult to interpret potential differences based on indirect comparisons.

In two RCTs of IN versus IM naloxone, the concentration of IN naloxone was $2 \mathrm{mg} / 5 \mathrm{~mL}$ in one trial and $2 \mathrm{mg} / 1 \mathrm{~mL}$ in the other, though the total dose administered $(2 \mathrm{mg})$ was the same ${ }^{62,63}$ (Table 1). The difference in concentration could help explain the inconsistency in trial results- 
the less-concentrated formulation of IN naloxone was associated with worse outcomes than IM naloxone, probably related to the ability of each nostril to absorb a maximum volumes of $\leq 0.5$ $\mathrm{mL}$. Therefore, the effective dose of naloxone was probably lower in this trial than in the trial that evaluated the higher concentration formulation, even though the total administered dose was the same. No study evaluated the new FDA-approved, highly-concentrated reformulation of naloxone (4 mg/0.1 mL).

In one RCT ${ }^{64}$ and two cohort studies ${ }^{65,66}$ of IN versus IV naloxone, the doses and formulations of naloxone varied or were not well described (Tables 1 and 2). The RCT compared intranasal naloxone $0.4 \mathrm{mg} / 2 \mathrm{~mL}$ at a dose of $0.2 \mathrm{mg}$ per nostril versus IV naloxone $0.4 \mathrm{mg}$; the two observational studies did not report the concentration of intranasal naloxone formulation (dose $2 \mathrm{mg}$ ) and the dose of IV naloxone ranged from 0.4 to $2.0 \mathrm{mg}$ or was not reported.

Therefore, no conclusions could be drawn regarding the association between naloxone dose for each route of administration and outcomes.

Key Question 2: For patients with confirmed or suspected opioid overdose in out-of-hospital settings, what are the comparative benefits and harms of titration of naloxone administered by EMS personnel until the patient resumes sufficient spontaneous respiratory effort versus until the patient regains consciousness?

\section{Key Point}

- No study compared benefits and harms of titration of naloxone until the patient resumes sufficient spontaneous respiratory effort versus until the patient regains consciousness (SOE: insufficient).

\section{Detailed Synthesis}

No study compared benefits and harms of titration of naloxone until the patient resumes sufficient spontaneous respiratory effort versus until the patient regains consciousness. One RCT defined a response to naloxone not requiring repeat administration as improvement in respiratory rate $\geq 10$ and Glasgow Coma Scale score $\geq 13 .{ }^{63}$ Other studies that compared different routes of naloxone administration did not specify titration targets, or targets were unclear. Therefore, there was insufficient indirect evidence to assess effects of different titration strategies on effectiveness.

Key Question 3: For patients with confirmed or suspected opioid overdose in out-of-hospital settings treated with multiple doses of naloxone (including patients who do not improve after an initial dose of intranasal naloxone), what are the effects on benefits and harms of differences in timing of repeat dosing?

\section{Key Point}

- No study compared benefits and harms of differences in timing of repeat dosing of naloxone (SOE: insufficient). 


\section{Detailed Synthesis}

No study compared benefits and harms of differences in timing of repeat dosing of naloxone. Among five studies that compared different routes of naloxone administration, timing of repeat dosing was 1 to 2 minutes in one study ${ }^{67}$ and 5 to 10 minutes in the others; due to other differences between trials (e.g. different doses and formulations, routes of administration, study design), it was not possible to assess effects of differences in redosing interval on effectiveness.

\section{Key Question 4: For patients with confirmed or suspected opioid overdose in out-of-hospital settings who regain sufficient spontaneous respiratory effort and are alert and oriented after naloxone administration by EMS personnel, what are the benefits and harms of transporting patients to a health care facility versus nontransport?}

\section{Key Points}

- Among patients who were successfully treated for opioid overdose by naloxone in the field and not transported to a hospital, uncontrolled studies reported rates of deaths within 0 to 2 days were 0 percent in three studies (total $N=1867), 0.6$ percent $(14 / 2241)$ in one study and 0.49 percent $(1 / 205)$ in another study; one study reported one case of a lifethreatening adverse event (1.25\% [1/84]) (SOE: insufficient).

- No study evaluated outcomes such as linkage to treatment for opioid use disorder or subsequent repeat opioid overdose episodes.

\section{Detailed Synthesis}

No study compared outcomes among patients with confirmed or suspected opioid overdose who responded to naloxone administration and were transported to a health care facility versus not transported. Six studies ( $n=84$ to 2241) reported on outcomes in patients who received naloxone for opioid overdose who were not transported to a health care facility (Table 4 and Appendix D, Table D-3). One study was conducted in Finland, ${ }^{69}$ one in The Netherlands ${ }^{70}$ and the others in the United States. None of the studies reported industry funding, though four of five studies did not report funding source. In the U.S. studies, patients declined transport to a hospital. In the European studies, reasons for nontransport were unclear or could vary (physician discretion or patient refusal). Ascertainment of naloxone administration was based on a retrospective review of EMS records and medical examiner databases, cardiac arrest registries and/or hospital records were used to assess outcomes. The studies did not describe how long patients were observed following successful reversal or opioid overdose. All of the studies were rated high risk of bias due to uncontrolled design, failure to blind outcome assessors to exposure status, and failure to report missing data (Appendix E). The uncontrolled design makes interpretation of findings difficult, since patients who refuse transport are likely to differ from those who are transported in important ways. For example, one study reported that 100 percent of patients who were not transported had Glasgow Coma Scale scores of 14 or 15, compared with about 50 percent of patients who were transported to an emergency department. ${ }^{69}$ Another methodological shortcoming is that the studies relied on medical examiner or hospital records to identify serious harms, rather than seeking to identify patients for followup. Reliance on medical examiner or hospital records requires that patients be accurately identified at the time of 
naloxone administration as well as at the time of death and that they remain in the same geographic area.

Rates of serious adverse events, including deaths, were low in all of the studies (Table 3). Within 0 to 2 days of naloxone administration, the proportion of patients was 0 percent in three studies $^{71-73}\left(n=317,552\right.$, and 998), one study ${ }^{74}$ reporting one death $(0.49 \%$ [1/205]), and one study $^{70}$ reporting 14 deaths $(0.6 \%$ [14/2241]): three attributed to likely rebound overdose $(0.13 \%)$, ten to new overdose, and one to a natural death. The final study reported one case of a life-threatening adverse event $\left(1.2 \%\right.$ [1/84]). ${ }^{69}$ One study ${ }^{74}$ reported two additional deaths at 30 days (1\% [2/205]) and another ${ }^{73}$ found nine deaths through up to 1 year (1.6\% [9/552]). No study evaluated outcomes such as linkage to treatment for opioid use disorder or subsequent repeat opioid overdose episodes.

Table 4. Characteristics and results of uncontrolled studies of patients not transported to hospitals after naloxone administration

\begin{tabular}{|c|c|c|c|}
\hline $\begin{array}{l}\text { Author, } \\
\text { Year } \\
\text { Country } \\
\text { Risk of Bias }\end{array}$ & $\begin{array}{l}\text { Comparison Group } \\
\text { (Sample Size) }\end{array}$ & $\begin{array}{l}\text { Population Characteristics by } \\
\text { Comparison Group }\end{array}$ & Results \\
\hline $\begin{array}{l}\text { Boyd, } 2006^{69} \\
\text { Finland } \\
\text { High }\end{array}$ & $\begin{array}{l}\text { Refused transport to } \\
\text { emergency department } \\
(n=84)\end{array}$ & $\begin{array}{l}\text { Overall population } \\
\text { characteristics (includes patients } \\
\text { who were transported to the } \\
\text { emergency department) } \\
\text { Age: } 26 \text { years } \\
\text { Male: } 83 \% \\
\text { Race: Not reported } \\
\text { Psychiatric comorbidities: Not } \\
\text { reported } \\
\text { Prior overdose episodes: Not } \\
\text { reported } \\
\text { Received out-of-hospital } \\
\text { naloxone: } 85 \% \text { vs. } 85 \% \\
\end{array}$ & $\begin{array}{l}\text { Life-threatening adverse event } \\
\text { within } 12 \text { hours: } 1 / 84(1.19 \% \text {, } \\
95 \% \mathrm{Cl} 0.21 \text { to } 6.44)\end{array}$ \\
\hline $\begin{array}{l}\text { Levine, } \\
2016^{74} \\
\text { United } \\
\text { States } \\
\text { High }\end{array}$ & $\begin{array}{l}\text { Refused Transport to } \\
\text { emergency department } \\
(n=205)\end{array}$ & $\begin{array}{l}\text { Age: } 41 \text { years (29-53) } \\
\text { Male: } 87 \% \\
\text { Race: Not reported } \\
\text { Psychiatric Comorbidities: Not } \\
\text { reported } \\
\text { Prior Overdoses: Not reported }\end{array}$ & $\begin{array}{l}\text { Death within } 24 \text { hours:1/205 } \\
(0.49 \%, 95 \% \mathrm{Cl} 0.09-2.71 \%) \\
\text { Additional deaths within } 30 \\
\text { days: } 2 / 205(0.98 \%, 95 \% \mathrm{Cl} \\
0.27-3.49 \%) \\
\text { Additional deaths within } 6 \\
\text { months: None }\end{array}$ \\
\hline $\begin{array}{l}\text { Rudolph, } \\
2011^{70} \\
\text { The } \\
\text { Netherlands } \\
\text { High }\end{array}$ & $\begin{array}{l}\text { Physician released patient at } \\
\text { scene or patient refused } \\
\text { transport to emergency } \\
\text { department } \\
(n=2241)\end{array}$ & $\begin{array}{l}\text { Age: Not reported } \\
\text { Sex: Not reported } \\
\text { Race: Not reported } \\
\text { Psychiatric Comorbidities: Not } \\
\text { reported } \\
\text { Prior Overdoses: Not reported }\end{array}$ & $\begin{array}{l}\text { Death within } 48 \text { hours: } 14 / 2241 \\
(0.62 \%, 95 \% \mathrm{CI} 0.37-1.04 \%) ; 3 \\
\text { likely rebound overdoses, } 10 \\
\text { new overdose, } 1 \text { natural death }\end{array}$ \\
\hline $\begin{array}{l}\text { Vilke, } 1999^{71} \\
\text { United } \\
\text { States } \\
\text { High }\end{array}$ & $\begin{array}{l}\text { Refused transport to } \\
\text { emergency department } \\
(n=317)\end{array}$ & $\begin{array}{l}\text { Age: } 37 \text { Years (18-79) } \\
\text { Male: } 82.7 \% \\
\text { Race: Not reported } \\
\text { Psychiatric Comorbidities: Not } \\
\text { reported } \\
\text { Prior Overdoses: Not reported }\end{array}$ & $\begin{array}{l}\text { Death within } 12 \text { hours: } 0 \text { (95\% } \\
\mathrm{Cl} 0 \text { to } 1.2)\end{array}$ \\
\hline $\begin{array}{l}\text { Vilke, } 2003^{72} \\
\text { United } \\
\text { States } \\
\text { High }\end{array}$ & $\begin{array}{l}\text { Refused transport to } \\
\text { emergency department } \\
(n=998)\end{array}$ & $\begin{array}{l}\text { Age: } 37.7 \text { years (16-83) } \\
\text { Male: } 83.8 \% \\
\text { Race: Not reported } \\
\text { Psychiatric Comorbidities: Not } \\
\text { reported } \\
\text { Prior Overdoses: Not reported }\end{array}$ & $\begin{array}{l}\text { Death within } 12 \text { hours: 0/998 } \\
(0 \%, 95 \% \mathrm{Cl} 0-0.38 \%)\end{array}$ \\
\hline
\end{tabular}




\begin{tabular}{|c|c|c|c|}
\hline $\begin{array}{l}\text { Author, } \\
\text { Year } \\
\text { Country } \\
\text { Risk of Bias }\end{array}$ & $\begin{array}{l}\text { Comparison Group } \\
\text { (Sample Size) }\end{array}$ & $\begin{array}{l}\text { Population Characteristics by } \\
\text { Comparison Group }\end{array}$ & Results \\
\hline $\begin{array}{l}\text { Wampler, } \\
2011^{73} \\
\text { United } \\
\text { States } \\
\text { High }\end{array}$ & $\begin{array}{l}\text { Refused transport to } \\
\text { emergency department }(n= \\
552)\end{array}$ & $\begin{array}{l}\text { Age: } 38 \pm 15 \text { years }(13-91) \\
\text { Male: } 72 \% \\
\text { Race: Not reported } \\
\text { Psychiatric Comorbidities: Not } \\
\text { reported } \\
\text { Prior Overdoses: Not reported }\end{array}$ & $\begin{array}{l}\text { Deaths within } 48 \text { hours: } 0 / 552 \\
\text { (0\%, } 95 \% \mathrm{Cl} 0-0.69 \%) \\
\text { Deaths from } 4-372 \text { days: } 9 / 592 \\
(1.6 \%, 95 \% \mathrm{Cl} 0.5 \% \text { to } 2.7 \%)\end{array}$ \\
\hline
\end{tabular}

$\mathrm{CI}$ = confidence interval 


\section{Discussion}

\section{Key Findings and Strength of Evidence}

While naloxone is generally effective for reversing suspected opioid overdose, evidence to inform optimal management of suspected opioid overdose with naloxone by emergency medical services (EMS) personnel is limited (Appendix F). Three randomized controlled trials (RCTs) directly compared different routes of naloxone administration, but all had methodological

shortcomings, including use of unblinded design. ${ }^{62-64}$ Among the three trials, two compared intranasal (IN) with intramuscular (IM) naloxone ${ }^{62,63}$ and one trial evaluated IN versus intravenous (IV) naloxone. ${ }^{64}$ No trial evaluated the recently U.S. Food and Drug Administration (FDA)-approved naloxone auto-injectors for IM administration or highly-concentrated (4 mg/0.1 $\mathrm{mL}$ or $2 \mathrm{mg} / 0.1 \mathrm{~mL}$ ) IN formulations of naloxone.

For IN versus IM naloxone, results suggest that a higher concentration formulation of IN (2 $\mathrm{mg} / 1 \mathrm{~mL}$ ) naloxone is similar in efficacy to IM naloxone ${ }^{62,63}$ (strength of evidence [SOE]: low). Although $2 \mathrm{mg}$ of IN naloxone was administered in both trials, one trial evaluated a lower concentration formulation (total of $5 \mathrm{~mL}$ instilled), ${ }^{62}$ compared with a higher concentration formulation (total of $1 \mathrm{~mL}$ instilled) in the other trial. ${ }^{63}$ In the trial that used a more-concentrated formulation of IN naloxone, there was no difference versus IM naloxone in the likelihood of an adequate response to naloxone (reversal of overdose symptoms with resumption of spontaneous respirations or level of consciousness), mean response time, or adverse events, though IN naloxone was associated with increased likelihood of rescue naloxone use (18\% vs. 4.5\%, adjusted OR 4.8, 95\% CI 1.4 to 16) ${ }^{63}$ (SOE: low). By contrast, in the trial that used a lessconcentrated formulation, IN naloxone was associated with lower response rates, longer time to response, and decreased risk of agitation and/or irritation compared with IM naloxone. ${ }^{62}$ This could be due to a lower effective dose of IN naloxone in the latter trial, since the ideal volume of delivery via the IN route is $\leq 0.5 \mathrm{~mL}$ per nostril. Efficacy was based on effects on overdose symptoms (likelihood of an adequate response, time to reversal, and recurrence of symptoms). The trials did not evaluate effects on healthcare utilization or other clinical outcomes (e.g., function or quality of life) and were not designed to assess risk of serious injuries, such as needle stick, though no cases were reported.

While naloxone is generally effective for reversing suspected opioid overdose, evidence regarding other route of administration comparisons is even more limited. One trial of IN versus IV naloxone found IN naloxone associated with greater likelihood than IV naloxone of an adequate response (defined as level of consciousness "lethargic” or “conscious," 100\% vs. 60\%, RR 1.3 to 2.1), slightly longer response time (difference $\sim 1$ minute), and lower likelihood of agitation (0\% vs. 24\%, RR 0.04, 95\% CI 0.002 to 0.66$).{ }^{64}$ However, the trial was conducted in an Iranian emergency department (ED) setting, limits generalizability to field settings in the United States (SOE: insufficient).

Observational studies were of very limited usefulness for informing route of administration comparisons. The four observational studies that compared different routes of naloxone administration in field settings had serious methodological shortcomings, including failure to adjust for potential confounders. ${ }^{65-68}$ In addition, the route of administration comparisons varied across the studies. No study compared IN versus IM naloxone or evaluated recently FDAapproved formulations; all studies were conducted prior to the recent FDA approvals.

Studies comparing different routes of naloxone administration were not designed to assess risk of serious injuries, such as needle stick (potentially resulting in exposure to highly potent 
synthetic opioids or infectious diseases in naloxone administering personnel). However, no cases of serious injuries were reported in the RCTs or observational studies. Evidence was insufficient to determine how comparative benefits and harms of different routes of naloxone administration differed according to demographics or clinical factors, such as the type and dose of opioid involved in overdose (including whether fentanyl or a fentanyl analogue was involved), presence of other drugs or substances, estimated time since overdose, concomitant psychiatric

comorbidities, or prior overdose episodes. There was also insufficient evidence to determine how the type or training of EMS personnel administering naloxone impacted comparisons involving different routes of administration or doses of naloxone. Most studies that provided information regarding the level of training of EMS personnel who administered naloxone reported that they were paramedics or had Advanced Life Support certification, reflecting the scope of practice for EMS personnel. Only one study comparing different routes of administration reported the type of opioid involved in suspected overdoses; ${ }^{64}$ it was conducted in Iran, with a high proportion of overdoses related to use of opium.

There was insufficient evidence to determine the optimal dose of naloxone by route of administration. No study directly compared different doses of naloxone administered via the same route. It was not possible to determine effects of dose via indirect comparisons from studies of one route of administration versus another, given the small number of studies and differences across in factors other than dose, such as the concentration of IN naloxone formulation, field versus ED setting administration of naloxone, and methodological limitations in the studies.

Evidence was too limited to reliably determine effects of hospital transport versus nontransport following successful treatment of opioid overdose. Although six studies reported low rates ( 0 to $1.2 \%$ ) of death or serious adverse events among patients who received naloxone for opioid overdose and were not transported to a health care facility, ${ }^{69-74}$ there was no comparison group of patients who were transported, and the studies provided limited details regarding the characteristics of the nontransported patients. This makes interpretation of findings difficult, as patients who refuse transport or are assessed as not requiring transport are likely to be at lower risk of opioid overdose-related complications than patients who are transported. For example, one study reported that 100 percent of patients who were not transported to an ED had a Glasgow Coma Scale Score of 14 or 15 following naloxone administration, compared with about half of patients were transported. ${ }^{69}$ In addition, the studies relied on medical examiner and hospital records to identify serious outcomes, which could underestimate event rates. The studies were not designed to evaluate outcomes such as linkage to treatment for opioid use disorder or subsequent repeat opioid overdose episodes.

No study compared titration of naloxone administered by EMS personnel until the patient resumes sufficient spontaneous respiratory effort versus until the patient regains consciousness or differences in timing of repeat naloxone dosing.

\section{Findings in Relationship to What Is Already Known}

Our findings are consistent with a systematic review that found a total of 4 deaths due to rebound opioid toxicity among 5,443 patients not transported following reversal of opioid overdose with naloxone ${ }^{75}$. We identified no previously published systematic review addressing the other Key Questions in our report. Our findings are generally consistent with the conclusions of a recent FDA meeting that focused on naloxone dosing for devices intended for use by laypeople. ${ }^{53}$ The committee convened by the FDA generally found a lack of evidence to determine optimal dosing of naloxone. ${ }^{76}$ This meeting included additional data and information 
submitted by manufacturers of naloxone. At the meeting, evidence to support higher dosing of naloxone was largely based on pharmacokinetic data and considerations regarding increased reports of overdose episodes related to high potency synthetic opioids. The current pharmacokinetic standard for new naloxone products is that they achieve levels comparable to or greater than the approved starting dose of $0.4 \mathrm{mg}$ of naloxone administered by the IV, IM, or subcutaneous (SC) routes. Clinical data suggest that lower amounts of naloxone (0.05 to $0.1 \mathrm{mg}$ ) may be sufficient to produce sufficient opioid antagonism in most cases, though the amount required may vary depending on the dose, potency, and duration of action of the opioid involved in the overdose. ${ }^{77}$ One randomized pharmacokinetic study found that the mean naloxone concentration area under the receiver operating characteristic curve was similar for a single IM/SC injection of $0.4 \mathrm{mg}$ naloxone using the recently FDA-approved auto-injector and using a standard syringe, though the maximum concentration of naloxone was 15 percent higher with administration using the autoinjector. ${ }^{59}$ A pharmacokinetic study of IN naloxone found that the plasma concentration AUROC and peak plasma concentration for a single dose of concentrated IN naloxone ( $4 \mathrm{mg} / 0.1 \mathrm{~mL}$ ) exceeded that of $0.4 \mathrm{mg}$ IM, and was similar to a $2 \mathrm{mg}$ IM dose, or the highest recommended initial dose of IM naloxone. ${ }^{78}$ Factors potentially affecting the bioavailability of IN naloxone include the volume administered per nostril (larger volumes are swallowed and not bioavailable), whether the patient is conscious or unconscious (less of the intranasal dose may be swallowed by unconscious patients), position of the patient, differences in nasal physiology (e.g. obstruction, anatomical variation), drug interactions (e.g., use of IN cocaine), and certain diseases. ${ }^{59,77}$

Clinical evidence at the FDA meeting of some relevance to this report include a study of national EMS registry data that found that the proportion of overdose episodes requiring repeat doses of naloxone increased from 14 to 18 percent from 2012 to 2015, potentially suggesting a need for higher initial doses in order to reduce the need for repeat dosing; ${ }^{50}$ this study has subsequently been published. ${ }^{79}$ Another retrospective study found that approximately one-third of cases involving administration of off-label IN naloxone ( $2 \mathrm{mg} / 2 \mathrm{~mL}$ ) by first responders in New York and New Jersey involved multiple doses. ${ }^{80}$ However, interpretation of these studies is a challenge. In both studies, it was unclear if repeat doses were administered because of failure of initial doses, changes in EMS protocols regarding repeat dosing, differences in the threshold for repeat dosing, or other factors. The studies, moreover, were not designed to assess differences in clinical outcome between patients receiving single versus multiple doses. In contrast to community settings in which patients may not receive adequate ventilatory support, in EMS administration settings the negative clinical consequences of requiring repeat naloxone dosing may be less important. One study presented at the FDA meeting found that survival rates were very similar following IN administration of one, two, or $\geq$ three doses of IN naloxone (93\% to $95 \%$ in all groups). ${ }^{56,57}$ The studies were not designed to determine the specific opioids involved in the overdose episodes.

Regarding route of administration, our finding regarding the effectiveness of higher concentration IN naloxone when administered by EMS personnel appears consistent with some data presented at the FDA meeting. The retrospective survey of first responder administration of off-label IN naloxone ( $2 \mathrm{mg} / 2 \mathrm{~mL}$ ) for suspected opioid overdose in New York and New Jersey reported high rates of survival (93\% to 97\%). A prior uncontrolled study found that ${ }^{80}$ approximately 83 percent of patients responded to IN naloxone $(2 \mathrm{mg} / 2 \mathrm{~mL})$ administered by EMS personnel. ${ }^{81}$ For the recently approved naloxone auto-injector $(0.4 \mathrm{mg})$, post-marketing surveillance data indicate very few spontaneously reported adverse events related to drug 
ineffectiveness (4 cases, or $0.0015 \%$ ); these data include cases of auto-injector use in community settings by laypeople. ${ }^{59}$

Unpublished data presented at the FDA meeting have not undergone independent peer review. In addition, some studies were funded by industry.

\section{Applicability}

Several factors limited the applicability of our findings. A key applicability limitation is that all studies meeting inclusion criteria evaluated older and/or off-label formulations of naloxone. No study evaluated the FDA-approved naloxone auto-injector for IM administration, at either a dose of $0.4 \mathrm{mg}$ or the very recently approved $2 \mathrm{mg}$ dose. Therefore, studies that involved IM naloxone were unable to assess potential benefits of the auto-injector with regard to ease of use, higher plasma concentrations compared with the same dose administered without the autoinjector, or decreased risk of needle-stick, or evaluate effects of the higher (2 mg) dose. Similarly, no study evaluated the recently FDA-approved formulations of highly-concentrated IN naloxone. One trial evaluated IN naloxone at a concentration lower $(2 \mathrm{mg} / 5 \mathrm{~mL})$ than used offlabel in the United States $(2 \mathrm{mg} / 2 \mathrm{~mL})^{62}$ and another trial evaluated IN naloxone at a concentration ( $2 \mathrm{mg} / 1 \mathrm{~mL}$ ) that appears to have been formulated specifically for that study, and is not available in any product otherwise. ${ }^{63}$ The concentration of naloxone in FDA-approved formulations ( $4 \mathrm{mg} / 0.1 \mathrm{~mL}$ or the very recently approved $2 \mathrm{mg} / 0.1 \mathrm{~mL}$ ) are substantially higher than the typical concentration used off-label for IN administration $(2 \mathrm{mg} / 2 \mathrm{~mL})$, requiring a lower volume in a system designed to deliver an accurate dose. Studies indicate very high usability rates (>90\%) with the auto-injector and IN reformulation compared with older/off-label devices, even without prior training. ${ }^{78,82}$

The settings of some studies may also limit applicability to use of naloxone in U.S. field settings by EMS personnel. All of the RCTs that compared naloxone routes of administration were conducted in non-U.S. settings (Australia and Iran), where EMS and ED management of opioid overdose, and characteristics of opioid overdose may differ from the United States. For example, in the Iranian trial, a high proportion of opioid overdoses was related to use of opium. ${ }^{64}$

Applicability was also limited by the populations evaluated in the studies. In almost all studies, characteristics of the opioid overdose, such as the specific opioid involved (including whether it was a sustained-release/long-acting opioid), opioid dose, time since overdose, concomitant substances and drugs were not reported, and subgroup analyses were not reported. In addition, almost all studies were conducted before the recent increase in availability of and exposure to illicitly manufactured high potency synthetic opioids. In studies regarding patients who received naloxone for opioid overdose who were not transported to a health care facility, details regarding the characteristics of patients were limited. This poses a challenge for interpreting the results of these studies, because patients who refuse transport or are not transported for other reasons are likely to differ substantially from patients who are transported. One study reported that 100 percent of patients who were not transported to an ED had a Glasgow Coma Scale score of 14 or 15, compared with about 50 percent of patients who were transported, but did not compare outcomes in patients transported versus those not transported. ${ }^{69}$

\section{Implications for Clinical and Policy Decisionmaking}

Although we found limited evidence to address the questions in our report, our findings have potential implications for clinical and policy decisionmaking. Regarding the route of administration, limited evidence suggests that higher concentration IN naloxone and IM 
naloxone may be similarly effective for field treatment of suspected overdose by EMS personnel. As noted previously, a factor complicating clinical and policy decisionmaking regarding the optimal route of administration is that no study included in this report has evaluated the recently FDA-approved naloxone auto-injectors or highly-concentrated formulations of IN naloxone. Assuming similar effectiveness, factors that might inform decisions regarding naloxone route of administration include ease of use, safety concerns (e.g., reduced risk of needle stick, reduced risk of agitation post-administration), and pharmacokinetics. Data indicate very high usability for both the naloxone auto-injector and reformulated IN naloxone; ${ }^{78,82}$ in fact, these products were designed to be used by laypeople with no medical training, suggesting that they could be used by basic as well as more advanced levels of EMS providers. A high level of concern regarding potential exposure of EMS personnel to infections or high potency opioids due to needle stick injuries would seem to favor needleless routes of administration such as IN naloxone, though the auto-injector is designed to provide needle stick protection to the naloxone administrator. Other factors that might impact decisions regarding the route of administration include availability and costs for different formulations/delivery systems. In 2016, the price was $\$ 4,500$ for the naloxone $0.4 \mathrm{mg}$ auto-injector increased from $\$ 690$ in 2014), \$150.00 for IN naloxone $4 \mathrm{mg} / 0.1 \mathrm{ml}$ (2 pack), \$14 to $\$ 24$ for $0.4 \mathrm{mg}$ dose of naloxone $0.4 \mathrm{mg} / \mathrm{mL}$ and $\sim 40$ for a $2 \mathrm{mg}$ dose of naloxone $(1 \mathrm{mg} / \mathrm{mL}) .{ }^{83}$ Therefore, the high costs of the new, easier-to-use formulations may represent a significant barrier to their uptake. Although IV administration of naloxone also remains an option, it appears to be on the decline, due to the need to attain IV access, which can be difficult in injection drug users and cause delays in administration. In addition, Basic Life Support EMS providers are generally not permitted to initiate IV therapy, limiting such personnel to naloxone administration via the IN or IM routes. The effective half-life of naloxone is longer for IM versus IV administration, which may be a factor in patients on high doses of opioids or those exposed to long-acting/extended-release or high potency opioids.

There was very little evidence to inform decisions regarding optimal dose of naloxone. An important current factor for informing decisions regarding naloxone dose is the increasing availability of high potency synthetic opioids, which may require higher naloxone doses to reverse. At the recent FDA meeting, the Advisory Committee voted by a slight majority to recommend an increase in the minimum dose of parenteral naloxone from $0.4 \mathrm{mg}$ in devices approved for laypeople use. ${ }^{84}$ Potential trade-off with higher doses include a higher rate of successful opioid overdose versus a higher risk and severity of withdrawal. In addition to the discomfort that withdrawal causes in patients, it can also result in agitation, with potential for injury to patients or EMS personnel, as well as vomiting with the potential for aspiration. A factor that distinguishes administration of naloxone by laypeople from administration by EMS personnel is that EMS personnel are more likely to be able to provide adequate ventilatory support in cases in which initial doses of naloxone are too low, potentially decreasing the risk of inadequately treated overdose due to lower initial doses in EMS settings. Recently, the FDA approved a 2 mg auto-injector for IM administration, which is thought to be pharmacokinetically similar to the $4 \mathrm{mg} / 0.1 \mathrm{~mL}$ IN dose. ${ }^{85}$ Even more recently, the FDA approved a $2 \mathrm{mg} / 0.1 \mathrm{~mL}$ formulation of IN naloxone, underscoring ongoing uncertainty regarding optimal dosing. Factors that could inform the use of higher dose naloxone in EMS settings is the prevalence of overdoses associated with high potency opioids or the proportion of patients requiring repeated doses.

We found no evidence with which to guide timing of repeat dosing or whether to dose to full consciousness or until having adequate respirations. The optimum timing of repeat dosing is likely to depend on the ability of EMS personnel to provide adequate ventilatory support in 
patients who do not respond to initial doses, the time required for naloxone to reach plasma concentrations sufficient to reverse overdose symptoms (which varies according to route of administration and dose), and the likelihood of precipitating acute withdrawal with repeat doses. However, EMS personnel vary with regard to their ability to provide ventilatory support, and may not have uniform access to tools to assess for adequate ventilation. With regard to whether it is necessary to dose patients until they reach full consciousness, trade-offs to consider include the likelihood of precipitating withdrawal or complications related to assisted ventilation (e.g., gastric insufflation with risk of emesis and aspiration) versus a reduction in risk of recurrence of opioid overdose symptoms or complications related to decreased level of consciousness (e.g., aspiration, upper airway obstruction). In addition, negative pressure ventilation (i.e., patients breathing on their own) is more likely to achieve adequate oxygenation than manual positive pressure ventilation. In overdoses involving opioids plus nonopioid substances and drugs, attempting to dose naloxone to achieve full consciousness may be futile and cause more severe withdrawal and agitation.

With regard to need for hospital transport following successful treatment of opioid overdose, very limited evidence suggests a low likelihood of serious adverse outcomes in patients who refuse hospital transport. It is not known whether hospital transport is associated with beneficial effects on outcomes such as linkage to treatment for opioid use disorder or decreased risk of subsequent opioid overdose episodes. It is likely that patients who weren't transported were at low risk of adverse outcomes compared to those who were transported; studies were not designed to address comparative benefits and harms of hospital transport versus nontransport in patients with similar characteristics. Factors that may impact policies regarding need for transport include whether patients are dosed until they are fully conscious, the presence of comorbidities, involvement of other substances in the overdose, likelihood of recurrence of opioid overdose symptoms (e.g., in patients exposed to long-acting opioids), the ability of EMS personnel to identify patients at high risk for recurrence for opioid overdose symptoms or other complications, and medico-legal considerations, such as mandated transport in some communities.

Policy decisions related to expansion of the scope of EMS practice for basic life support EMS personnel to include administration of naloxone could be informed by the successful use of naloxone by non-EMS first responders and laypeople. ${ }^{86-89}$ The FDA is supporting efforts to make naloxone available over the counter, though no over-the-counter naloxone preparation is currently FDA-approved. ${ }^{90}$ In a number of states, naloxone is already available without an individual prescription, through standing orders or collaborative practice agreements with prescribers. $^{91,92}$

\section{Limitations of the Systematic Review Process}

Our review had potential limitations. We limited inclusion to English language articles, which could result in language bias. However, we did not identify any foreign language studies that appeared to meet inclusion criteria in our literature searches or in reference lists. Because there were few RCTs addressing the Key Questions, we included observational studies, which are more susceptible to bias, even when designed and conducted well. The observational studies included in this report were assessed as having high risk of bias due to serious methodological shortcomings, including failure to adjust for potential confounders or failure to include a control group, and were of limited usefulness for reaching more reliable conclusions. Because of the small number of studies conducted in field settings, we modified the protocol to include studies 
performed in the ED setting; this resulted in inclusion of only one additional trial. ${ }^{64}$ Although we broadened inclusion criteria to include lay professionals (e.g., police, non-EMS firefighters, and others) administering naloxone in field settings, no additional studies in which naloxone was administered by such professionals met inclusion criteria.

We attempted to identify potentially relevant unpublished studies through searches on clinicaltrials.gov, reviewing materials presented at a recent FDA meeting on naloxone dosing, sending requests to manufacturers of naloxone for Scientific Information Packets, and contacting federal agencies that have funded naloxone research. Although we did not identify additional studies that met inclusion criteria, it is possible that some unpublished studies remained unidentified. Due to the small number of studies, clinical heterogeneity, and methodological limitations, we did not attempt meta-analysis.

\section{Limitations of the Evidence Base}

The major limitation of the evidence base is the lack of evidence to adequately address the Key Questions. Importantly, no study evaluated the recently FDA-approved auto-injectors or highly-concentrated IN reformulation. In addition, studies varied in how an adequate response to naloxone was defined, or did not define adequate response. No study evaluated health care utilization indicators and longer-term follow-up data was generally lacking. Studies of outcomes in patients who were not transported to a health care facility following successful treatment of overdose with naloxone did not have a comparison group of similar patients who were transported, making interpretation of findings a challenge. Further, these studies focused on immediate or short-term risk of serious harms such as death, and were not designed to assess other potential outcomes impacted by transport to a health care facility, such as linkage to treatment for opioid use disorder or subsequent future overdose events.

\section{Research Recommendations}

Opioid overdoses have increased dramatically, have potentially devastating consequences, and are potentially reversible. Additional research is urgently needed to optimize administration of naloxone by EMS personnel via parenteral versus needleless routes. Key areas for future research and methodological considerations include the following:

- Randomized trials in U.S. field settings that compare the FDA-approved IN formulation of highly-concentrated, low-volume naloxone versus the IM auto-injector would be very helpful for understanding the relative benefits and harms of alternative routes of administration.

- Investigators designing future RCTs on dose or route of administration might consider whether to utilize a blinded design, in order to reduce risk of bias in ascertainment of outcomes or management of opioid overdose. However, such a study design could pose ethical and logistical challenges in field settings. For example, an RCT would require an exception to informed consent, since patients would not be able to provide consent, or would need to provide consent prior to an overdose event occurring. The latter would also pose a challenge since the at-risk population includes injection drug users, who can be difficult to reach and engage.

- In addition to studies of naloxone administration by EMS personnel, studies of naloxone administration by non-EMS first responders and laypeople with limited medical training 
could also be informative for understanding optimal use of naloxone by Basic Life Support personnel.

- Studies are needed to compare effects of the FDA-approved versions of IN and IM naloxone versus non-FDA approved versions, which remain commonly used in clinical practice; a field trial in which ALS EMS providers in New York state used IN naloxone 2 $\mathrm{mg} / 2 \mathrm{~mL}$ and $4 \mathrm{mg} / 0.1 \mathrm{~mL}$ on alternating weeks is ongoing. ${ }^{93}$

- Studies that compare different doses (e.g., 0.4 vs. $2 \mathrm{mg}$ doses of the IM auto-injector) are needed to determine the optimal dose for various routes of administration.

- Ideally, studies would include (to the extent possible) information regarding the opioids involved in the overdoses and other patient factors, and perform subgroup analyses to evaluate for potential differential effects based on these factors. Of particular interest is whether overdoses related to illicit fentanyl or fentanyl analogues require higher doses of naloxone.

- $\quad$ Studies should also include information regarding the type of EMS personnel administering naloxone, in order to assess whether the level of training impacts benefits and harms or whether effects are similar across different levels of EMS training.

- Studies should evaluate benefits as well as important harms, including withdrawal, agitation, and injury (to the patient or EMS personnel), as well as risk of exposure of EMS personnel to high potency opioids, in order to provide balanced assessments of the trade-offs involved.

- Although IN and IM administration appears to be of greatest interest currently, studies could also address comparisons involving IV or SC administration (in some cases whether someone receives an IM or SC injection may not be clear since this depends on the length of the needle and the thickness of the subcutaneous layer in the patient receiving the injection), for which data are extremely sparse.

- Future research could leverage existing EMS data registries with naloxone administration data, which are available from a number of local and state agencies. In addition, the NHTSA funded National Emergency Medical Services Information System (NEMSIS) contains data from EMS agencies across the United States. ${ }^{94}$

- Although current registries provide important descriptive and epidemiological information regarding naloxone administration by EMS personnel, they were not designed to address the comparative effectiveness questions in this review. For example, although a study of national EMS registry data that found that the proportion of overdose episodes requiring repeat doses of naloxone increased from 14 to 18 percent from 2012 to 2015, it was not designed to determine the optimal interval for repeat dosing or if the observed increase was related to greater risk of failure of initial naloxone dosing, variability in thresholds for repeat dosing, changes in dosing protocols, or other factors. ${ }^{50}$ Ideally, registry studies should include information about the dose, formulation, and route of administration of naloxone; opioid involved in exposure; training of EMS personnel administering naloxone; clinical response to initial and repeat dosing; protocol for initial and repeat naloxone dosing; and clinical outcomes, including response rates using predefined criteria, risk for recurrence of opioid overdose symptoms, and adverse outcomes.

- Importantly, registry and other observational studies should utilize methods to reduce risk of confounding and bias, including statistical adjustment or matching. For example, studies on clinical outcomes associated with single versus multiple doses of naloxone 
should account for severity of overdose symptoms prior to initial naloxone administration to reduce confounding by indication.

- Research is needed to determine optimal timing of repeat dosing and strength of dose(s) as well as whether to dose until fully conscious or until patients have adequate respirations (e.g., in situations in which adequate ventilatory support is not available). For studies addressing either of these questions, the protocols used for naloxone dosing will need to be clearly defined, including indications for additional "rescue" dosing. Registry and pilot studies would be helpful for informing appropriate naloxone dosing protocols, to aid in the design of future clinical trials.

- For comparing effects of nontransport following successful treatment of opioid overdose with naloxone, RCTs may not be logistically or ethically feasible. However, comparative observational studies would better inform this question than the noncomparative studies currently available. For example, studies could identify patients who are not transported to a hospital and match them with patients who are transported, based on factors such as age, sex, suspected opioid involved in overdose, response to naloxone (e.g., based on Glasgow Coma Scale score), other substances and drugs involved in overdose, or other factors. Studies should supplement use of medical examiner and hospital records to identify outcomes with formal followup assessments, and evaluate outcomes such as linkage to treatment for opioid use disorder and risk of future overdose episodes, in addition to serious adverse outcomes associated with the current overdose episode, such as death and any serious complications.

- Future research could address alternative routes of administration, such as needleless administration via mask nebulization, ${ }^{95}$ sublingually, or buccally. ${ }^{21}$ An important drawback of nebulized naloxone is potential ineffectiveness if the subject is not breathing. Potential challenges in sublingual and buccal administration are that they require access to the mouth and could be impacted by vomiting or swallowing; sublingual administration in addition requires positioning under the tongue, which may be difficult. ${ }^{21}$ Endotracheal administration is a needleless route that is no longer recommended, ${ }^{96}$ since the intubated patient should be receiving adequate ventilatory support and naloxone administration with successful reversal of overdose could result in severe agitation; furthermore, endotracheal absorption is inconsistent.

- Given the changing epidemiology of opioid overdoses, particularly with regard to overdoses related to fentanyl and related substances, future systematic reviews on this topic may be warranted in the near future to incorporate new studies. 


\section{Conclusions}

Low-strength evidence suggested that intranasal (IN) naloxone at a dose of $2 \mathrm{mg}$ and concentration of $2 \mathrm{mg} / 1 \mathrm{~mL}$ is similar in efficacy to intramuscular (IM) naloxone at a dose of 2 mg, with no difference in adverse events. Research is needed on the comparative effectiveness of the U.S. Food and Drug Administration-approved naloxone auto-injectors and highlyconcentrated IN naloxone formulations, different doses, and dosing strategies. Uncontrolled studies suggest that nontransport of patients following successful naloxone reversal of overdose is associated with a low rate of serious harms, but patients were probably at low risk for such events, and no study evaluated risk of transport versus nontransport. 


\section{References}

1. Macrae J, Hyde P, Slavitt A. HHS launches multi-pronged effort to combat opioid abuse Washington, DC: U.S. Department of Health \& Human Services; July 27, 2015. http://www.hhs.gov/blog/2015/07/27/ hhs-launches-multi-pronged-effort-combatopioid-abuse.html. Accessed August 29, 2016.

2. Centers for Disease Control and Prevention. Vital signs: overdoses of prescription opioid pain relievers---United States, 1999--2008. FIGURE 2. Rates of opioid pain reliever (OPR) overdose death, OPR treatment admissions, and kilograms of OPR sold --United States, 1999--2010. MMWR Morb Mortal Wkly Rep. 2011 Nov 4;60(43):148792. PMID: 22048730.

3. Rudd RA, Seth P, David F, et al. Increases in drug and opioid overdose deaths--United States, 2010-2015. MMWR Morb Mortal Wkly Rep. 2016;65(50-51):1445-52.

4. National Center for Health Statistics. Drugpoisoning deaths involving opioid analgesics: United States, 1999-2011. NCHS Data Brief 166. Atlanta, GA: Centers for Disease Control and Prevention; September

2014. https://www.cdc.gov/nchs/products/da tabriefs/db166.htm. Accessed May 25, 2016.

5. Angst MS, Lazzeroni LC, Phillips NG, et al. Aversive and reinforcing opioid effects: a pharmacogenomic twin study. Anesthesiology. 2012 Jul;117(1):22-37. doi: 10.1097/ALN.0b013e31825a2a4e. PMID: 22713632.

6. Bohnert AS, Valenstein M, Bair MJ, et al. Association between opioid prescribing patterns and opioid overdose-related deaths. JAMA. 2011 Apr 6;305(13):1315-21. doi: 10.1001/jama.2011.370. PMID: 21467284.

7. Darke S, Hall W. Heroin overdose: research and evidence-based intervention. J Urban Health. 2003 Jun;80(2):189-200. doi: 10.1093/jurban/jtg022. PMID: 12791795.

8. Dilokthornsakul P, Moore G, Campbell JD, et al. Risk factors of prescription opioid overdose among Colorado Medicaid beneficiaries. J Pain. 2016 Apr;17(4):43643. doi: 10.1016/j.jpain.2015.12.006. PMID: 26721613.
9.

Hall AJ, Logan JE, Toblin RL, et al. Patterns of abuse among unintentional pharmaceutical overdose fatalities. JAMA. 2008 Dec 10;300(22):2613-20. doi: 10.1001/jama.2008.802. PMID: 19066381.

10. Zedler B, Xie L, Wang L, et al. Risk factors for serious prescription opioid-related toxicity or overdose among Veterans Health Administration patients. Pain Med. 2014 Nov;15(11):1911-29. doi: 10.1111/pme.12480. PMID: 24931395.

11. Schumann H, Erickson T, Thompson TM, et al. Fentanyl epidemic in Chicago, Illinois and surrounding Cook County. Clin Toxicol. 2008 Jul;46(6):501-6. doi: 10.1080/15563650701877374. PMID: 18584361.

12. Frank RG, Pollack HA. Addressing the fentanyl threat to public health. N Engl J Med. 2017 Feb 16;376(7):605-7. doi: 10.1056/NEJMp1615145. PMID: 28199808.

13. Tomassoni AJ, Hawk KF, Jubanyik K, et al. Multiple fentanyl overdoses - New Haven, Connecticut, June 23, 2016. MMWR Morb Mortal Wkly Rep. 2017 Feb 03;66(4):10711. doi: $10.15585 / \mathrm{mm} 6604 a 4$. PMID: 28151928.

14. Somerville NJ, O'Donnell J, Gladden RM, et al. Characteristics of fentanyl overdose Massachusetts, 2014-2016. MMWR Morb Mortal Wkly Rep. 2017 Apr 14;66(14):3826. doi: 10.15585/mmwr.mm6614a2. PMID: 28406883.

15. Sutter ME, Gerona RR, Davis MT, et al. Fatal fentanyl: One pill can kill. Acad Emerg Med. 2016 Jun 20doi: 10.1111/acem.13034. PMID: 27322591.

16. Bailey JE, Campagna E, Dart RC, et al. The underrecognized toll of prescription opioid abuse on young children. Ann Emerg Med. 2009 Apr;53(4):419-24. doi: 10.1016/j.annemergmed.2008.07.015. PMID: 18774623.

17. Bond GR, Woodward RW, Ho M. The growing impact of pediatric pharmaceutical poisoning. J Pediatr. 2012 Feb;160(2):26570 e1. doi: 10.1016/j.jpeds.2011.07.042. PMID: 21920539. 
18. Darke S, Mattick RP, Degenhardt L. The ratio of non-fatal to fatal heroin overdose. Addiction. 2003 Aug;98(8):1169-71. PMID: 12873254.

19. Dunn KM, Saunders KW, Rutter CM, et al. Opioid prescriptions for chronic pain and overdose: a cohort study. Ann Intern Med. 2010 Jan 19;152(2):85-92. doi: 10.7326/0003-4819-152-2-20100119000006. PMID: 20083827.

20. Boyer EW. Management of opioid analgesic overdose. N Engl J Med. 2012 Jul 12;367(2):146-55. doi: 10.1056/NEJMra1202561. PMID: 22784117.

21. Strang J, McDonald R, Alqurshi A, et al. Naloxone without the needle - systematic review of candidate routes for non-injectable naloxone for opioid overdose reversal. Drug Alcohol Depend. 2016 Jun 1;163:16-23. doi: 10.1016/j.drugalcdep.2016.02.042. PMID: 26996745.

22. FDA approves new hand-held auto-injector to reverse opioid overdose. FDA news release, April 3, 2014. Silver Spring, MD: U.S. Food and Drug Administration; 2014. http://www.fda.gov/NewsEvents/New sroom/PressAnnouncements/ucm391465.ht m. Accessed February 15, 2017.

23. FDA moves quickly to approve easy-to-use nasal spray to treat opioid overdose. FDA news release, Nov. 18, 2015. Silver Spring, MD: U.S. Food and Drug Administration; 2015. http://www.fda.gov/NewsEvents/New sroom/PressAnnouncements/ucm473505.ht m. Accessed August 29, 2016.

24. Mueller SR, Walley AY, Calcaterra SL, et al. A review of opioid overdose prevention and naloxone prescribing: implications for translating community programming into clinical practice. Subst Abus.

2015;36(2):240-53. doi:

10.1080/08897077.2015.1010032. PMID: 25774771.

25. Walley AY, Xuan Z, Hackman HH, et al. Opioid overdose rates and implementation of overdose education and nasal naloxone distribution in Massachusetts: interrupted time series analysis. BMJ. 2013;346:f174. doi: 10.1136/bmj.f174. PMID: 23372174.
26. Wheeler E, Jones TS, Gilbert MK, et al. Opioid overdose prevention programs providing naloxone to laypersons - United States, 2014. MMWR Morb Mortal Wkly Rep. 2015 Jun 19;64(23):631-5. PMID: 26086633.

27. Kim D, Irwin KS, Khoshnood K. Expanded access to naloxone: options for critical response to the epidemic of opioid overdose mortality. Am J Public Health. 2009 Mar;99(3):402-7. doi: 10.2105/AJPH.2008.136937. PMID: 19150908.

28. Wermeling DP. Review of naloxone safety for opioid overdose: practical considerations for new technology and expanded public access. Ther Adv Drug Saf. 2015 Feb;6(1):20-31. doi: 10.1177/2042098614564776. PMID: 25642320.

29. Clarke SF, Dargan PI, Jones AL. Naloxone in opioid poisoning: walking the tightrope. Emerg Med J. 2005 Sep;22(9):612-6. PMID: 16113176.

30. Buajordet I, Naess AC, Jacobsen D, et al. Adverse events after naloxone treatment of episodes of suspected acute opioid overdose. Eur J Emerg Med. 2004 Feb;11(1):19-23. PMID: 15167188.

31. EMS1. 5 things EMS providers need to know about opioid overdose and respiratory compromise.

2016. http://www.ems1.com/emsproducts/Capnography/articles/47753048-5things-EMS-providers-need-to-know-aboutopioid-overdose-and-respiratorycompromise/. Accessed October 7, 2016.

32. Alcorn T. America embraces treatment for opioid drug overdose. Lancet. 2014 Jun 7;383(9933):1957-8. PMID: 24936622.

33. Fala L, Welz JA. New perspectives in the treatment of opioid-induced respiratory depression. Am Health Drug Benefits. 2015 Oct;8(6 Suppl 3):S51-63. PMID: 26629261.

34. National EMS Information System (NEMSIS). Create a report (NEMSIS Cube). 2017. http://nemsis.org/reportingTools/repor ts/nationalReports/createAReport.html. Accessed Februrary 15, 2017. 
35. National Highway Traffic Safety

Administration. The national EMS scope of practice model. DOT HS 810657.

Washington, DC: U.S. Department of Transportation; February

2007. http://www.ems.gov/education/EMSS cope.pdf Accessed August 29, 2016.

36. Davis CS, Southwell JK, Niehaus VR, et al. Emergency medical services naloxone access: a national systematic legal review. Acad Emerg Med. 2014 Oct;21(10):1173-7. doi: 10.1111/acem.12485. PMID: 25308142.

37. Faul M, Dailey MW, Sugerman DE, et al. Disparity in naloxone administration by emergency medical service providers and the burden of drug overdose in US rural communities. Am J Public Health. 2015 Jul;105 Suppl 3:e26-32. doi: 10.2105/AJPH.2014.302520. PMID: 25905856.

38. Weiner SG, Mitchell PM, Temin ES, et al. Use of intranasal naloxone by basic life support providers. Prehosp Emerg Care. 2017:1-5. doi:

10.1080/10903127.2017.1282562.

39. Lavonas EJ, Drennan IR, Gabrielli A, et al. Part 10: Special circumstances of resuscitation: 2015 American Heart Association guidelines update for cardiopulmonary resuscitation and emergency cardiovascular care. Circulation. 2015 Nov 3;132(18 Suppl 2):S501-18. doi: 10.1161/CIR.0000000000000264. PMID: 26472998.

40. National Association of State EMS Officials. National model EMS clinical guidelines.

2014. https://www.nasemso.org/Projects/Mo delEMSClinicalGuidelines/documents/Natio nal-Model-EMS-Clinical-Guidelines230ct2014.pdf. Accessed August 29, 2016.

41. World Health Organization (WHO). Community management of opioid overdose. Geneva, Switzerland; 2014. http://www.who.int/substance_abuse/ publications/management_opioid_overdose/ en/.
42. Connors NJ, Nelson LS. The evolution of recommended naloxone dosing for opioid overdose by medical specialty. J Med Toxicol. 2016 Sep;12(3):276-81. doi: 10.1007/s13181-016-0559-3. PMID: 27271032.

43. EVZIO (naloxone $\mathrm{HCl}$ injection) $2 \mathrm{mg}$ autoinjector Intelligent Voice Guidance ${ }^{\mathrm{TM}}$.

Reach for voice guidance. https://evzio.com/patient/aboutevzio/index.php. Accessed February 15, 2017.

44. Strang J, McDonald R, Tas B, et al. Clinical provision of improvised nasal naloxone without experimental testing and without regulatory approval: imaginative shortcut or dangerous bypass of essential safety procedures? Addiction. 2016 Apr;111(4):574-82. doi: 10.1111/add.13209. PMID: 26840916.

45. Morrone WR. President's message: Food and Drug Administration approved naloxone and continued use of improvised nasal naloxone: What is a treatment advocate and educator to do? J Addict Dis. 2016 OctDec;35(4):339-45. doi: 10.1080/10550887.2016.1226582. PMID: 27731792.

46. Traynor K. FDA approves first intranasal naloxone product. Am J Health Syst Pharm. 2016;73(1):e2-e3. doi: 10.2146/news160002. PMID: 26683675

47. ADAPT Pharma Operations Limited. NARCAN $^{\mathrm{R}}$ (naloxone $\mathrm{HCl}$ ) nasal spray $2 \mathrm{mg}$ approved y U.S. Food and Drug Administration (FDA).

2016. http://adaptpharma.com/adapt_press_r elease/january-25-2017-narcan-nasal-spray2mg-approved-by-us-food-and-drugadministration/. Accessed Februrary 15, 2017.

48. INVDIVIOR. Indivior feceives complete response letter from FDA not approving naloxone nasal spray new drug application for opioid overdose.

2015. http://www.indivior.com/wpcontent/uploads/2015/11/Nasal-NaloxoneFinal-Release_112415.pdf Accessed January 23, 2017. 
49. CDC Health Alert Network. Influx of fentanyl-laced counterfeit pills and fentanylrelated compounds further increases risk of fenanyl-related overdose and fatalities. 2016. https://emergency.cdc.gov/han/han003 95.asp. Accessed October 7, 2016.

50. Faul M. Trends in multiple naloxone administrations among EMS personnel: FDA Anesthetic and Analgesic Drug Products Advisory Committee and the Drug Safety and Risk Management Advisory Committee.

2016. http://www.fda.gov/downloads/Advis oryCommittees/CommitteesMeetingMaterial s/Drugs/AnestheticAndAnalgesicDrugProdu ctsAdvisoryCommittee/UCM524338.pdf Accessed January 20, 2017.

51. Millin MG, Brown LH, Schwartz B. EMS provider determinations of necessity for transport and reimbursement for EMS response, medical care, and transport: combined resource document for the National Association of EMS Physicians position statements. Prehosp Emerg Care. 2011 Oct-Dec;15(4):562-9. doi: 10.3109/10903127.2011.598625. PMID: 21797787.

52. Agency for Healthcare Research and Quality. Methods guide for effectiveness and comparative effectiveness reviews. 2014. www.effectivehealthcare.ahrq.gov. Accessed January 23, 2017.

53. U.S. Food and Drug Administration. 2016 Meeting Materials, Anesthetic and Analgesic Drug Products Advisory Committee.

2016. http://www.fda.gov/AdvisoryCommitt ees/CommitteesMeetingMaterials/Drugs/An estheticAndAnalgesicDrugProductsAdvisor yCommittee/ucm486848.htm.

54. Adapt Pharma Operations Limited. FDA Advisory Committee on the most appropriate dose or doses of naloxone to reverse the effects of life-threatening opiod overdose in the community settings.

2016. http://www.fda.gov/downloads/Advis oryCommittees/CommitteesMeetingMaterial s/Drugs/AnestheticAndAnalgesicDrugProdu ctsAdvisoryCommittee/UCM522688.pdf.
55. U.S. Food and Drug Administration. Joint meeting of anesthetic and analgesic drug products advisory committee and drug safety and risk management advisory committee.

2016. http://www.fda.gov/downloads/Advis oryCommittees/CommitteesMeetingMaterial s/Drugs/AnestheticAndAnalgesicDrugProdu ctsAdvisoryCommittee/UCM522687.pdf.

56. Amphastar Pharmaceuticals. FDA Advisory Committee Briefing Materials. Intranasal naloxone intended for use in the community. Joint meeting of the anesthetic and analgesic drug products advisory committee and the drug safety and risk management advisory committee.

2016. http://www.fda.gov/downloads/Advis oryCommittees/CommitteesMeetingMaterial s/Drugs/AnestheticAndAnalgesicDrugProdu ctsAdvisoryCommittee/UCM522689.pdf.

57. Amphastar Pharmaceuticals. Naloxone meeting: Joint meeting of the anesthetic and alagesic drug products advisory committee and the drug safety and risk management advisory committee.

2016. http://www.fda.gov/downloads/Advis oryCommittees/CommitteesMeetingMaterial s/Drugs/AnestheticAndAnalgesicDrugProdu ctsAdvisoryCommittee/UCM524334.pdf.

58. Insys Development Company Inc. Naloxone for treatment of opioide overdose 2016. http://www.fda.gov/downloads/Advis oryCommittees/CommitteesMeetingMaterial s/Drugs/AnestheticAndAnalgesicDrugProdu ctsAdvisoryCommittee/UCM522690.pdf Accessed December 19, 2016.

59. Kaleo Inc. Joint meeting of the anesthetic and analgesic drug products and the drug safety and risk management advisory committees.

2016. http://www.fda.gov/downloads/Advis oryCommittees/CommitteesMeetingMaterial s/Drugs/AnestheticAndAnalgesicDrugProdu ctsAdvisoryCommittee/UCM522691.pdf. 
60. McDonagh M, Peterson K, Raina P, et al. Avoiding bias in selecting studies. Methods guide for comparative effectiveness reviews. (Prepared by the Oregon Health \& Science University, McMaster University, and Southern California Evidence-based Practice Centers under Contract No. 290-200710057-I.) AHRQ Publication No. 13EHC045-EF. Rockville, MD: Agency for Healthcare Research and Quality; 2013. www.effectivehealthcare.ahrq.gov/rep orts/final.cfm Accessed August 29, 2016.

61. U.S. Preventive Services Task Force. U.S. Preventive Services Task Force procedure manual.

2008. http://www.uspreventiveservicestaskf orce.org/uspstf08/methods/procmanual.htm.

62. Kelly AM, Kerr D, Dietze P, et al. Randomised trial of intranasal versus intramuscular naloxone in prehospital treatment for suspected opioid overdose. Med J Aust. 2005 Jan 3;182(1):24-7. PMID: 15651944.

63. Kerr D, Kelly AM, Dietze P, et al. Randomized controlled trial comparing the effectiveness and safety of intranasal and intramuscular naloxone for the treatment of suspected heroin overdose. Addiction. 2009 Dec;104(12):2067-74. doi: 10.1111/j.13600443.2009.02724.x. PMID: 19922572.

64. Sabzghabaee AM, Eizadi-Mood N, Yaraghi A, et al. Naloxone therapy in opioid overdose patients: intranasal or intravenous? a randomized clinical trial. Arch Med Sci. 2014 May 12;10(2):309-14. doi: 10.5114/aoms.2014.42584. PMID: 24904666.

65. Merlin MA, Saybolt M, Kapitanyan R, et al. Intranasal naloxone delivery is an alternative to intravenous naloxone for opioid overdoses. Am J Emerg Med. 2010 Mar;28(3):296-303. doi: 10.1016/j.ajem.2008.12.009. PMID: 20223386.

66. Robertson TM, Hendey GW, Stroh G, et al. Intranasal naloxone is a viable alternative to intravenous naloxone for prehospital narcotic overdose. Prehosp Emerg Care. 2009 Oct-Dec;13(4):512-5. doi: 10.1080/10903120903144866. PMID: 19731165.
67. Sporer KA, Firestone J, Isaacs SM. Out-ofhospital treatment of opioid overdoses in an urban setting. Acad Emerg Med. 1996 Jul;3(7):660-7. PMID: 8816181.

68. Wanger K, Brough L, Macmillan I, et al. Intravenous vs subcutaneous naloxone for out-of-hospital management of presumed opioid overdose. Acad Emerg Med. 1998 Apr;5(4):293-9. PMID: 9562190.

69. Boyd JJ, Kuisma MJ, Alaspaa AO, et al. Recurrent opioid toxicity after pre-hospital care of presumed heroin overdose patients. Acta Anaesthesiol Scand. 2006 Nov;50(10):1266-70. PMID: 17067327.

70. Rudolph SS, Jehu G, Nielsen SL, et al. Prehospital treatment of opioid overdose in Copenhagen--is it safe to discharge onscene? Resuscitation. 2011 Nov;82(11):1414-8. doi: 10.1016/j.resuscitation.2011.06.027. PMID: 21745532.

71. Vilke GM, Buchanan J, Dunford JV, et al. Are heroin overdose deaths related to patient release after prehospital treatment with naloxone? Prehosp Emerg Care. 1999 JulSep;3(3):183-6. PMID: 10424852.

72. Vilke GM, Sloane C, Smith AM, et al. Assessment for deaths in out-of-hospital heroin overdose patients treated with naloxone who refuse transport. Acad Emerg Med. 2003 Aug;10(8):893-6. PMID: 12896894.

73. Wampler DA, Molina DK, McManus J, et al. No deaths associated with patient refusal of transport after naloxone-reversed opioid overdose. Prehosp Emerg Care. 2011 JulSep;15(3):320-4. doi: 10.3109/10903127.2011.569854. PMID: 21612385.

74. Levine M, Sanko S, Eckstein M. Assessing the risk of prehospital administration of naloxone with subsequent refusal of care. Prehosp Emerg Care. 2016;20(5):566-9. PMID: 27018626.

75. Willman MW, Liss DB, Schwarz ES, et al. Do heroin overdose patients require observation after receiving naloxone? Clin Toxicol (Phila). 2017 Feb;55(2):81-7. doi: 10.1080/15563650.2016.1253846. PMID: 27849133. 
76. Fiore K. FDA splits on naloxone dose. 2016. http://www.medpagetoday.com/Neuro logy/GeneralNeurology/60632.

77. Dowling J, Isbister GK, Kirkpatrick CM, et al. Population pharmacokinetics of intravenous, intramuscular, and intranasal naloxone in human volunteers. Ther Drug Monit. 2008 Aug;30(4):490-6. doi: 10.1097/FTD.0b013e3181816214. PMID: 18641540 .

78. Krieter P, Chiang N, Gyaw S, et al. Pharmacokinetic properties and human use characteristics of an FDA-approved intranasal naloxone product for the treatment of opioid overdose. J Clin Pharmacol. 2016 Oct;56(10):1243-53. doi: 10.1002/jcph.759. PMID: 27145977.

79. Faul M, Lurie P, Kinsman J, et al. Multiple naloxone administrations among EMS Providers is increasing. Prehosp Emerg Care. 2017(8):1-8. doi: 10.1080/10903127.2017.1315203. PMID: 28481656.

80. New York State Public Safety. Retrospective analysis of data from NY and NJ. Amphastar

Pharmaceuticals. http://www.fda.gov/downl oads/AdvisoryCommittees/CommitteesMeet ingMaterials/Drugs/AnestheticAndAnalgesi cDrugProductsAdvisoryCommittee/UCM52 2689.pdf. Accessed January 23, 2017. p. 26.

81. Barton ED, Colwell CB, Wolfe T, et al. Efficacy of intranasal naloxone as a needleless alternative for treatment of opioid overdose in the prehospital setting. J Emerg Med. 2005 Oct;29(3):265-71. PMID: 16183444.

82. Edwards ET, Edwards ES, Davis E, et al. Comparative usability study of a novel autoinjector and an intranasal system for naloxone delivery. Pain Ther. 2015 Jun;4(1):89-105. doi: 10.1007/s40122-0150035-9. PMID: 25910473.

83. Gupta R, Shah ND, Ross JS. The rising price of naloxone - risks to efforts to stem overdose deaths. N Engl J Med. 2016 Dec 08;375(23):2213-5. doi: 10.1056/NEJMp1609578. PMID: 27959694.
84. Leonard K. Major changes could be coming to overdose-reversal drugs. U.S. News \& World Report:

2016. http://www.usnews.com/news/articles/ 2016-10-06/fda-panel-more-naloxoneneeded-in-overdose-reversal-device Accessed January 23, 2017.

85. Department of Health and Human Services. NDA 209862 approval.

2016. http://www.accessdata.fda.gov/drugsa tfda docs/appletter/2016/209862Orig1s000lt r.pdf Accessed January 23, 2017.

86. Rando J, Broering D, Olson JE, et al. Intranasal naloxone administration by police first responders is associated with decreased opioid overdose deaths. Am J Emerg Med. 2015 Sep;33(9):1201-4. doi: 10.1016/j.ajem.2015.05.022. PMID: 26095132.

87. Fisher R, O'Donnell D, Ray B, et al. Police officers can safely and effectively administer intranasal naloxone. Prehosp Emerg Care. 2016;20(6):675-80. doi: 10.1080/10903127.2016.1182605. PMID: 27218446.

88. Davis C. Naloxone for community opiod overdose reversal. http://prescribetoprevent.org/wp20 15/wpcontent/uploads/PHLRKnowledgeAsset_Nal oxone_FINALfull_8June15.pdf Accessed February 15, 2017.

89. Giglio RE, Li G, DiMaggio CJ. Effectiveness of bystander naloxone administration and overdose education programs: a meta-analysis. Inj Epidemiol. 2015;2(1):10. doi: 10.1186/s40621-0150041-8. PMID: 27747742.

90. Mahoney K. FDA supports greater access to naloxone to help reduce opioid overdose deaths.

2016. http://blogs.fda.gov/fdavoice/index.ph p/2016/08/fda-supports-greater-access-tonaloxone-to-help-reduce-opioid-overdosedeaths/. Accessed February 15, 2017.

91. Brennan Z. OTC opioid overdose antidote: why is it not FDA approved? ; 2016. http://www.raps.org/RegulatoryFocus/News/2016/02/24/24400/OTCOpioid-Overdose-Antidote-Why-is-it-notFDA-Approved/. Accessed February 15, 2017. 
92. Davis CS, Carr D. Legal changes to increase access to naloxone for opioid overdose reversal in the United States. Drug \& Alcohol Dependence. 2015 Dec 1;157:11220. doi: 10.1016/j.drugalcdep.2015.10.013. PMID: 26507172.

93. Dailey M. Report of a Field Trial for Comparason of Two Intranasal Naloxnoe Delivery Devices: An Equipoise Trial. Society of Academic Emergency Medicine; 2017 Friday, May 19; Hyatt Regency, Orlando.

94. National EMS Information System (NEMSIS). The project. 2005. http://nemsis.org/theProject/index.htm l. Accessed February 15, 2017.
95. Weber JM, Tataris KL, Hoffman JD, et al. Can nebulized naloxone be used safely and effectively by emergency medical services for suspected opioid overdose? Prehosp Emerg Care. 2012 Apr-Jun;16(2):289-92. doi: 10.3109/10903127.2011.640763. PMID: 22191727.

96. Callaway CW, Soar J, Aikiki M, et al. Part 4: advanced life support 2015 international consensus on cardiopulmonary resuscitation and emergency cardiovascular care science with treatment recommendations. Cardiac arrest in special circumstances.

2015. http://circ.ahajournals.org/content/132 $/ 16$ suppl $1 /$ S84\#sec-173. Accessed February 15, 2017. 


\section{Appendix A. Search Strategies}

Database: Ovid MEDLINE(R)

1 overdose*.mp.

2 drug overdose/ or opioid-related disorders/

3 Naloxone/ or naloxone.mp.

4 (1 or 2$)$ and 3

Database: PsychINFO

1 naloxone.mp. or exp NALOXONE/

2 exp DRUG OVERDOSES/

3 overdos*.mp. [mp=title, abstract, heading word, table of contents, key concepts, original title, tests \& measures]

$4 \quad 1$ and (2 or 3$)$

Database: EBM Reviews - Cochrane Central Register of Controlled Trials

1 naloxone.mp. or exp NALOXONE/

2 exp DRUG OVERDOSES/

3 overdos*.mp. [mp=title, original title, abstract, mesh headings, heading words, keyword]

41 and (2 or 3$)$

Database: CINAHL

((MH "Naloxone") OR "naloxone") AND ((MH "Overdose") OR "overdose" OR overdos*) 


\section{Appendix B. Included Studies List}

1. Boyd JJ, Kuisma MJ, Alaspaa AO, et al. Recurrent opioid toxicity after pre-hospital care of presumed heroin overdose patients. Acta Anaesthesiol Scand. 2006 Nov;50(10):1266-70. PMID: 17067327.

2. Kelly AM, Kerr D, Dietze P, et al. Randomised trial of intranasal versus intramuscular naloxone in prehospital treatment for suspected opioid overdose. Med J Aust. 2005 Jan 3;182(1):24-7. PMID: 15651944.

3. Kerr D, Kelly AM, Dietze P, et al. Randomized controlled trial comparing the effectiveness and safety of intranasal and intramuscular naloxone for the treatment of suspected heroin overdose. Addiction. 2009 Dec;104(12):2067-74. doi: 10.1111/j.1360-0443.2009.02724.x. PMID: 19922572.

4. Levine M, Sanko S, Eckstein M. Assessing the risk of prehospital administration of naloxone with subsequent refusal of care. Prehosp Emerg Care. 2016;20(5):566-9. PMID: 27018626

5. Merlin MA, Saybolt M, Kapitanyan $\mathrm{R}$, et al. Intranasal naloxone delivery is an alternative to intravenous naloxone for opioid overdoses. Am J Emerg Med. 2010 Mar;28(3):296303. doi: 10.1016/j.ajem.2008.12.009. PMID: 20223386.
6. Robertson TM, Hendey GW, Stroh $\mathrm{G}$, et al. Intranasal naloxone is a viable alternative to intravenous naloxone for prehospital narcotic overdose. Prehosp Emerg Care. 2009 Oct-Dec;13(4):512-5. doi: 10.1080/10903120903144866. PMID: 19731165.

7. Rudolph SS, Jehu G, Nielsen SL, et al. Prehospital treatment of opioid overdose in Copenhagen--is it safe to discharge on-scene? Resuscitation. 2011 Nov;82(11):1414-8. doi: 10.1016/j.resuscitation.2011.06.027. PMID: 21745532. Excluded for wrong comparator.

8. Sabzghabaee AM, Eizadi-Mood N, Yaraghi A, et al. Naloxone therapy in opioid overdose patients: intranasal or intravenous? a randomized clinical trial. Arch Med Sci. 2014 May 12;10(2):309-14. doi: 10.5114/aoms.2014.42584. PMID: 24904666.

9. Sporer KA, Firestone J, Isaacs SM. Out-of-hospital treatment of opioid overdoses in an urban setting. Acad Emerg Med. 1996 Jul;3(7):660-7. PMID: 8816181.

10. Vilke GM, Buchanan J, Dunford JV, et al. Are heroin overdose deaths related to patient release after prehospital treatment with naloxone? Prehosp Emerg Care. 1999 JulSep;3(3):183-6. PMID: 10424852.

11. Vilke GM, Sloane C, Smith AM, et al. Assessment for deaths in out-ofhospital heroin overdose patients treated with naloxone who refuse transport. Acad Emerg Med. 2003 Aug;10(8):893-6. PMID: 12896894. 
12. Wampler DA, Molina DK, McManus J, et al. No deaths associated with patient refusal of transport after naloxone-reversed opioid overdose. Prehosp Emerg Care. 2011 Jul-Sep;15(3):320-4. doi: 10.3109/10903127.2011.569854.

PMID: 21612385.

13. Wanger K, Brough L, Macmillan I, et al. Intravenous vs subcutaneous naloxone for out-of-hospital management of presumed opioid overdose. Acad Emerg Med. 1998 Apr;5(4):293-9. PMID: 9562190. 


\section{Appendix C. Excluded Studies List}

1. Alexander JL, Burton JH, Bradshaw JR, et al. Suspected opioid-related emergency medical services encounters in a rural state, 19972002. Prehosp Emerg Care. 2004 Oct-Dec;8(4):427-30. PMID: 15626007. Excluded for wrong outcome.

2. Ashton H, Hassan Z. Best evidence topic report. Intranasal naloxone in suspected opioid overdose. Emerg Med J. 2006 Mar;23(3):221-3. PMID: 16498165. Excluded; not a study.

3. Baca CT, Grant KJ. Take-home naloxone to reduce heroin death. Addiction. 2005 Dec;100(12):182331. PMID: 16367983. Excluded for wrong population.

4. Barton ED, Colwell CB, Wolfe T, et al. Efficacy of intranasal naloxone as a needleless alternative for treatment of opioid overdose in the prehospital setting. J Emerg Med. 2005

Oct;29(3):265-71. PMID:

16183444. Excluded for wrong comparator.

5. Barton ED, Ramos J, Colwell C, et al. Intranasal administration of naloxone by paramedics. Prehosp Emerg Care. 2002 Jan-Mar;6(1):548. PMID: 11789651. Excluded for wrong comparator.
6. Bird SM, McAuley A, Perry S, et al. Effectiveness of Scotland's national naloxone programme for reducing opioid-related deaths: a before (2006-10) versus after (2011-13) comparison. Addiction. 2016 May;111(5):883-91. doi: 10.1111/add.13265. PMID: 26642424. Excluded for wrong population.

7. Breedvelt JJF, Tracey DK, Dickenson EC, et al. "Take home" naloxone: what does the evidence base tell us? Drugs Alcohol Today. 2015;15(2):67-75. doi: 10.1108/DAT-03-2015-0017. Excluded; not a study.

8. Buajordet I, Naess AC, Jacobsen D, et al. Adverse events after naloxone treatment of episodes of suspected acute opioid overdose. Eur J Emerg Med. 2004 Feb;11(1):19-23. PMID: 15167188. Excluded for wrong comparator.

9. Cantwell K, Dietze P, Flander L. The relationship between naloxone dose and key patient variables in the treatment of non-fatal heroin overdose in the prehospital setting. Resuscitation. 2005 Jun;65(3):315-9. PMID: 15919568. Excluded for wrong comparator.

10. Centers for Disease Control and Prevention. Increases in Fentanyl Drug Confiscations and Fentanylrelated Overdose Fatalities U.S. Department of Health \& Human Services. Atlanta, GA: 2016. https://emergency.cdc.gov/han/han00 384.asp Accessed December 19, 2016. Excluded; not a study. 
11. Clark AK, Wilder CM, Winstanley EL. A systematic review of community opioid overdose prevention and naloxone distribution programs. J Addict Med. 2014 MayJun;8(3):153-63. doi: 10.1097/ADM.0000000000000034. PMID: 24874759. Excluded for wrong population.

12. Clarke A, Eustace A. External evaluation of the naloxone demonstration project: health service executive. Office HSEHNSI; 2016. Excluded for wrong population.

13. Clarke S, Dargan P. Intravenous bolus or infusion of naloxone in opioid overdose. Emerg Med J. 2002:249-50. PMID: 11971842. Excluded; not a study.

14. Clarke S, Dargan P. Intravenous or intramuscular/subcutaneous naloxone in opioid overdose. Emerg Med J. 2002:249-. PMID: 11971843. Excluded; not a study.

15. Clarke SF, Dargan PI, Jones AL. Naloxone in opioid poisoning: walking the tightrope. Emerg Med J. 2005 Sep;22(9):612-6. PMID: 16113176. Excluded; systematic review or meta-analysis used as a source document to identify individual studies.

16. Creed C. Use of intranasal drug administration in the pre-hospital setting. J Adv Pract Oncol. 2012;4(12):699-704. Excluded; not a study.

17. Dixon P. Managing acute heroin overdose. Emerg Nurse. 2007 May;15(2):30-5. PMID: 17542332. Excluded; not a study.
18. Etherington J, Christenson J, Innes $\mathrm{G}$, et al. Is early discharge safe after naloxone reversal of presumed opioid overdose? CJEM. 2000 Jul;2(3):156-62. PMID: 17621393. Excluded for wrong comparator.

19. Evans A, Snooks H, Russell D, et al. P69 How to stop drug users dying from an overdose: a systematic review of treatment and prevention interventions in the community. J Epidemiol Community Health. 2010;64:A60-A. Excluded; not a study.

20. Faul M, Lurie P, Kinsman J, et al. Multiple naloxone administrations among EMS Providers is increasing. In Press. Excluded for wrong outcome.

21. Fisher R, O'Donnell D, Ray B, et al. Police Officers Can Safely and Effectively Administer Intranasal Naloxone. Prehospital Emergency Care. 2016:1-6. Excluded for wrong outcome.

22. Gjersing L, Bretteville-Jensen AL. Are overdoses treated by ambulance services an opportunity for additional interventions? A prospective cohort study. Addiction. 2015;110(11):1767-74. doi: 10.1111/add.13026. PMID: 2618947. Excluded for wrong population.

23. Glaser A, Arakaki D, Chan GM, et al. Randomised trial of intranasal versus intramuscular naloxone in prehospital treatment for suspected opioid overdose. Med J Aust. 2005 Apr 18;182(8):427; author reply , 9. PMID: 15850442. Excluded; not a study. 
24. Glass PS, Jhaveri RM, Smith LR. Comparison of potency and duration of action of nalmefene and naloxone. Anesth Analg. 1994 Mar;78(3):53641. PMID: 8109774. Excluded for wrong population.

25. Goicoechea C, Sanchez E, Cano C, et al. Analgesic activity and pharmacological characterization of $\mathrm{N}$-[1-phenylpyrazol-3-yl]-N-[1-(2phenethyl)-4-piperidyl] propenamide, a new opioid agonist acting peripherally. Eur J Pharmacol. 2008 Oct 24;595(1-3):22-9. doi: 10.1016/j.ejphar.2008.07.052. PMID: 18706410. Excluded for wrong intervention.

26. Goldfrank L, Weisman RS, Errick $\mathrm{JK}$, et al. A dosing nomogram for continuous infusion intravenous naloxone. Ann Emerg Med. 1986 May;15(5):566-70. PMID: 3963538. Excluded for wrong comparator.

27. Hansen A. Norway tries naloxone in spray form to prevent deaths from drug overdose. BMJ.

2014;348:g1686. doi: 10.1136/bmj.g1686. PMID: 24555965. Excluded; not a study.

28. Heyerdahl F, Hovda KE, Bjornaas MA, et al. Pre-hospital treatment of acute poisonings in Oslo. BMC Emerg. 2008;8:15. doi: 10.1186/1471-227X-8-15. PMID: 19025643. Excluded for wrong intervention.

29. Horowitz Z. Subcutaneous naloxone: a less rude awakening? Acad Emerg Med. 1998 Apr;5(4):283-5. PMID: 9562188. Excluded; not a study.
30. Hussain A, Kimura R, Huang CH. Nasal absorption of naloxone and buprenorphine in rats. Int J Pharm. 1984;21:233-7. Excluded for wrong population.

31. Ishiyama D, Jones J. Towards evidence based emergency medicine: best BETs from the Manchester Royal Infirmary. BET 3: Is nebulised naloxone effective in opioid overdose? Emerg Med J. 2013 Oct;30(10):860. doi: 10.1136/emermed-2013-203100.3. PMID: 24014692. Excluded; not a study.

32. Kaplan JL, Marx JA, Calabro JJ, et al. Double-blind, randomized study of nalmefene and naloxone in emergency department patients with suspected narcotic overdose. Ann Emerg Med. 1999 Jul;34(1):42-50. PMID: 10381993. Excluded for wrong comparator.

33. Kelly AM, Koutsogiannis Z. Intranasal naloxone for life threatening opioid toxicity. Emerg Med J. 2002 Jul;19(4):375. PMID: 12101174. Excluded; not a study.

34. Kerr D, Dietze P, Kelly AM. Intranasal naloxone for the treatment of suspected heroin overdose. Addiction. 2008 Mar;103(3):379-86. doi: 10.1111/j.13600443.2007.02097.x. PMID: 18269360. Excluded; systematic review or meta-analysis used as a source document to identify individual studies. 
35. Kim D, Irwin KS, Khoshnood K. Expanded access to naloxone: options for critical response to the epidemic of opioid overdose mortality. Am J Public Health. 2009 Mar;99(3):402-7. doi: 10.2105/AJPH.2008.136937. PMID: 19150908. Excluded; not a study.

36. Kirane H, Ketteringham M, Bereket $\mathrm{S}$, et al. Awareness and attitudes toward intranasal naloxone rescue for opioid overdose prevention. J Subst Abuse Treat. 2016;69:44-9. PMID: 27568509. Excluded for wrong population.

37. Kitch BB, Portela RC. Effective use of naloxone by law enforcement in response to multiple opioid overdoses. Prehosp Emerg Care. 2016;20(2):226-9. doi: 10.3109/10903127.2015.1076097. PMID: 27002349. Excluded for wrong study design for Key Question.

38. Knowlton A, Weir BW, Hazzard F, et al. EMS runs for suspected opioid overdose: implications for surveillance and prevention. Prehosp Emerg Care. 2013 JulSep;17(3):317-29. doi: 10.3109/10903127.2013.792888. PMID: 23734988. Excluded for wrong outcome.

39. Kouyoumdjian F, Orkin AM, Bingham K, et al. Quality assessment errors and study misclassification threaten systematic eview validity: community opioid overdose prevention and naloxone distribution programs review. J Addict Med. 2015;9(6):502-3. doi: 10.1097/ADM.0000000000000161. PMID: 26517325. Excluded; not a study.
40. Lindstrom HA, Clemency BM, Snyder R, et al. Prehospital naloxone administration as a public health surveillance tool: a retrospective validation study. Prehosp Disaster Med. 2015 Aug;30(4):385-9. doi: 10.1017/S1049023X15004793. PMID: 26061280. Excluded for wrong outcome.

41. Lobmaier PP, Clausen T. Radical red tape reduction by government supported nasal naloxone: the Norwegian pilot project is innovative, safe and an important contribution to further development and dissemination of take-home naloxone. Addiction. 2016 Apr;111(4):586-7. doi: 10.1111/add.13261. PMID: 26995169. Excluded; not a study.

42. Loimer N, Hofmann P, Chaudhry HR. Nasal administration of naloxone is as effective as the intravenous route in opiate addicts. Int J Addict. 1994 Apr;29(6):819-27. PMID: 8034388. Excluded for wrong population.

43. M J, C S. Notes on naloxone...August issue article "Armed with Naloxone: The first responders' ultimate weapon in the war on opioid use? JEMS.

2014;39(11):12-. Excluded; not a study.

44. Massachusetts Department of Public Health. Opioid-related EMS Transports Massachusetts Residents: 2013-2015. May, 2016. http://www.mass.gov/eohhs/docs/dp h/quality/drugcontrol/county-levelpmp/emergency-medical-servicesdata-may-2016.pdf Accessed Decmeber 19, 2016. Excluded for wrong outcome. 
45. McDermott C, Collins NC. Prehospital medication administration: a randomised study comparing intranasal and intravenous routes. Emerg Med Int. 2012;2012:476161. doi: 10.1155/2012/476161. PMID: 22953064. Excluded for wrong population.

46. McDonald R, Strang J. Are takehome naloxone programmes effective? Systematic review utilizing application of the Bradford Hill criteria. Addiction. 2016 Jul;111(7):1177-87. doi: 10.1111/add.13326. PMID: 27028542. Excluded for wrong population.

47. Merlin MA, Ariyaprakai N, Arshad FH. Assessment of the safety and ease of use of the naloxone autoinjector for the reversal of opioid overdose. Open Access Emerg Med. 2015;7:21-4. doi: 10.2147/OAEM.S82133. PMID: 27147886. Excluded; not a study.

48. Naloxone distribution saves more than 400 lives in SF OD project. ADAW. 2010;22(42):3-4. Excluded; not a study.

49. Naloxone distribution saves more than 400 lives in SF overdose project. Brown Univ Dig Addict Theory Appl. 2010;29(12):4-5. Excluded; not a study.

50. Naloxone for heroin overdose reversal. Ann Intern Med. 2013;158(1):I-30. doi: 10.7326/0003-4819-158-1201301010-00001. Excluded for wrong population.
51. News: clinical digest: a weekly round-up of the latest research and scientific reports from the nursing and medical journals. Intranasal naloxone effective in patients who overdose. Nurs Stand. 2005;19(24):10-. Excluded; not a study.

52. Neale J, Strang J. Naloxone--does over-antagonism matter? Evidence of iatrogenic harm after emergency treatment of heroin/opioid overdose. Addiction. 2015 Oct;110(10):164452. doi: 10.1111/add.13027. PMID: 26119038. Excluded for wrong population.

53. Nielsen S, Menon N, Larney S, et al. Community pharmacist knowledge, attitudes and confidence regarding naloxone for overdose reversal. Addiction. 2016 PMID: 27367125. Excluded for wrong population.

54. Osterwalder JJ. Patients intoxicated with heroin or heroin mixtures: how long should they be monitored? Eur J Emerg Med. 1995 Jun;2(2):97-101. PMID: 9422191. Excluded for wrong intervention.

55. Osterwalder JJ. Naloxone--for intoxications with intravenous heroin and heroin mixtures--harmless or hazardous? a prospective clinical study. J Toxicol Clin Toxicol. 1996;34(4):409-16. PMID: 8699555. Excluded for wrong comparator.

56. Purviance D, Ray B, Tracy A, et al. Law enforcement attitudes towards naloxone following opioid overdose training. Subst Abus. 2016 PMID: 27715714. Excluded for wrong population. 
57. Roberts JR. Providing naloxone to non-medical personnel can prevent OD deaths without increasing abuse. Emergency Medicine News. 2014;36(9):12-4. Excluded; not a study.

58. Robertson TM. Intranasal versus intravenous naloxone for prehospital narcotic overdose. Academic Emergency Medicine. 2005;12:1667. Excluded; not a study.

59. Romac DR. Safety of prolonged, high-dose infusion of naloxone hydrochloride for severe methadone overdose. Clin Pharm. 1986 Mar;5(3):251-4. PMID: 3956131. Excluded for wrong study design for Key Question.

60. Rowe C, Santos GM, Vittinghoff E, et al. Predictors of participant engagement and naloxone utilization in a community-based naloxone distribution program. Addiction. 2015 Aug;110(8):1301-10. doi: 10.1111/add.12961. PMID: 25917125. Excluded for wrong population.

61. Rupreht J, Dworacek B, Oosthoek H, et al. Physostigmine versus naloxone in heroin-overdose. J Toxicol Clin Toxicol. 1983;21(3):387-97. PMID: 6676478. Excluded for wrong comparator.

62. Salvucci A, Jr. IN vs. IM naloxone. Emergency Medical Services. 2005;34(8):64-6. Excluded; not a study.
63. Sanaei-Zadeh H. Is transdermal iontophoretic delivery of naloxone sufficient for the management of intoxication in opioid-overdosed patients? Int J Pharm. 2012 May 30;428(1-2):187. doi: 10.1016/j.ijpharm.2012.02.045. PMID: 22426321. Excluded; not a study.

64. Smith DA, Leake L, Loflin JR, et al. Is admission after intravenous heroin overdose necessary? Ann Emerg Med. 1992 Nov;21(11):1326-30. PMID: 1416327. Excluded for wrong population.

65. Sutter ME, Gerona RR, Davis MT, et al. Fatal Fentanyl: One Pill Can Kill. Acad Emerg Med. 2016 Jun 20doi: 10.1111/acem.13034. PMID: 27322591. Excluded for wrong outcome.

66. Walley AY, Xuan Z, Hackman HH, et al. Opioid overdose rates and implementation of overdose education and nasal naloxone distribution in Massachusetts: interrupted time series analysis. BMJ. 2013;346:f174. doi: 10.1136/bmj.f174. PMID: 23372174. Excluded for wrong population.

67. Wasiak J, Clavisi O. Is subcutaneous or intramuscular naloxone as effective as intravenous naloxone in the treatment of life-threatening heroin overdose? Med J Aust. 2002 May 20;176(10):495. PMID: 12065016. Excluded; not a study.

68. Willman MW, Liss DB, Schwarz ES, et al. Do heroin overdose patients require observation after receiving naloxone? Clin Toxicol. 2017 Feb;55(2):81-7. PMID: 27849133. Excluded; wrong population. 
69. Yealy DM, Paris PM, Kaplan RM, et al. The safety of prehospital naloxone administration by paramedics. Ann Emerg Med. 1990
Aug;19(8):902-5. PMID: 2372173.

Excluded for wrong comparator. 


\section{Appendix D. Data Abstraction}

Table D-1. Key Question 1: Data abstraction of randomized controlled trials

\begin{tabular}{|c|c|c|c|c|c|}
\hline Author, Year & $\begin{array}{l}\text { Key } \\
\text { Question }\end{array}$ & $\begin{array}{l}\text { Country } \\
\text { Setting Study } \\
\text { Years }\end{array}$ & Eligibility Criteria & $\begin{array}{l}\text { Intervention Characteristics (experimental vs. } \\
\text { control groups: dose, duration and route of } \\
\text { treatment; training/background of personnel } \\
\text { administering drug; timing of administration) }\end{array}$ & $\begin{array}{l}\text { Number of Treatment and } \\
\text { Control Subjects } \\
\text { (randomized, analyzed, lost to } \\
\text { followup) }\end{array}$ \\
\hline Kerr, 2009 & KQ1 & $\begin{array}{l}\text { Australia } \\
\text { Prehospital } \\
\text { ambulance } \\
\text { service } \\
2006-2008\end{array}$ & $\begin{array}{l}\text { Patients requiring treatment for } \\
\text { suspected opioid overdose: } \\
\text { Altered conscious state } \\
\text { (pinpoint pupils, respirations } \\
<10 / \text { minute) } \\
\text { GCS } \leq 12 \\
\text { No major facial trauma, } \\
\text { blocked nasal passages, or } \\
\text { epistaxis }\end{array}$ & $\begin{array}{l}\text { A: Naloxone intranasal } 2 \mathrm{mg} / 1 \mathrm{ml} \text { with mucosal } \\
\text { atomization device, } 1 \mathrm{mg} \text { each nostril }(\mathrm{n}=83) \\
\text { B: Naloxone intramuscular } 2 \mathrm{mg} / 5 \mathrm{ml} \text { 'min-i-jet' } \\
\text { preparation } x 1(\mathrm{n}=89) \\
\text { Paramedics administered naloxone. } \\
\text { Both groups received naloxone intramuscular } 0.8 \\
\text { mg for failure to respond after } 10 \text { minutes }\end{array}$ & $\begin{array}{l}\text { Randomized: } 178 \text { (88 vs. 90) } \\
\text { Analyzed: } 169 \text { (83 vs. } 89 \text { ) } \\
\text { Lost to followup: None reported }\end{array}$ \\
\hline Kelly, 2005 & KQ1 & $\begin{array}{l}\text { Australia } \\
\text { Ambulance } \\
\text { service } \\
2002-2003\end{array}$ & $\begin{array}{l}\text { Suspected opiate overdose: } \\
\leq 10 \text { respirations per minute } \\
\text { Not rousable }\end{array}$ & $\begin{array}{l}\text { A: Naloxone intranasal } 2 \mathrm{mg} / 5 \mathrm{~mL} \text { with mucosal } \\
\text { atomization device, } 1 \mathrm{mg} \text { each nostril }(\mathrm{n}=84) \\
\text { B: Naloxone intramuscular } 2 \mathrm{mg} \times 1 \text { (preparation } \\
\text { not described) }(\mathrm{n}=71) \\
\text { Paramedics administered naloxone. } \\
\text { Both groups received naloxone intramuscular } 0.8 \\
\text { mg for failure to respond after } 8 \text { minutes }\end{array}$ & $\begin{array}{l}\text { Randomized: } 182 \text { (98 vs. } 84) \\
\text { Analyzed: } 155 \text { (84 vs. } 71) \\
\text { Lost to followup: None reported; } 4 \\
\text { vs. } 8 \text { with incomplete data. }\end{array}$ \\
\hline $\begin{array}{l}\text { Sabzghabaee, } \\
2014\end{array}$ & KQ1 & $\begin{array}{l}\text { Iran } \\
\text { Emergency } \\
\text { Department } \\
2007\end{array}$ & $\begin{array}{l}\text { Age 15-50 years } \\
\text { Suspected opioid overdose: } \\
\text { Myotic pupils } \\
\text { Loss of consciousness with or } \\
\text { without respiratory depression }\end{array}$ & $\begin{array}{l}\text { A: Naloxone intranasal } 0.4 \mathrm{mg} \text { in } 2 \mathrm{~mL} \\
\text { B: Naloxone intravenous } 0.4 \mathrm{mg} \\
\text { All patients who failed to respond within } 5 \\
\text { minutes were given a further } 0.4 \mathrm{mg} \text { naloxone by } \\
\text { the same administration route as the first dose }\end{array}$ & $\begin{array}{l}\text { Randomized: } 100 \text { (50 vs. 50) } \\
\text { Analyzed: Not reported } \\
\text { Lost to followup: Not reported }\end{array}$ \\
\hline
\end{tabular}




\begin{tabular}{|c|c|c|c|}
\hline Author, Year & $\begin{array}{l}\text { Population Characteristics } \\
\text { (Age, sex, race, psychiatric } \\
\text { comorbidities, prior overdose } \\
\text { episodes) }\end{array}$ & $\begin{array}{l}\text { Exposure Characteristics (type of opioid } \\
\text { involve, dose of opioid involved, other } \\
\text { contributing drugs, time since overdose) }\end{array}$ & Results \\
\hline Kerr, 2009 & $\begin{array}{l}\text { Age: } 31 \text { vs. } 32 \text { years } \\
\text { Male: } 77 \% \text { vs. } 71 \% \text { Race: Not } \\
\text { reported } \\
\text { Psychiatric comorbidities: Not } \\
\text { reported } \\
\text { Prior overdose episodes: Not } \\
\text { reported }\end{array}$ & $\begin{array}{l}\text { Type of opioid: Not reported } \\
\text { Dose of opioid: Not reported Concomitant } \\
\text { alcohol: } 30 \% \text { vs. } 35 \% \text { Concomitant drugs: } 22 \% \\
\text { vs. } 9.0 \% \text { ( } p<0.05 \text { ) Concomitant alcohol } \pm \text { drugs: } \\
47 \% \text { vs. } 37 \% \text { Time since overdose: Not reported }\end{array}$ & 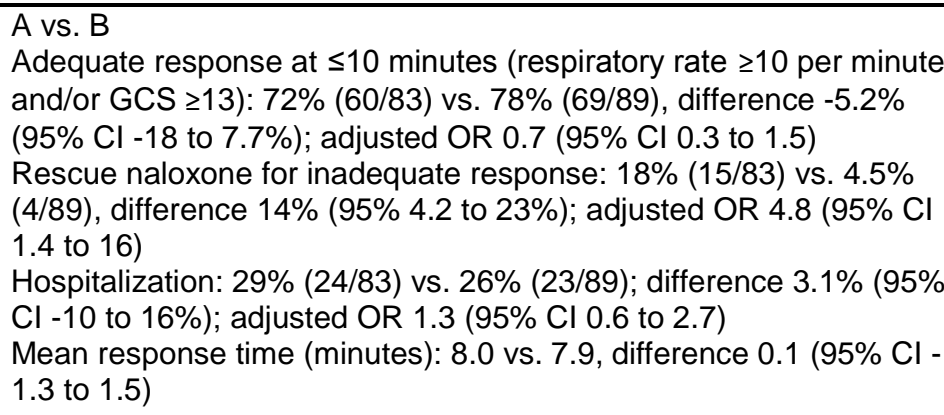 \\
\hline Kelly, 2005 & $\begin{array}{l}\text { Age: } 28 \text { vs. } 30 \text { years } \\
\text { Male: } 70 \% \text { vs. } 73 \% \text { Race: Not } \\
\text { reported } \\
\text { Psychiatric comorbidities: Not } \\
\text { reported } \\
\text { Prior overdose episodes: Not } \\
\text { reported }\end{array}$ & $\begin{array}{l}\text { Type of opioid: Not reported } \\
\text { Dose of opioid: Not reported } \\
\text { Suspicion of concomitant drugs/alcohol: } 37 \% \text { vs. } \\
28 \% \\
\text { Time since overdose: Not reported }\end{array}$ & $\begin{array}{l}\text { A vs. B } \\
\text { Spontaneous respirations within } 8 \text { minutes: } 63 \% \text { (53/84) vs. } 82 \% \\
\text { ( } 58 / 71) \text {, OR } 0.38 \text { ( } 95 \% \mathrm{Cl} 0.18 \text { to } 0.81) \\
\text { Patients requiring rescue naloxone after } 8 \text { minutes: } 26 \% \text { (22/84) vs. } \\
13 \%(9 / 71), \text { OR } 2.4(95 \% \mathrm{Cl} 1.0 \text { to } 5.7) \\
\text { Glasgow Coma Score }>11 \text { at } 8 \text { minutes: } 57 \%(48 / 84) \text { vs. } 72 \% \\
\text { ( } 51 / 71), \text { OR } 0.52(95 \% \mathrm{Cl} 0.27 \text { to } 1.0) \\
\text { Transported to hospital: } 17 \%(14 / 84) \text { vs. } 21 \% \text { (15/71), OR } 0.75 \text {, } \\
95 \% \text { Cl } 0.33 \text { to } 1.68 \\
\text { Time to respirations }>10 / \text { minute (minutes): } 8 \text { vs. } 6 \text { minutes, } p=0.006 \\
\text { Time to GCS score }>11: p=0.38 \text {, data not reported }\end{array}$ \\
\hline $\begin{array}{l}\text { Sabzghabaee, } \\
2014\end{array}$ & $\begin{array}{l}\text { Age: } 30 \text { vs. } 33 \text { years } \\
\text { Male: } 78 \% \text { vs. } 74 \% \text { Race: Not } \\
\text { reported } \\
\text { Psychiatric comorbidities: Not } \\
\text { reported } \\
\text { Prior overdose episodes: Not } \\
\text { reported }\end{array}$ & $\begin{array}{l}\text { Type of opioid: Diphenoxylate } 0 \text { vs. } 8 \% \\
\text { Crack (in Iran Crack contains heroin with other } \\
\text { opiates): } 4 \% \text { vs. } 0 \\
\text { Methadone: } 16 \% \text { vs. } 20 \% \text { Heroin: } 28 \% \text { vs. } 24 \% \\
\text { Opium: } 44 \% \text { vs. } 48 \% \\
\text { Dose involved: Not reported Concomitant drugs: } \\
\text { Not reported Time since overdose: Not reported }\end{array}$ & $\begin{array}{l}\text { A vs. B: } \\
\text { Systolic blood pressure: } 106 \pm 14.7 \text { vs. } 112 \pm 9.6, p=0.18 \\
\text { Diastolic blood pressure: } 78 \pm 7.1 \text { vs. } 77 \pm 4.5, p=0.18 \\
\text { Heart rate (per minute): } 90 \pm 8.3 \text { vs. } 97 \pm 12.9, p=0.003 \\
\text { Respirator rate (per minute): } 18 \pm 2.4 \text { vs. } 19 \pm 2.8, p=0.17 \\
\text { Glasgow Coma Scale: } 14.3 \pm 0.73 \text { vs. } 13.2 \pm 1.5, p<0.001 \\
\text { Length of hospital stay (days): } 1.53 \pm 0.16 \text { vs. } 1.2 \pm 0.15, p=0.15\end{array}$ \\
\hline
\end{tabular}




\begin{tabular}{|c|c|c|c|c|}
\hline Author, Year & $\begin{array}{l}\text { Duration of } \\
\text { Followup }\end{array}$ & Adverse Events & Funding Source & Comments \\
\hline Kerr, 2009 & Out-of-hospital & 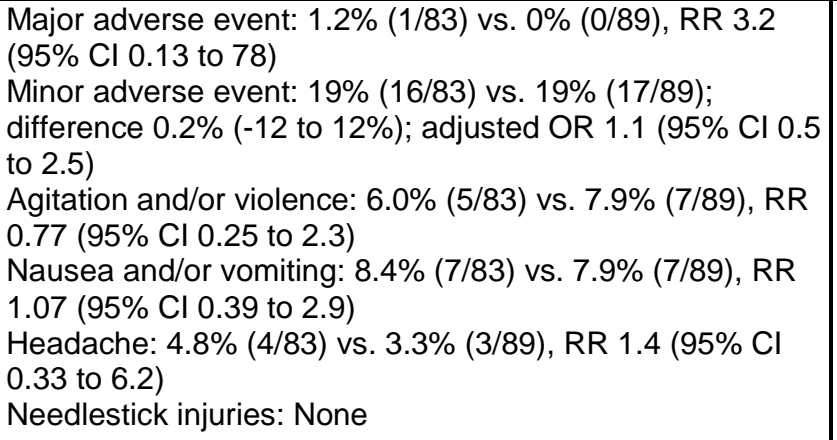 & $\begin{array}{l}\text { Drug Policy and } \\
\text { Services, Department } \\
\text { of Human Services, } \\
\text { Melbourne, Victoria, } \\
\text { Australia }\end{array}$ & $\begin{array}{l}\text { Adjusted risk estimates adjusted for age, } \\
\text { gender, and suspected concomitant alcohol } \\
\text { and/or drugs; results similar to unadjusted } \\
\text { Site of IM administration not specified, } \\
\text { difference between the IN and IM arms with } \\
\text { regard to rescue naloxone despite no } \\
\text { response at } 10 \text { minutes, possible bias } \\
\text { favoring more use of rescue in IN arm. } \\
\text { Unblinded study. No concerns about } \\
\text { randomization or concealment. }\end{array}$ \\
\hline Kelly, 2005 & Out-of-hospital & $\begin{array}{l}\text { Major adverse event: } 0 \%(0 / 84) \text { vs. } 0 \%(0 / 71) \\
\text { Minor adverse event: } 12 \%(10 / 84) \text { vs. } 21 \%(15 / 71), \mathrm{RR} \\
0.56 \text { ( } 95 \% \mathrm{Cl} 0.27 \text { to } 1.18) \\
\text { Agitation and/or irritation: } 2.4 \%(2 / 84) \text { vs. } 14 \%(10 / 71) \text {; RR } \\
0.19(95 \% \mathrm{Cl} 0.04 \text { to } 0.83) \\
\text { Nausea and/or vomiting: } 7.1 \%(6 / 84) \text { vs. } 5.6 \%(4 / 71), \mathrm{RR} \\
1.3(95 \% \mathrm{Cl} 0.37 \text { to } 4.3) \\
\text { Headache: } 0 \%(0 / 84) \text { vs. } 2.8 \%(2 / 71) \text {, RR } 0.17(95 \% \mathrm{Cl} \\
0.008 \text { to } 3.5) \\
\text { Tremor: } 1.2 \%(1 / 84) \text { vs. } 1.4 \%(1 / 71), \mathrm{RR} 0.85(95 \% \mathrm{Cl} 0.05 \\
\text { to } 13) \\
\text { Sweating: } 1.2 \%(1 / 84) \text { vs. } 0 \%(0 / 71), \mathrm{RR} 2.5(95 \% \mathrm{Cl} 0.11 \\
\text { to } 61)\end{array}$ & $\begin{array}{l}\text { William Buckland } \\
\text { Foundation }\end{array}$ & $\begin{array}{l}\text { Site of IM administration not specified, } \\
\text { difference between the IN and IM arms with } \\
\text { regard to rescue naloxone despite no } \\
\text { response at } 8 \text { minutes, possible bias } \\
\text { favoring more use of rescue in IN arm. } \\
\text { Unblinded study. No concerns about } \\
\text { randomization or concealment. Same } \\
\text { formulation used in both groups } 5 \mathrm{mg} / 2 \mathrm{ml} \text {. } \\
\text { Sample size predetermined to be } 166 \text { for } 60 \\
\text { second difference but mITT analysis had } \\
155 \text { cases. GCS and need for rescue nearly } \\
\text { achieved statistical significance. }\end{array}$ \\
\hline $\begin{array}{l}\text { Sabzghabaee, } \\
2014\end{array}$ & $\begin{array}{l}\text { Upon hospital } \\
\text { discharge }\end{array}$ & $\begin{array}{l}\text { A vs. B } \\
\text { Agitation: } 0 \text { vs. } 12\end{array}$ & University support & $\begin{array}{l}\text { The study does not report number of } \\
\text { excluded patients, but states that patients } \\
\text { failing to respond to the first } 0.4 \text { mg } \\
\text { naloxone with an increased level of } \\
\text { consciousness or a reversal of respiratory } \\
\text { depression were excluded from the study. }\end{array}$ \\
\hline
\end{tabular}


Table D-2. Key Question 1: Data abstraction of observational studies

\begin{tabular}{|c|c|c|c|c|c|}
\hline $\begin{array}{l}\text { Author, Year } \\
\text { Study Design }\end{array}$ & $\begin{array}{l}\text { Key } \\
\text { Question }\end{array}$ & $\begin{array}{l}\text { Country } \\
\text { Setting Study } \\
\text { Years }\end{array}$ & Eligibility Criteria & $\begin{array}{l}\text { Intervention Characteristics by Comparison } \\
\text { Group (dose, duration and route of } \\
\text { treatment; training/background of personnel } \\
\text { administering drug; timing of } \\
\text { administration) }\end{array}$ & $\begin{array}{l}\text { Screened } \\
\text { Eligible } \\
\text { Enrolled } \\
\text { Analyzed } \\
\text { Loss to Followup } \\
\end{array}$ \\
\hline $\begin{array}{l}\text { Merlin, } 2010 \\
\text { Retrospective cohort }\end{array}$ & KQ1 & $\begin{array}{l}\text { United States } \\
\text { Out-of-hospital } \\
\text { 2005-2007 }\end{array}$ & $\begin{array}{l}\text { Opioid intoxication determined } \\
\text { by } \\
\text { at least one of the following: } \\
\text { Patient admission of illegal or } \\
\text { nontherapeutic opioid use; } \\
\text { Witness testimony; } \\
\text { Evidence of opioid use observed } \\
\text { by paramedics; } \\
\text { Positive urine toxicologic screen } \\
\text { for opioids } \\
\text { Excluded patients with cardiac } \\
\text { arrest, intubation before } \\
\text { naloxone administration, } \\
\text { sedation by paramedics before } \\
\text { naloxone administration }\end{array}$ & $\begin{array}{l}\text { A: Intravenous naloxone between } 0.4 \text { and } 2.0 \\
\text { mg ( } n=55) \\
\text { B: Intranasal naloxone at } 1 \mathrm{mg} \text { per nostril at the } \\
\text { discretion of the paramedics ( } \mathrm{n}=38 \text { ) } \\
\text { Naloxone administered by Advanced Life } \\
\text { Support personnel }\end{array}$ & $\begin{array}{l}\text { Screened: } 344 \\
\text { Eligible: } 96 \\
\text { Enrolled: } 96 \\
\text { Analyzed: } 93 \text { (55 vs. } 38 \text { ) } \\
\text { Lost to followup: } 0\end{array}$ \\
\hline $\begin{array}{l}\text { Robertson, } 2009 \\
\text { Retrospective cohort }\end{array}$ & KQ1 & $\begin{array}{l}\text { United States } \\
\text { Out-of-hospital } \\
\text { 2003-2004 }\end{array}$ & $\begin{array}{l}\text { Patients treated with naloxone } \\
\text { for } \\
\text { suspected opioid overdose: } \\
\text { clinically suspected of opiate } \\
\text { intoxication and } \leq 8 \text { breaths per } \\
\text { minute }\end{array}$ & $\begin{array}{l}\text { A: Intravenous naloxone as first line treatment, } \\
\text { dose protocol not reported ( } \mathrm{n}=104) \\
\text { B: Intranasal naloxone as first line treatment, } 2 \\
\mathrm{mg}(1 \mathrm{mg} \text { per nostril) using mucosal atomizer } \\
\text { device }(\mathrm{n}=50) \\
\text { EMS administered naloxone, training not } \\
\text { specified } \\
\text { Patients in first year were treated with } \\
\text { intravenous naloxone and patients in last } 5 \\
\text { months were treated with intranasal naloxone. }\end{array}$ & $\begin{array}{l}\text { Screened: Not reported } \\
\text { Eligible: } 154 \\
\text { Enrolled: } 154 \\
\text { Analyzed: } 154 \text { (104 vs. 50) } \\
\text { Lost to followup: } 0\end{array}$ \\
\hline
\end{tabular}




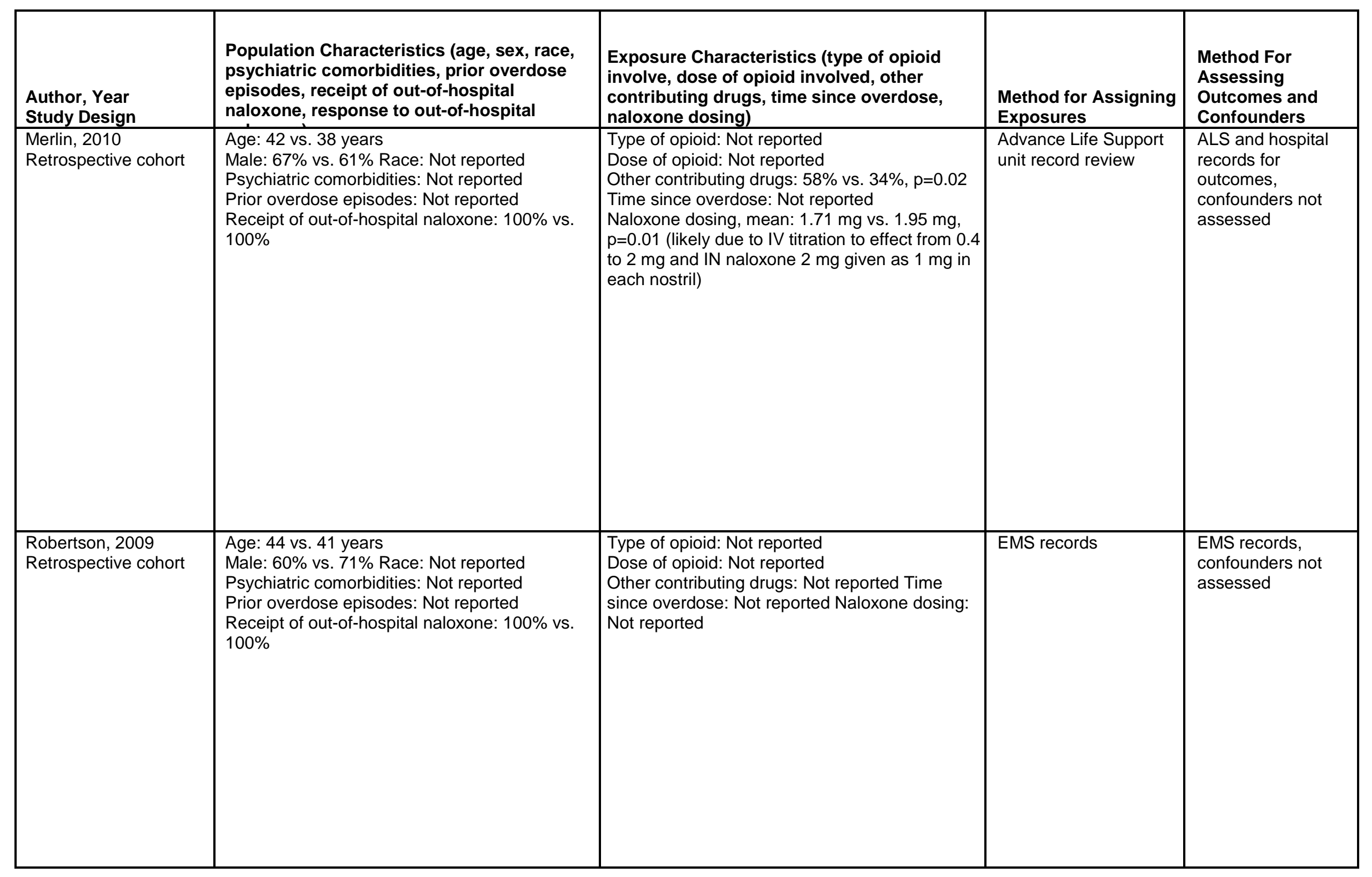




\begin{tabular}{|c|c|c|c|c|c|}
\hline $\begin{array}{l}\text { Author, Year } \\
\text { Study Design }\end{array}$ & $\begin{array}{l}\text { Duration of } \\
\text { Followup }\end{array}$ & $\begin{array}{l}\text { Adjusted Variables For } \\
\text { Statistical Analysis }\end{array}$ & Results & $\begin{array}{l}\text { Funding } \\
\text { Source }\end{array}$ & Comments \\
\hline $\begin{array}{l}\text { Merlin, } 2010 \\
\text { Retrospective cohort }\end{array}$ & Hospital discharge & No adjustment performed & $\begin{array}{l}\text { A vs. B: } \\
\text { Median change in breaths per minute } \\
\text { from baseline: } 6 \text { vs. } 4 \text {, difference } 2(95 \% \\
\mathrm{Cl} \text { - } \\
0.001 \text { to } 5), p=0.08 \\
\text { Median change in Glasgow Coma Scale } \\
\text { from baseline: } 4 \text { vs. } 3 \text {, difference } 1(95 \% \\
\mathrm{Cl}-0.001 \text { to } 3), p=0.19\end{array}$ & $\begin{array}{l}\text { No grants or } \\
\text { financial } \\
\text { support }\end{array}$ & $\begin{array}{l}\text { Only ALS personnel may } \\
\text { administer naloxone in this site. } \\
\text { Median dose in the both groups } \\
\text { was } 2 \text { mg. Mean was slightly } \\
\text { different as noted. Formulation } \\
\text { not specified. Complicated } \\
\text { analysis suspect related to } \\
\text { retrospective study design. } \\
\text { Study done in New Jersey, NJ, } \\
\text { USA. }\end{array}$ \\
\hline $\begin{array}{l}\text { Robertson, } 2009 \\
\text { Retrospective cohort }\end{array}$ & End of EMS contact & No adjustment performed & $\begin{array}{l}\text { A vs. B: } \\
\text { Positive clinical response (increase in } \\
\text { respirations of at least } 6 \text { breaths/minute or } \\
\text { in GCS of at least } 6 \text { points): } 58 / 104 \\
(56 \%) \text { vs. } 33 / 50(66 \%), p=0.3) \\
\text { Time from naloxone administration to } \\
\text { effect: } 8.1 \text { vs. } 12.9 \text { minutes, } p=0.02 \\
\text { Time from patient contact to effect: } 20.7 \\
\text { vs. } 20.3 \text { minutes, } p=0.9 \\
\text { Second dose of naloxone given: } 19 / 104 \\
(18 \%) \text { vs. } 17 / 50(34 \%), p=0.05\end{array}$ & Not reported & $\begin{array}{l}\text { EMS records in this system were } \\
\text { all electronic. Definition of clinical } \\
\text { response increase in RR of } 6 \text { or } \\
\text { GCS of } 6 \text {. There were } 3 \text { IN } \\
\text { patients that received rescue } \\
\text { naloxone via an alternate route. } \\
\text { Although retrospective, looked at } \\
\text { a protocol change from IV as first } \\
\text { line to IN as first line. Similar in a } \\
\text { way to Wanger which was } \\
\text { prospective. Study done in } \\
\text { Fresno, CA, USA. }\end{array}$ \\
\hline
\end{tabular}




\begin{tabular}{|c|c|c|c|c|c|}
\hline $\begin{array}{l}\text { Author, Year } \\
\text { Study Design }\end{array}$ & $\begin{array}{l}\text { Key } \\
\text { Question }\end{array}$ & $\begin{array}{l}\text { Country } \\
\text { Setting Study } \\
\text { Years }\end{array}$ & Eligibility Criteria & $\begin{array}{l}\text { Intervention Characteristics by Comparison } \\
\text { Group (dose, duration and route of } \\
\text { treatment; training/background of } \\
\text { personnel } \\
\text { administering drug; timing of } \\
\text { administration) }\end{array}$ & $\begin{array}{l}\text { Screened } \\
\text { Eligible } \\
\text { Enrolled } \\
\text { Analyzed } \\
\text { Loss to Followup } \\
\end{array}$ \\
\hline $\begin{array}{l}\text { Sporer, } 1996 \\
\text { Retrospective cohort }\end{array}$ & KQ1 & $\begin{array}{l}\text { United States } \\
\text { Out- of- } \\
\text { hospital } \\
1993\end{array}$ & $\begin{array}{l}\text { Presumed opioid overdose } \\
\text { required at least } 3 \text { of the } \\
\text { following: } \\
\text { Circumstantial evidence of } \\
\text { parenteral drug use Respiratory } \\
\text { rate }<6 \text { per minute Cyanosis } \\
\text { prior to oxygenation } \\
\text { Glasgow Coma Scale score } \leq 12 \\
\text { Pinpoint pupils }\end{array}$ & $\begin{array}{l}\text { A: Intravenous naloxone } 2 \mathrm{mg} \\
\text { B: Intramuscular naloxone } 2 \mathrm{mg} \\
\text { Repeat dose given if no response is seen in 1-2 } \\
\text { minutes }\end{array}$ & $\begin{array}{l}\text { Screened: } 1,856 \\
\text { Eligible: } 609 \\
\text { Analyzed: } 609 \\
\text { Lost to followup: Not } \\
\text { reported, but } 166 \text { patients } \\
\text { not transported to the } \\
\text { hospital do not have } \\
\text { clinical outcomes }\end{array}$ \\
\hline $\begin{array}{l}\text { Wanger, } 1998 \\
\text { Prospective cohort with } \\
\text { historical control }\end{array}$ & KQ1 & $\begin{array}{l}\text { Canada } \\
\text { Out- of- } \\
\text { hospital IV } \\
\text { naloxone: June } \\
\text { 1-30, 1996 } \\
\text { SQ naloxone: } \\
\text { July 1- } \\
\text { September 1, } \\
1996\end{array}$ & $\begin{array}{l}\text { Out-of-hospital patients with } \\
\text { presumed opioid overdose who } \\
\text { received naloxone: } \\
\text { Decreased consciousness } \\
\text { History suggestive of opioid use } \\
\text { Respiratory rate }<10 \text { breaths per } \\
\text { minute } \\
\text { Excluded patients in cardiac } \\
\text { arrest }\end{array}$ & $\begin{array}{l}\text { A: Subcutaneous naloxone } 0.8 \mathrm{mg} \text { into upper } \\
\text { arm or thigh, with } 0.4 \mathrm{mg} \text { IV available if no } \\
\text { improvement after } 5 \text { minutes ( } \mathrm{n}=139) \\
\text { B: Intravenous naloxone } 0.4 \mathrm{mg} \text {, with an } \\
\text { additional } 0.4 \mathrm{mg} \text { available if no improvement } \\
\text { after } 5 \text { minutes ( } \mathrm{n}=83 \text { ) } \\
\text { Naloxone administered by British Columbia } \\
\text { Ambulance Service attendants (basic life } \\
\text { support, IV and advanced life support levels) }\end{array}$ & $\begin{array}{l}\text { Screened: Not reported } \\
\text { Eligible: Not reported } \\
\text { Enrolled: } 222 \\
\text { Analyzed: } 196 \text { (122 vs. } 74 \text { ) } \\
\text { Lost to followup: Not } \\
\text { reported }\end{array}$ \\
\hline
\end{tabular}




\begin{tabular}{|c|c|c|c|c|}
\hline $\begin{array}{l}\text { Author, Year } \\
\text { Study Design }\end{array}$ & $\begin{array}{l}\text { Population Characteristics (age, sex, race, } \\
\text { psychiatric comorbidities, prior overdose } \\
\text { episodes, receipt of out-of-hospital } \\
\text { naloxone, response to out-of-hospital }\end{array}$ & $\begin{array}{l}\text { Exposure Characteristics (type of opioid } \\
\text { involve, dose of opioid involved, other } \\
\text { contributing drugs, time since overdose, } \\
\text { naloxone dosing) }\end{array}$ & $\begin{array}{l}\text { Method for Assigning } \\
\text { Exposures }\end{array}$ & $\begin{array}{l}\text { Method For } \\
\text { Assessing } \\
\text { Outcomes and } \\
\text { Confounders } \\
\end{array}$ \\
\hline $\begin{array}{l}\text { Sporer, } 1996 \\
\text { Retrospective cohort }\end{array}$ & $\begin{array}{l}\text { Not reported by treatment group } \\
\text { Age: <20: 3\%; 20-29: 15\%; 30-39: 40\%; 40-49: } \\
\text { 34\%; >50: } \\
9 \% \text { Male: } \\
\text { 78\% } \\
\text { Race: Unknown: 51\%; White: 28\%; Black: } \\
\text { 14\%; Hispanic: } 6 \% ; \text { Asian: } 1 \% \\
\text { Psychiatric comorbidities: Not reported } \\
\text { Prior overdose episodes: Not reported } \\
\text { Receipt of out-of-hospital naloxone: } \\
99.8 \%\end{array}$ & $\begin{array}{l}\text { Type of opioid: IV opioid: } 58 \% \text {; Unknown: } 40 \% \\
\text { Dose of opioid: Not reported } \\
\text { Other contributing drugs: Not reported } \\
\text { Time since overdose: Not reported } \\
\text { Naloxone dosing: } 0.4 \mathrm{mg}: 1 \% ; 1 \mathrm{mg}: 6 \% ; 2 \mathrm{mg} \text { : } \\
\text { 58\%; } 3-4 \mathrm{mg}: 28 \% \text {; }>4 \mathrm{mg}: 7 \%\end{array}$ & $\begin{array}{l}\text { EMS, hospital and } \\
\text { medical } \\
\text { examiner } \\
\text { records }\end{array}$ & $\begin{array}{l}\text { EMS records, } \\
\text { confounders not } \\
\text { assessed }\end{array}$ \\
\hline $\begin{array}{l}\text { Wanger, } 1998 \\
\text { Prospective cohort } \\
\text { with historical control }\end{array}$ & $\begin{array}{l}\text { A vs. B } \\
\text { Age: } 33 \text { vs. } 36 \\
\text { years Male: } 80 \% \\
\text { vs. } 78 \% \text { Race: Not } \\
\text { reported } \\
\text { Psychiatric comorbidities: Not reported } \\
\text { Prior overdose episodes: Not reported } \\
\text { Receipt of out-of-hospital naloxone: } \\
100 \%\end{array}$ & $\begin{array}{l}\text { Type of opioid: Not reported } \\
\text { Dose of opioid: Not reported } \\
\text { Other contributing drugs: Not reported } \\
\text { Time since overdose: Not reported } \\
\text { Naloxone dosing: Patients requiring at least } 2 \\
\text { doses of naloxone prior to hospital arrival: } 15 \% \\
\text { vs. } 35 \%\end{array}$ & $\begin{array}{l}\text { Ambulance service } \\
\text { records }\end{array}$ & $\begin{array}{l}\text { Ambulance service } \\
\text { records, } \\
\text { confounders not } \\
\text { assessed }\end{array}$ \\
\hline
\end{tabular}




\begin{tabular}{|c|c|c|c|c|c|}
\hline $\begin{array}{l}\text { Author, Year } \\
\text { Study Design }\end{array}$ & $\begin{array}{l}\text { Duration of } \\
\text { Followup }\end{array}$ & $\begin{array}{l}\text { Adjusted Variables For } \\
\text { Statistical Analysis }\end{array}$ & Results & $\begin{array}{l}\text { Funding } \\
\text { Source }\end{array}$ & Comments \\
\hline $\begin{array}{l}\text { Sporer, } 1996 \\
\text { Retrospective cohort }\end{array}$ & Not reported & No adjustment performed & $\begin{array}{l}\text { A vs. B: } \\
\text { Response rate (GCS } \geq 14 \text { and } \\
\text { RR } \geq 10 / \text { minute within } 5 \text { minutes of } \\
\text { naloxone administration): } 488 / 518 \text { ( } 94 \%) \\
\text { vs. } 69 / 73 \text { (95\%), RR } 1.0 \text { (95\% Cl } 0.94 \text { to } \\
1.1)\end{array}$ & CDC grant & $\begin{array}{l}\text { Data on hospital transport, but } \\
\text { does not include data on those } \\
\text { not transported. Study done in } \\
\text { San Francisco, CA, USA. Race } \\
\text { data unknown in a large number } \\
\text { of patients in the study. } \\
\text { Response defined as } \\
\text { improvement to a GCS } \\
\geq 14 \text { and RR +1- within } 5 \\
\text { minutes of naloxone } \\
\text { administration. } 52 \text { patients } \\
\text { received both IM and IV with a } \\
\text { response rate of } 98 \% \text {. Not clear } \\
\text { why this group got both, authors } \\
\text { presume use of a larger dose of } \\
\text { opioid. Most common routes of } \\
\text { use for opioid were IV and } \\
\text { unknown. Formulation of } \\
\text { naloxone not specified but } \\
\text { presumably same one used for } \\
\text { IV and IM administration. }\end{array}$ \\
\hline $\begin{array}{l}\text { Wanger, } 1998 \\
\text { Prospective cohort with } \\
\text { historical control }\end{array}$ & Hospital discharge & No adjustment performed & $\begin{array}{l}\text { Time interval from drug administration to } \\
\text { respiratory rate } \geq 10 \text { breaths per minute: } \\
5.5 \text { vs. } 3.8 \text { minutes, } p=0.001 \\
\text { Time interval from arrival at patient's side } \\
\text { to respiratory rate } \geq 10 \text { breaths per } \\
\text { minute: } \\
9.6 \text { vs. } 9.3 \text { minutes, } p=0.67 \\
\text { Proportion requiring multiple naloxone } \\
\text { doses: } 15 \% \text { (18/122) vs. } 35 \%(26 / 74) \text {, } \\
\text { RR } \\
0.42(95 \% \mathrm{Cl} 0.25 \text { to } 0.71)\end{array}$ & $\begin{array}{l}\text { Medical } \\
\text { Research } \\
\text { Council/ } \\
\text { University } \\
\text { of British } \\
\text { Columbia }\end{array}$ & $\begin{array}{l}\text { No comment on formulation but } \\
\text { presumably the same was use } \\
\text { for IV and SC. The } \\
\text { administration of medications is } \\
\text { usually restricted to paramedics } \\
\text { but this was not specified clearly } \\
\text { in the paper. Similar } \\
\text { improvement in RR related to } \\
\text { time required to establish IV. } \\
\text { Study conducted in city of } \\
\text { Vancouver, BC, Canada. }\end{array}$ \\
\hline
\end{tabular}

ALS = advance life support; CI = confidence interval; EMS = emergency medical services; GCS = Glasgow Coma Scale; IN = intranasal; IV = intravenous; KQ = Key Question; RR = relative risk; $\mathrm{SQ}=$ subcutaneous 
Table D-3. Key Question 4: Data abstraction of observational studies

\begin{tabular}{|c|c|c|c|c|c|}
\hline $\begin{array}{l}\text { Author, Year } \\
\text { Study Design }\end{array}$ & Key Question & $\begin{array}{l}\text { Country Setting } \\
\text { Study Years }\end{array}$ & Eligibility Criteria & $\begin{array}{l}\text { Intervention Characteristics by } \\
\text { Comparison Group } \\
\text { (dose, duration and route of } \\
\text { treatment; training/background of } \\
\text { personnel administering drug; timing } \\
\text { of administration) }\end{array}$ & $\begin{array}{l}\text { Screened } \\
\text { Eligible } \\
\text { Enrolled } \\
\text { Analyzed } \\
\text { Loss to Followup }\end{array}$ \\
\hline $\begin{array}{l}\text { Boyd, } 2006 \\
\text { Retrospective cohort }\end{array}$ & \begin{tabular}{|l|} 
KQ4 \\
Transport
\end{tabular} & \begin{tabular}{|l|} 
Finland \\
Out-of-hospital \\
$1995-2000$
\end{tabular} & $\begin{array}{l}\text { Patients with signs and symptoms } \\
\text { of } \\
\text { opioid overdose: } \\
\text { Respirations }<12 \text { breaths/minute } \\
\text { Peripheral oxygen saturation }<90 \% \\
\text { without supplemental oxygen or } \\
<95 \% \text { with oxygen Cyanosis on } \\
\text { arrival of first responding unit } \\
\text { GCS } \leq 8 \text { and witnessed using } \\
\text { heroin or with circumstantial } \\
\text { evidence of drug use } \\
\text { Excluded patients with cardiac } \\
\text { arrest, polydrug or alcohol use, or } \\
\text { use of opioids other than heroin }\end{array}$ & $\begin{array}{l}\text { Refused transport to emergency } \\
\text { department }(n=84)\end{array}$ & $\begin{array}{l}\text { Screened: } 269 \\
\text { Eligible: } 162 \\
\text { Enrolled: } 163 \text { (79 vs. } 84) \\
\text { Analyzed: } 145 \text { (61 vs. } 84) \\
\text { Lost to followup: } 18 \text { (18 vs. 0) }\end{array}$ \\
\hline $\begin{array}{l}\text { Levine, } 2016 \\
\text { Retrospective Cohort }\end{array}$ & \begin{tabular}{|l|} 
KQ4 \\
Transport
\end{tabular} & \begin{tabular}{|l|} 
United States \\
Out-of-hospital \\
$2011-2013$
\end{tabular} & $\begin{array}{l}\text { Patients who had a respiratory rate } \\
\text { lower than } 12 \text { who received } \\
\text { naloxone and who signed out } \\
\text { against medical advice. } \\
\text { Excluded patients who were } \\
\text { transported to the hospital. }\end{array}$ & $\begin{array}{l}\text { Refused transport to emergency } \\
\text { department }(n=205)\end{array}$ & $\begin{array}{l}\text { Screened }=\text { NR } \\
\text { Eligible = 205 } \\
\text { Enrolled }=205 \\
\text { Analyzed }=205\end{array}$ \\
\hline
\end{tabular}




\begin{tabular}{|c|c|c|c|c|c|}
\hline $\begin{array}{l}\text { Author, Year } \\
\text { Study Design }\end{array}$ & $\begin{array}{l}\text { Population Characteristics (age, } \\
\text { sex, race, psychiatric } \\
\text { comorbidities, prior overdose } \\
\text { episodes, receipt of out-of- } \\
\text { hospital naloxone, response to } \\
\text { out-of-hospital naloxone) }\end{array}$ & $\begin{array}{l}\text { Exposure Characteristics (type of opioid } \\
\text { involve, dose of opioid involved, other } \\
\text { contributing drugs, time since overdose, } \\
\text { naloxone dosing) }\end{array}$ & $\begin{array}{l}\text { Method for } \\
\text { Assigning } \\
\text { Exposures }\end{array}$ & $\begin{array}{l}\text { Method For } \\
\text { Assessing } \\
\text { Outcomes and } \\
\text { Confounders }\end{array}$ & $\begin{array}{l}\text { Duration of } \\
\text { Followup }\end{array}$ \\
\hline $\begin{array}{l}\text { Boyd, } 2006 \\
\text { Retrospective cohort }\end{array}$ & $\begin{array}{l}\text { Overall population characteristics } \\
\text { (not reported by intervention group) } \\
\text { Age: } 26 \text { years } \\
\text { Male: } 83 \% \\
\text { Race: Not reported } \\
\text { Psychiatric comorbidities: Not } \\
\text { reported } \\
\text { Prior overdose episodes: Not } \\
\text { reported } \\
\text { Received out-of-hospital naloxone: } \\
85 \%\end{array}$ & $\begin{array}{l}\text { Type of opioid: Heroin only } \\
\text { Dose of opioid: IV: } 43 / 145(29.7 \%) \\
\text { Sniffed: } 3 / 145(2.2 \%) \\
\text { Smoked: } 2 / 145(1.4 \%) \text { Unreported: } 97 / 145 \\
\text { (66.9\%) } \\
\text { Other contributing drugs: None suspected } \\
\text { Time since overdose: Not reported } \\
\text { Naloxone dosing, not reported by comparison } \\
\text { group: } \\
\text { Naloxone dose } \leq 0.4 \mathrm{mg}: 87 / 123(70.7 \%) \\
\text { Naloxone dose } 0.4-0.8 \mathrm{mg}: 36 / 123(29.3 \%) \\
\text { Prehospital route of administration: } \\
\text { IV: } 46 / 123(37.4 \%) \text { IM/SQ: } 35 / 123(28.5 \%) \\
\text { IV + IM/SQ: } 33 / 123(26.8 \%) \text { Not reported: } 9 / 123 \\
\text { (7.3\%) }\end{array}$ & EMS records & $\begin{array}{l}\text { EMS and hospital } \\
\text { records for } \\
\text { outcomes; } \\
\text { confounders not } \\
\text { assessed }\end{array}$ & $\begin{array}{l}12 \text { hours for patients } \\
\text { who refused } \\
\text { transport }\end{array}$ \\
\hline $\begin{array}{l}\text { Levine, } 2016 \\
\text { Retrospective } \\
\text { Cohort }\end{array}$ & $\begin{array}{l}\text { Age } 41 \text { years }(29-53) \\
\text { Male } 87 \% \text { Race = NR } \\
\text { Psychiatric Comorbidities= NR Prior } \\
\text { Overdoses = NR }\end{array}$ & $\begin{array}{l}\text { Type of Opioid: Opioid toxicity } \\
\text { Dose = NR Route = NR } \\
\text { Other contributing drugs = NR Time since } \\
\text { overdose = NR } \\
\text { Naloxone dosing } \\
\text { When a patient is suspected of opiate toxicity, } \\
\text { there are standing orders permitting the } \\
\text { paramedic to administer } 0.8-2 \mathrm{mg} \text { of naloxone } \\
\text { intramuscularly, or intranasally prior to } \\
\text { establishing intravenous access. The dose can } \\
\text { be repeated if needed. } \\
\text { Exact Doses NR } \\
\text { Route = NR per patient }\end{array}$ & EMS Records & $\begin{array}{l}\text { EMS and medical } \\
\text { examiners records } \\
\text { for outcomes; } \\
\text { confounders not } \\
\text { assessed }\end{array}$ & $\begin{array}{l}24 \text { hours, } 30 \text { days } \\
\text { and } 6 \text { months }\end{array}$ \\
\hline
\end{tabular}




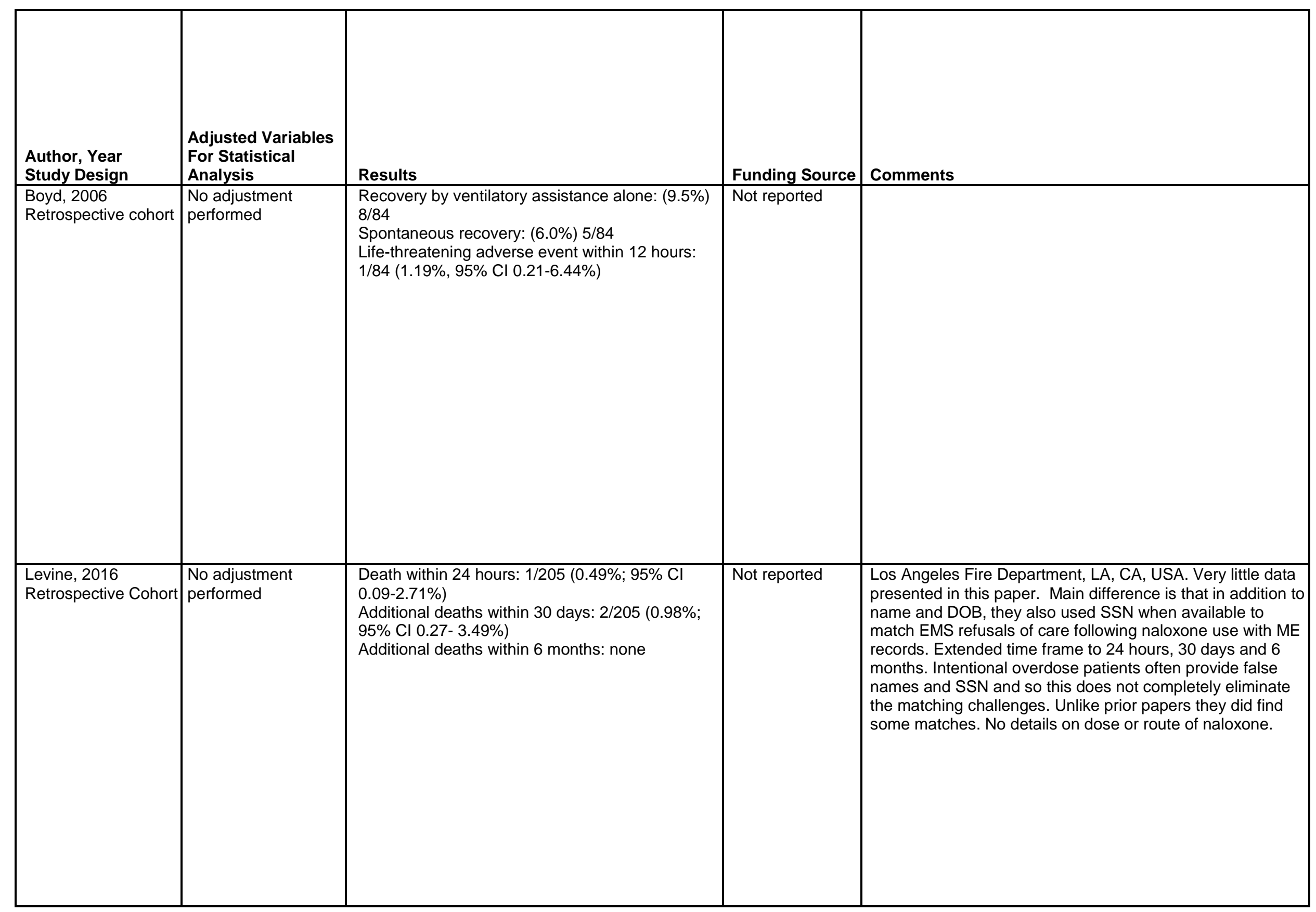




\begin{tabular}{|c|c|c|c|c|c|}
\hline $\begin{array}{l}\text { Author, Year } \\
\text { Study Design }\end{array}$ & Key Question & $\begin{array}{l}\text { Country Setting } \\
\text { Study Years }\end{array}$ & Eligibility Criteria & $\begin{array}{l}\text { Intervention Characteristics by } \\
\text { Comparison Group } \\
\text { (dose, duration and route of } \\
\text { treatment; training/background of } \\
\text { personnel administering drug; } \\
\text { timing of administration) }\end{array}$ & $\begin{array}{l}\text { Screened } \\
\text { Eligible } \\
\text { Enrolled } \\
\text { Analyzed } \\
\text { Loss to Followup }\end{array}$ \\
\hline $\begin{array}{l}\text { Rudolph, } 2011 \\
\text { Retrospective cohort }\end{array}$ & \begin{tabular}{|l|} 
KQ4 \\
Transport
\end{tabular} & $\begin{array}{l}\text { The Netherland } \\
\text { Out-of-hospital } \\
\text { 1994-2003 }\end{array}$ & $\begin{array}{l}\text { Patients treated by physician-based } \\
\text { medical emergency unit for opioid } \\
\text { overdose and not transferred to } \\
\text { hospital. } \\
\text { Patients who were transported to } \\
\text { hospital were excluded. }\end{array}$ & Not transported to hospital $(n=2241)$ & $\begin{array}{l}\text { Screened: } 4762 \\
\text { Eligible: } 2241 \\
\text { Enrolled: } 2241 \\
\text { Analyzed: } 2241\end{array}$ \\
\hline $\begin{array}{l}\text { Vilke, } 1999 \\
\text { Retrospective Cohort }\end{array}$ & $\begin{array}{l}\text { KQ4 } \\
\text { Transport }\end{array}$ & $\begin{array}{l}\text { United States } \\
\text { Out-of-hospital } \\
1996\end{array}$ & $\begin{array}{l}\text { Patients who received naloxone } \\
\text { and were released against medical } \\
\text { advice. } \\
\text { Patients who were transported } \\
\text { were excluded. }\end{array}$ & $\begin{array}{l}\text { Refused transport to emergency } \\
\text { department }(n=317)\end{array}$ & $\begin{array}{l}\text { Screened = } 1714 \\
\text { Eligible = } 317 \\
\text { Enrolled }=317 \\
\text { Analyzed }=317\end{array}$ \\
\hline
\end{tabular}




\begin{tabular}{|c|c|c|c|c|c|}
\hline $\begin{array}{l}\text { Author, Year } \\
\text { Study Design }\end{array}$ & $\begin{array}{l}\text { Population Characteristics (age, } \\
\text { sex, race, psychiatric } \\
\text { comorbidities, prior overdose } \\
\text { episodes, receipt of out-of- } \\
\text { hospital naloxone, response to } \\
\text { out-of-hospital naloxone) }\end{array}$ & $\begin{array}{l}\text { Exposure Characteristics (type of opioid } \\
\text { involve, dose of opioid involved, other } \\
\text { contributing drugs, time since overdose, } \\
\text { naloxone dosing) }\end{array}$ & $\begin{array}{l}\text { Method for } \\
\text { Assigning } \\
\text { Exposures }\end{array}$ & $\begin{array}{l}\text { Method For } \\
\text { Assessing } \\
\text { Outcomes and } \\
\text { Confounders } \\
\end{array}$ & $\begin{array}{l}\text { Duration of } \\
\text { Followup }\end{array}$ \\
\hline $\begin{array}{l}\text { Rudolph, } 2011 \\
\text { Retrospective cohort }\end{array}$ & $\begin{array}{l}\text { Age, sex, race: Not reported } \\
\text { Type of Opioid, Dose, Route: Not } \\
\text { reported }\end{array}$ & $\begin{array}{l}\text { Type of opioid: Not reported } \\
\text { Dose of opioid: Not reported } \\
\text { Other contributing drugs: Not reported } \\
\text { Time since overdose: Not reported } \\
\text { Naloxone dosing: } \\
\text { Intravenous } 0.8 \mathrm{mg} \text {, supplemented with } 0.4 \mathrm{mg} \\
\text { intramuscular or subqutaneous at physician's } \\
\text { discretion; naloxone titrated to effect. Exact } \\
\text { doses not reported. }\end{array}$ & $\begin{array}{l}\text { EMS and medical } \\
\text { examiner records } \\
\text { for outcomes }\end{array}$ & $\begin{array}{l}\text { EMS and medical } \\
\text { examiner records for } \\
\text { outcomes }\end{array}$ & 48 hours \\
\hline \begin{tabular}{l|} 
Vilke, 1999 \\
Retrospective Cohort
\end{tabular} & $\begin{array}{l}\text { Age } 37 \text { Years }(18-79) \\
\text { Male } 82.7 \% \text { Race }=\text { NR } \\
\text { Psychiatric Comorbidities = NR Prior } \\
\text { Overdoses = NR }\end{array}$ & $\begin{array}{l}\text { Type of Opioid: Suspected heroin and opiate } \\
\text { overdose Dose = NR Route = NR } \\
\text { Other contributing drugs = NR Time since } \\
\text { overdose = NR } \\
\text { Naloxone Dosing } \\
\text { Protocols give medics the authority to treat with } \\
2 \mathrm{mg} \text { naloxone IV/IM or } 4 \mathrm{mg} \text { via endotracheal } \\
\text { tube and may repeat the dose if no response. } \\
\text { Dose }=59(18.6 \%) \text { single dose } \\
\text { Route }=41(69.5 \%) \text { IV } 18(30.5 \%) \text { IM Two Doses } \\
=249(78.6 \%) \\
\text { IV- IM }=180 \\
\text { IM }- \text { IV = 28 } \\
\text { IM }- \text { IM = } 11 \\
\text { IV - IV = 30 } \\
\text { Three Doses = } 9(2.8 \%) \text { IV-IV-IM = 7 } \\
\text { IM-IM-IM = } 1 \\
\text { IV-IM-IM = } 1\end{array}$ & EMS Records & $\begin{array}{l}\text { EMS and medical } \\
\text { examiners records } \\
\text { for outcomes; } \\
\text { confounders not } \\
\text { assessed }\end{array}$ & 12 hours \\
\hline
\end{tabular}




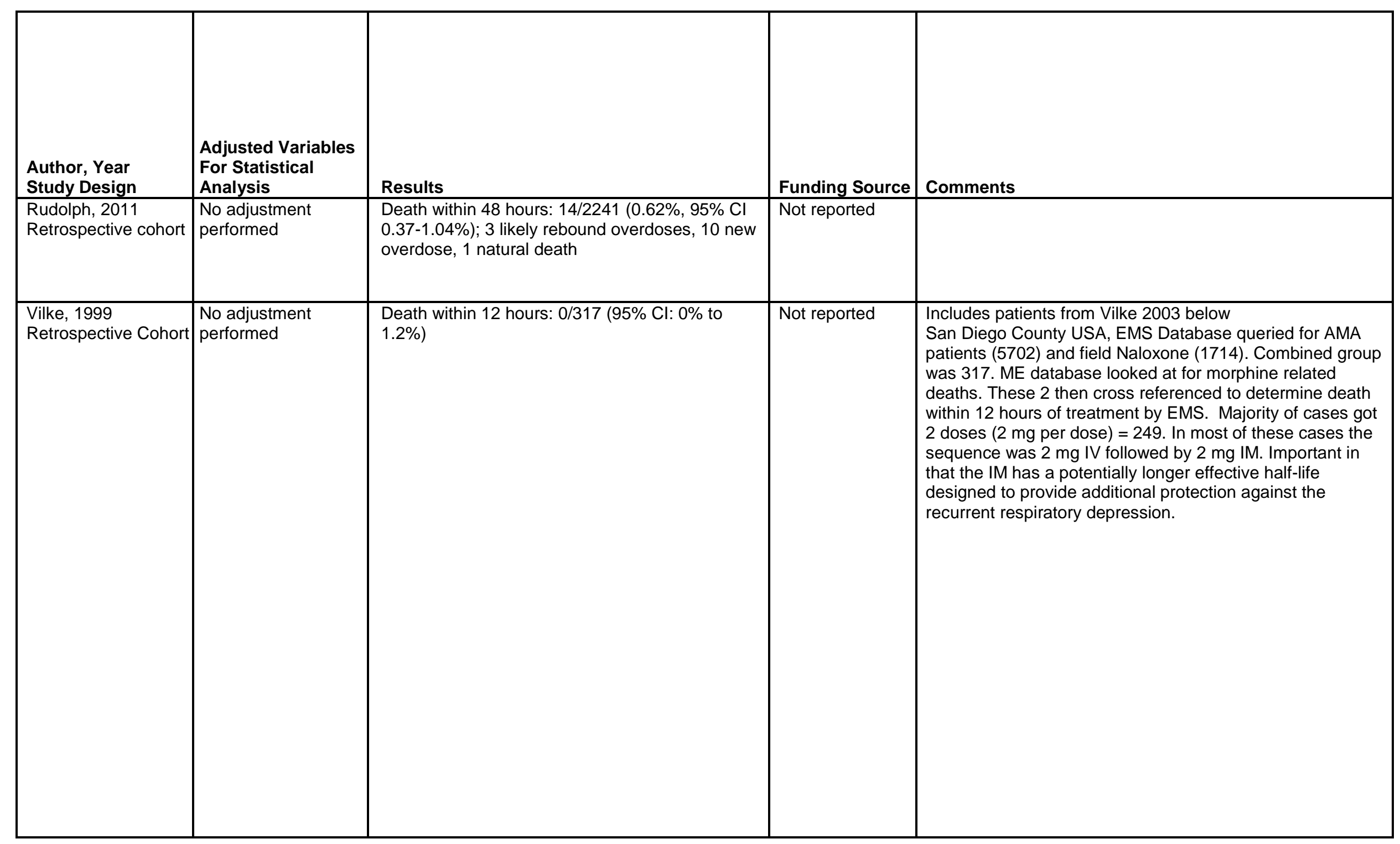




\begin{tabular}{|c|c|c|c|c|c|}
\hline $\begin{array}{l}\text { Author, Year } \\
\text { Study Design }\end{array}$ & Key Question & $\begin{array}{l}\text { Country Setting } \\
\text { Study Years } \\
\end{array}$ & Eligibility Criteria & $\begin{array}{l}\text { Intervention Characteristics by } \\
\text { Comparison Group } \\
\text { (dose, duration and route of } \\
\text { treatment; training/background of } \\
\text { personnel administering drug; } \\
\text { timing of administration) }\end{array}$ & $\begin{array}{l}\text { Screened } \\
\text { Eligible } \\
\text { Enrolled } \\
\text { Analyzed } \\
\text { Loss to Followup }\end{array}$ \\
\hline $\begin{array}{l}\text { Vilke, } 2003 \\
\text { Retrospective } \\
\text { Cohort }\end{array}$ & \begin{tabular}{|l|} 
KQ4 \\
Transport
\end{tabular} & \begin{tabular}{|l|} 
United States \\
Out-of-hospital \\
$1996-2000$
\end{tabular} & $\begin{array}{l}\text { Patients who received naloxone } \\
\text { and were released AMA. } \\
\text { Patients who were transported } \\
\text { were excluded. }\end{array}$ & $\begin{array}{l}\text { Refused transport to emergency } \\
\text { department }(n=998)\end{array}$ & $\begin{array}{l}\text { Screened }=8366 \\
\text { Eligible }=998 \\
\text { Enrolled }=998 \\
\text { Analyzed }=998\end{array}$ \\
\hline
\end{tabular}




\begin{tabular}{|c|c|c|c|c|c|}
\hline $\begin{array}{l}\text { Author, Year } \\
\text { Study Design }\end{array}$ & $\begin{array}{l}\text { Population Characteristics (age, } \\
\text { sex, race, psychiatric } \\
\text { comorbidities, prior overdose } \\
\text { episodes, receipt of out-of- } \\
\text { hospital naloxone, response to } \\
\text { out-of-hospital naloxone) }\end{array}$ & $\begin{array}{l}\text { Exposure Characteristics (type of opioid } \\
\text { involve, dose of opioid involved, other } \\
\text { contributing drugs, time since overdose, } \\
\text { naloxone dosing) }\end{array}$ & $\begin{array}{l}\text { Method for } \\
\text { Assigning } \\
\text { Exposures }\end{array}$ & $\begin{array}{l}\text { Method For } \\
\text { Assessing } \\
\text { Outcomes and } \\
\text { Confounders } \\
\end{array}$ & $\begin{array}{l}\text { Duration of } \\
\text { Followup }\end{array}$ \\
\hline $\begin{array}{l}\text { Vilke, } 2003 \\
\text { Retrospective } \\
\text { Cohort }\end{array}$ & $\begin{array}{l}\text { Age } 37.7 \text { years }(16-83) \\
\text { Male } 83.8 \% \text { Race }=\text { NR } \\
\text { Psychiatric Comorbidities = NR } \\
\text { Prior Overdoses = NR }\end{array}$ & $\begin{array}{l}\text { Type of Opioid: Suspected heroin and opiate } \\
\text { overdose Dose = NR Route = NR } \\
\text { Other contributing drugs = NR Time since } \\
\text { overdose = NR } \\
\text { Naloxone Dosing } \\
\text { Protocols give medics the authority to treat with } \\
2 \mathrm{mg} \text { naloxone IV/IM or } 4 \mathrm{mg} \text { via endotracheal } \\
\text { tube and may repeat the dose if no response. } \\
\text { Dose = } 260(26.1 \%) \text { single dose } \\
\text { Route }=179(68.6 \%) \text { IV } 80(30.8 \%) \text { IM Two } \\
\text { Doses }=714(71.5 \%) \\
\text { IV- IM }=552 \\
\text { IM }- \text { IV }=40 \\
\text { IM }- \text { IM }=47 \\
\text { IV }- \text { IV }=74 \\
\text { IM }-?=1 \\
\text { Three Doses = } 24(2.4 \%) \text { IV-IV-IM = } 16 \\
\text { IM-IM-IM = } \\
\text { IV-IM-IM = } 3 \\
\text { IV-IV-IV = } 2 \\
\text { IM-IV-IM = } 1 \\
\text { IM-IV-IV = 1 }\end{array}$ & EMS Records & $\begin{array}{l}\text { EMS and medical } \\
\text { examiners records } \\
\text { for outcomes; } \\
\text { confounders not } \\
\text { assessed }\end{array}$ & 12 hours \\
\hline
\end{tabular}




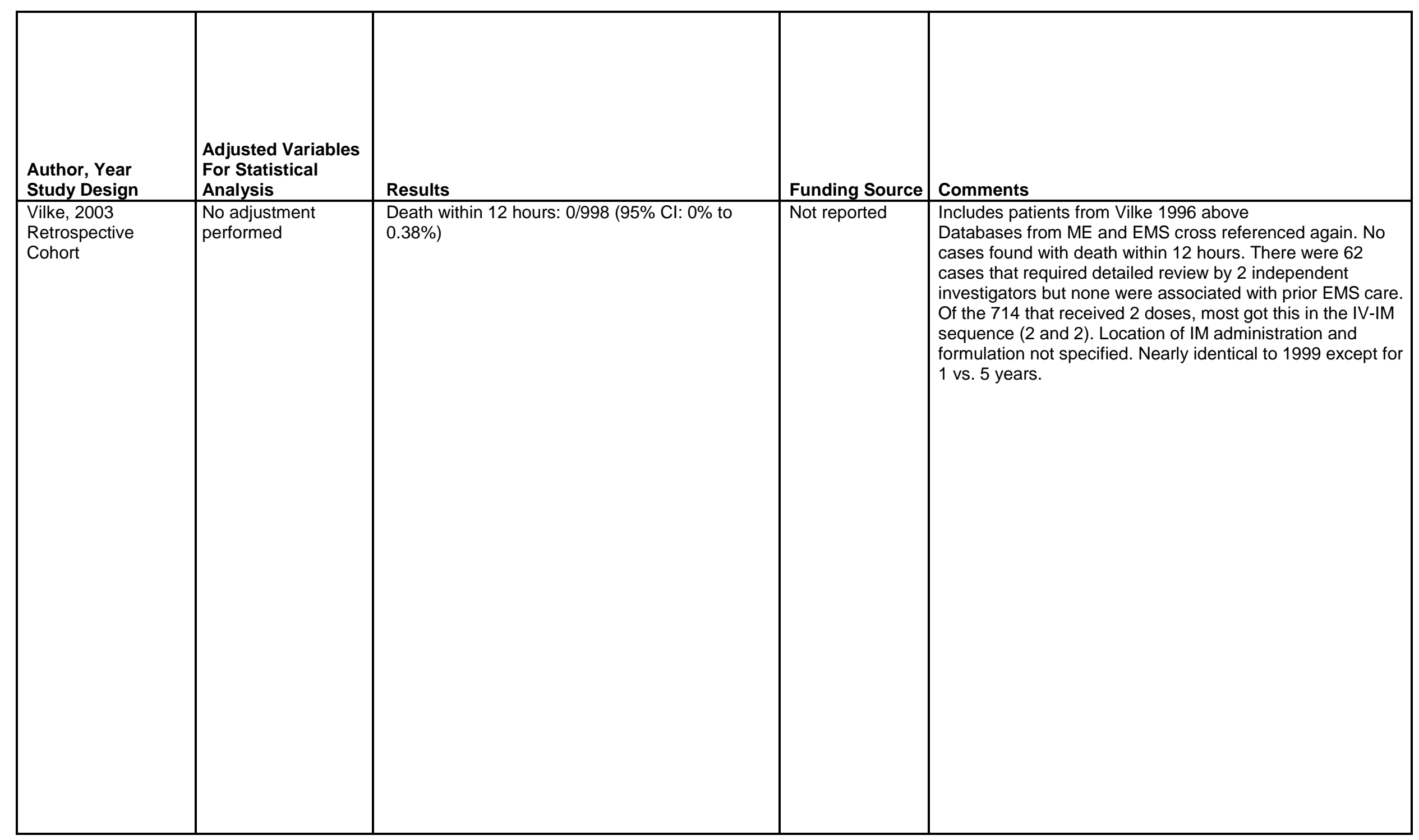




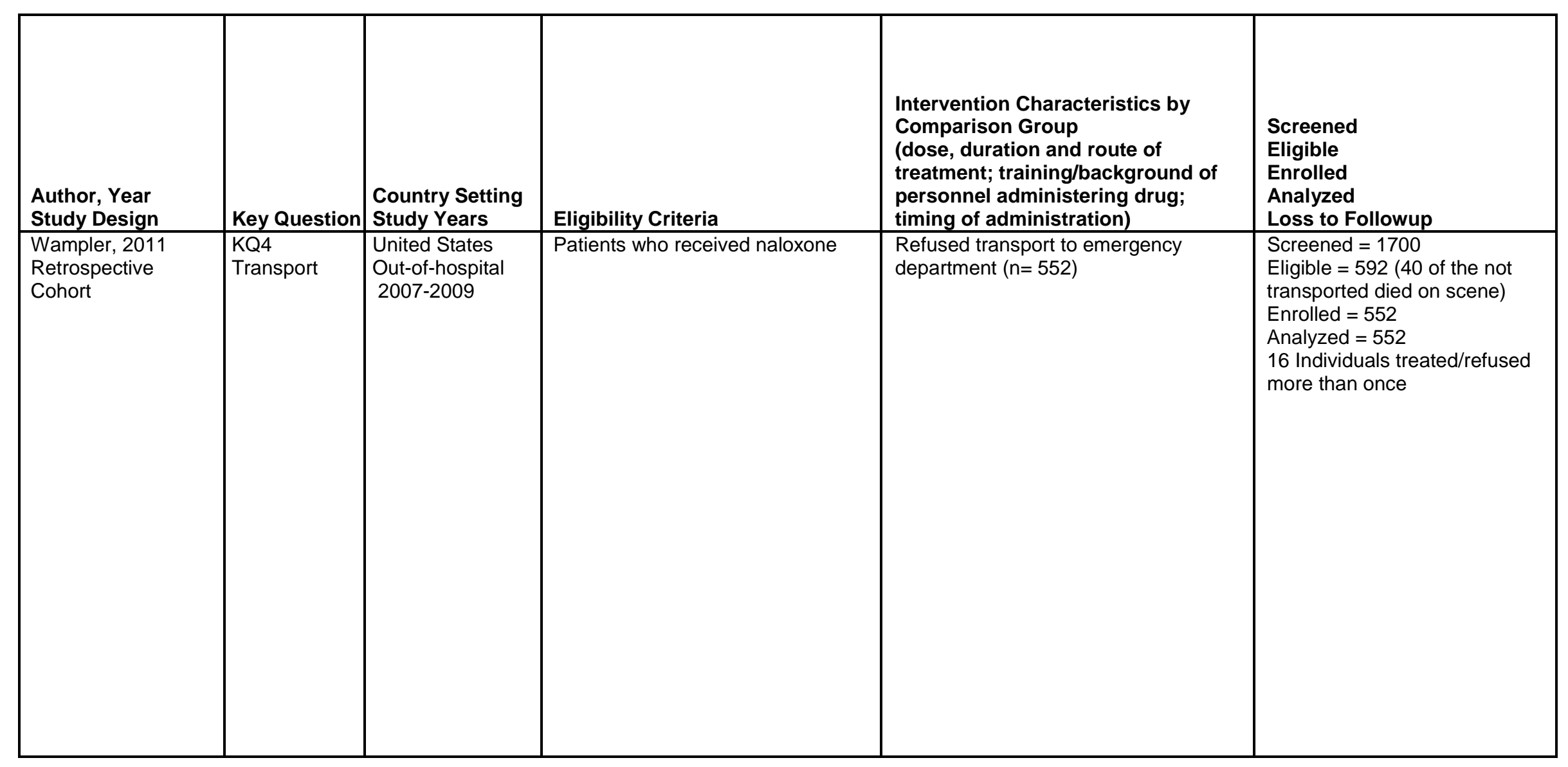




\begin{tabular}{|c|c|c|c|c|c|}
\hline $\begin{array}{l}\text { Author, Year } \\
\text { Study Design }\end{array}$ & $\begin{array}{l}\text { Population Characteristics (age, } \\
\text { sex, race, psychiatric } \\
\text { comorbidities, prior overdose } \\
\text { episodes, receipt of out-of- } \\
\text { hospital naloxone, response to } \\
\text { out-of-hospital naloxone) }\end{array}$ & $\begin{array}{l}\text { Exposure Characteristics (type of opioid } \\
\text { involve, dose of opioid involved, other } \\
\text { contributing drugs, time since overdose, } \\
\text { naloxone dosing) }\end{array}$ & $\begin{array}{l}\text { Method for } \\
\text { Assigning } \\
\text { Exposures }\end{array}$ & $\begin{array}{l}\text { Method For } \\
\text { Assessing } \\
\text { Outcomes and } \\
\text { Confounders }\end{array}$ & $\begin{array}{l}\text { Duration of } \\
\text { Followup }\end{array}$ \\
\hline $\begin{array}{l}\text { Wampler, } 2011 \\
\text { Retrospective } \\
\text { Cohort }\end{array}$ & $\begin{array}{l}\text { Age } 38 \pm 15 \text { years }(13-91) \\
\text { Male } 72 \% \text { Race }=\text { NR } \\
\text { Psychiatric Comorbidities }=\text { NR } \\
\text { Prior Overdoses }=\text { NR }\end{array}$ & $\begin{array}{l}\text { Type of opioid: Apparent or suspected narcotic } \\
\text { overdose Dose = NR Route = NR } \\
\text { Other contributing drugs = NR Time since } \\
\text { overdose = NR } \\
\text { Naloxone Dosing } \\
\text { Dose and Route = } 2 \mathrm{mg} \text { IM with an additional } 2 \\
\text { mg IV infusion. } \\
\text { A third dose }(2 \mathrm{mg} \mathrm{IM}) \text { is given with medical } \\
\text { consultation }\end{array}$ & EMS Records & $\begin{array}{l}\text { EMS and medical } \\
\text { examiners records } \\
\text { for outcomes; } \\
\text { confounders not } \\
\text { assessed }\end{array}$ & $\begin{array}{l}48 \text { hours, } 30 \text { days, } \\
\text { and up to } 372 \text { days }\end{array}$ \\
\hline
\end{tabular}




\begin{tabular}{|c|c|c|c|c|}
\hline $\begin{array}{l}\text { Author, Year } \\
\text { Study Design }\end{array}$ & $\begin{array}{l}\text { Adjusted Variables } \\
\text { For Statistical } \\
\text { Analysis }\end{array}$ & Results & Funding Source & Comments \\
\hline $\begin{array}{l}\text { Wampler, } 2011 \\
\text { Retrospective } \\
\text { Cohort }\end{array}$ & $\begin{array}{l}\text { No adjustment } \\
\text { Performed }\end{array}$ & $\begin{array}{l}\text { Deaths within } 48 \text { hours: } 0 / 5520 \%, 95 \% \mathrm{Cl} 0 \% \text { to } \\
0.69 \%) \\
\text { Deaths in field prior to transport: } 40 / 552(7.2 \%) \\
\text { None of the patients were examined within the } 2 \\
\text { day endpoint. } \\
\text { Deaths from } 4-372 \text { days: } 9 / 592(1.6 \%, 95 \% \mathrm{Cl} \\
0.5 \% \text { to } 2.7 \%)\end{array}$ & $\begin{array}{l}\text { Office of the } \\
\text { Medical Director } \\
\text { for the San } \\
\text { Antonio } \\
\text { Department of } \\
\text { Emergency } \\
\text { Medicine }\end{array}$ & $\begin{array}{l}\text { All patients receiving Naloxone included. } 20 \text { month study } \\
\text { period Nov } 2997 \text { to June } 2009.1,700 \text { cases with naloxone } \\
\text { administration. } 592 \text { nontransported. } 40 \text { of these were } \\
\text { cardiac arrest that had field termination of resuscitation } \\
\text { leaving } 552 \text { with naloxone administration and against } \\
\text { medical advice release. Cross referenced EMS cases with } \\
\text { ME cases. No deaths within } 48 \text { hours, } 2 \text { deaths within } 30 \\
\text { days (one from heroin+cocaine OD on day } 4 \text {, other from } \\
\text { gunshot wound) During the } 20 \text { month study period (600 } \\
\text { days or so), total of } 9 \text { deaths cross referenced. Average } \\
\text { time from EMS presentation to ME office death was } 170 \\
\text { days, range } 4-372 \text {. Some of the nontransported cases were } \\
\text { released to law enforcement officer as were some of the } \\
\text { presumed deaths as outline in Table } 1 \text { and } 2 \text {. Limitation as } \\
\text { with other ME cross reference studies are that age and } \\
\text { DOB can be inaccurate due to falsification. There dosing } \\
\text { protocol is also aggressive } 2 \text { mg IM, followed by } 2 \text { mg IV } \\
\text { and then if the patient agrees to it another } 2 \text { mg IM prior to } \\
\text { signing against medical advice. Results like Vilke but } \\
\text { looking at } 48 \text { hours, } 30 \text { day and then } 20 \text { months. }\end{array}$ \\
\hline
\end{tabular}

examiner; $\mathrm{NR}$ = not reported; $\mathrm{OD}$ = overdose; $\mathrm{SQ}$ = subcutaneous; $\mathrm{SSN}$ = social security number 


\section{Appendix E. Risk of Bias}

Table E-1. Key Question 1: Risk of bias ratings of randomized controlled trials

\begin{tabular}{|c|c|c|c|c|c|c|c|}
\hline Author, Year & $\begin{array}{l}\text { Randomization } \\
\text { adequate? }\end{array}$ & $\begin{array}{l}\text { Allocation } \\
\text { concealment } \\
\text { adequate? }\end{array}$ & $\begin{array}{l}\text { Groups similar at } \\
\text { baseline? }\end{array}$ & $\begin{array}{l}\text { Eligibility criteria } \\
\text { specified? }\end{array}$ & $\begin{array}{l}\text { Outcome assessors } \\
\text { masked? }\end{array}$ & $\begin{array}{l}\text { Care provider } \\
\text { masked? }\end{array}$ & Patient masked? \\
\hline Kerr, 2009 & Yes & Yes & No & Yes & No & No & No \\
\hline Kelly, 2005 & Method NR & Yes & $\begin{array}{l}\text { Yes, There were } \\
\text { some differences in } \\
\text { setting but I don't think } \\
\text { these were major or } \\
\text { should impact the } \\
\text { results }\end{array}$ & Yes & No & No & No \\
\hline Sabzghabaee, 2014 & Yes & Unclear & $\begin{array}{l}\text { Some differences in the } \\
\text { type of opioid. }\end{array}$ & Yes & Unclear & Unclear & Unclear \\
\hline
\end{tabular}




\begin{tabular}{|c|c|c|c|c|c|c|c|}
\hline Kerr, 2009 & Yes & No & Yes & Yes (3.4\%) & Yes & Government & Medium \\
\hline Kelly, 2005 & Yes & No & Yes & Yes (14.9\%) & Yes & Grant & Medium \\
\hline Sabzghabaee, 2014 & No & Unclear & Unclear & \begin{tabular}{|l} 
Unclear, but states \\
there were \\
exclusions
\end{tabular} & Yes & University & High \\
\hline
\end{tabular}

$\mathrm{NR}=$ not reported 
Table E-2. Key Question 1: Risk of bias ratings of observational studies

\begin{tabular}{|c|c|c|c|c|c|}
\hline Author, Year & $\begin{array}{l}\text { Did the study attempt } \\
\text { to enroll a random } \\
\text { sample or } \\
\text { consecutive patients } \\
\text { meeting inclusion } \\
\text { criteria (inception } \\
\text { cohort)? }\end{array}$ & $\begin{array}{l}\text { Were the groups } \\
\text { comparable at baseline? }\end{array}$ & $\begin{array}{l}\text { Did the study use } \\
\text { accurate methods for } \\
\text { ascertaining exposures, } \\
\text { potential confounders, } \\
\text { and outcomes? }\end{array}$ & $\begin{array}{l}\text { Were outcome assessors } \\
\text { andlor data analysts } \\
\text { blinded to treatment? }\end{array}$ & $\begin{array}{l}\text { Did the article report } \\
\text { attrition and missing data? }\end{array}$ \\
\hline Merlin, 2010 & Yes & $\begin{array}{l}\text { No. Differences in } \\
\text { congestion evidence and } \\
\text { naloxone dose given }\end{array}$ & Yes & No & Yes \\
\hline Robertson, 2009 & Unclear & No & Yes & No & Unclear \\
\hline Sporer, 1996 & Yes & Unclear & Yes & No & No \\
\hline Wanger, 1998 & Yes & Yes & Yes & No & Yes \\
\hline
\end{tabular}




\begin{tabular}{|c|c|c|c|c|c|}
\hline Author, Year & $\begin{array}{l}\text { Did the study perform } \\
\text { appropriate statistical } \\
\text { analyses on potential } \\
\text { confounders? }\end{array}$ & $\begin{array}{l}\text { Is there important } \\
\text { differential attrition or } \\
\text { missing data or overall high } \\
\text { attrition or missing data? }\end{array}$ & $\begin{array}{l}\text { Were outcomes } \\
\text { prespecified and defined, } \\
\text { and ascertained using } \\
\text { accurate methods? }\end{array}$ & Risk of Bias & Comments \\
\hline Merlin, 2010 & No & No & Yes & High & $\begin{array}{l}\text { Analysis restricted to patients with } \\
\text { "confirmed opioid overdose" } \\
\text { (criteria not specified) }\end{array}$ \\
\hline Robertson, 2009 & No & Unclear & Yes & High & \\
\hline Sporer, 1996 & No & No & Yes & High & $\begin{array}{l}\text { Analysis restricted to patients who } \\
\text { met criteria for presumed opioid } \\
\text { overdose }\end{array}$ \\
\hline Wanger, 1998 & No & No & Yes & High & $\begin{array}{l}\text { Excluded } 25 \text { patients due to } \\
\text { inappropriate use of naloxone } \\
\text { protocol (respiratory rate } \geq 10 \\
\text { breaths/minute) }\end{array}$ \\
\hline
\end{tabular}


Table E-3. Key Question 4: Risk of bias ratings of observational studies

\begin{tabular}{|l|l|l|l|l|}
\hline & & & \\
& $\begin{array}{l}\text { Did the study attempt to } \\
\text { enroll a random sample or } \\
\text { consecutive patients } \\
\text { meeting inclusion criteria } \\
\text { (inception cohort)? }\end{array}$ & $\begin{array}{l}\text { Were the groups } \\
\text { comparable at baseline? }\end{array}$ & $\begin{array}{l}\text { Did the study use accurate } \\
\text { methods for ascertaining } \\
\text { exposures, potential confounders, } \\
\text { and outcomes? }\end{array}$ & $\begin{array}{l}\text { Were outcome assessors and/or } \\
\text { data analysts blinded to } \\
\text { treatment? }\end{array}$ \\
Author, Year & Yes & Unclear & Yes & No \\
\hline Boyd, 2006 & Yes & NA (single-arm cohort) & Yes & Unclear \\
\hline Levine, 2016 & Yes & NA (single-arm cohort) & Yes & No \\
\hline Rudolph, 2011 & Yes & NA (single-arm cohort) & Yes & Unclear \\
\hline Vilke, 1999 & NA (single-arm cohort) & Yes & Unclear \\
\hline Vilke, 2003 & Yes & NA (single-arm cohort) & Yes & Unclear \\
\hline Wampler, 2011 & Yes & & \\
\hline
\end{tabular}




\begin{tabular}{|c|c|c|c|c|c|}
\hline Author, Year & $\begin{array}{l}\text { Did the article report attrition } \\
\text { and missing data? }\end{array}$ & $\begin{array}{l}\text { Did the study perform } \\
\text { appropriate statistical } \\
\text { analyses on potential } \\
\text { confounders? }\end{array}$ & $\begin{array}{l}\text { Is there important } \\
\text { differential attrition and } \\
\text { missing data or overall } \\
\text { high attrition and missing } \\
\text { data? }\end{array}$ & $\begin{array}{l}\text { Were outcomes prespecified } \\
\text { and defined, and ascertained } \\
\text { using accurate methods? }\end{array}$ & Risk of Bias \\
\hline Boyd, 2006 & Yes & No & Yes & No & High \\
\hline Levine, 2016 & No & NA & Unclear & Yes & High \\
\hline Rudolph, 2011 & Yes & No & Unclear & Yes & High \\
\hline Vilke, 1999 & No & NA & Unclear & Yes & High \\
\hline Vilke, 2003 & No & NA & Unclear & Yes & High \\
\hline Wampler, 2011 & No & NA & Unclear & Yes & High \\
\hline
\end{tabular}




\section{Appendix F. Strength of Evidence}

Table F-1. Strength of evidence

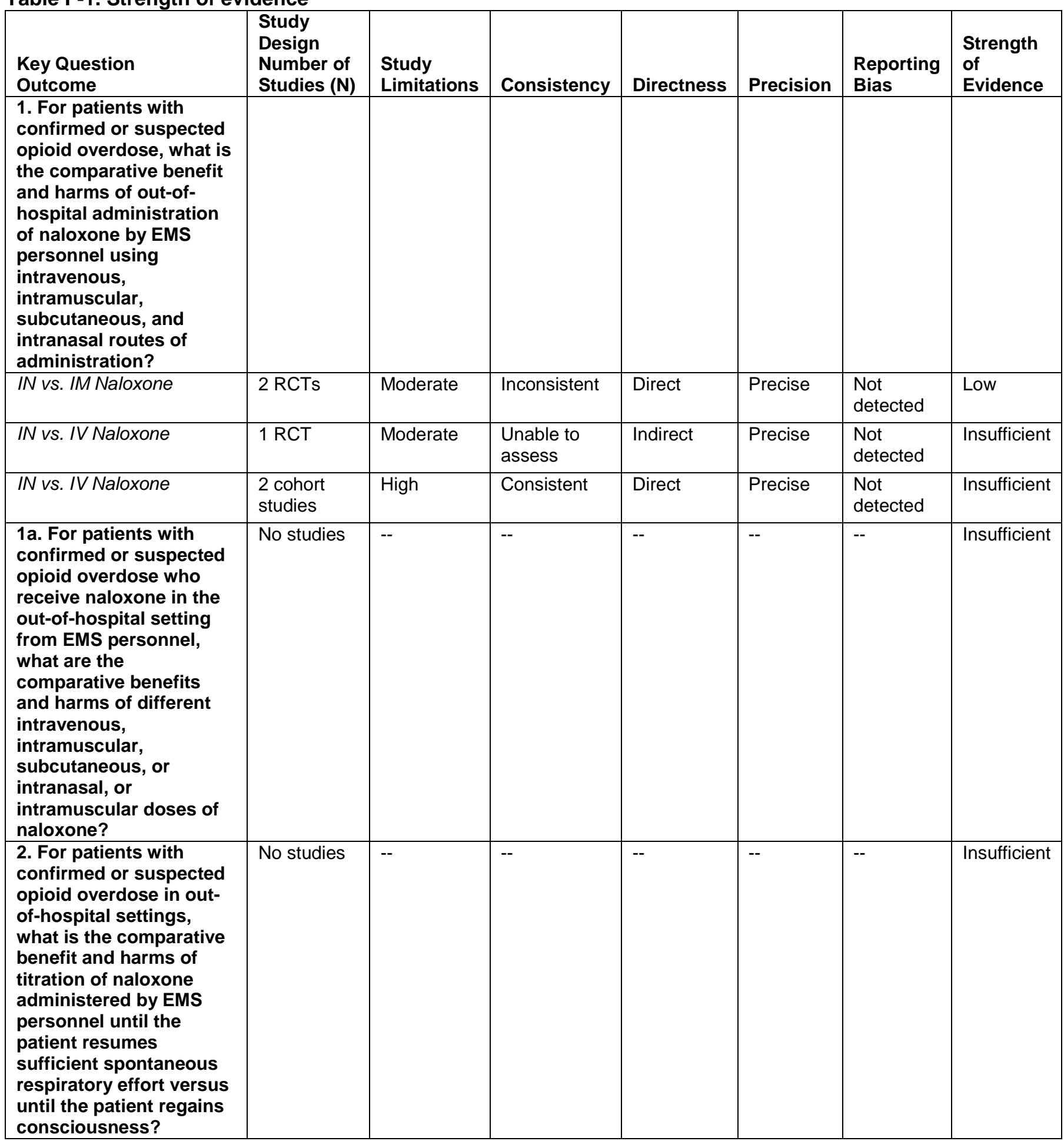




\begin{tabular}{|c|c|c|c|c|c|c|c|}
\hline $\begin{array}{l}\text { Key Question } \\
\text { Outcome }\end{array}$ & $\begin{array}{l}\text { Study } \\
\text { Design } \\
\text { Number of } \\
\text { Studies (N) }\end{array}$ & $\begin{array}{l}\text { Study } \\
\text { Limitations }\end{array}$ & Consistency & Directness & Precision & $\begin{array}{l}\text { Reporting } \\
\text { Bias }\end{array}$ & $\begin{array}{l}\text { Strength } \\
\text { of } \\
\text { Evidence }\end{array}$ \\
\hline $\begin{array}{l}\text { 3. For patients with } \\
\text { confirmed or suspected } \\
\text { opioid overdose in out- } \\
\text { of-hospital settings } \\
\text { treated with multiple } \\
\text { doses of naloxone } \\
\text { (including patients who } \\
\text { do not improve after an } \\
\text { initial dose of intranasal } \\
\text { naloxone), what are the } \\
\text { effects on benefits and } \\
\text { harms of differences in } \\
\text { timing of repeat dosing? }\end{array}$ & No studies & -- & -- & -- & -- & -- & Insufficient \\
\hline $\begin{array}{l}\text { 4. For patients with } \\
\text { confirmed or suspected } \\
\text { opioid overdose in out- } \\
\text { of-hospital settings who } \\
\text { regain sufficient } \\
\text { spontaneous respiratory } \\
\text { effort and are alert and } \\
\text { oriented after naloxone } \\
\text { administration by EMS } \\
\text { personnel, what are the } \\
\text { benefits and harms of } \\
\text { transporting patients to } \\
\text { a health care facility } \\
\text { versus nontransport? }\end{array}$ & & & & & & & \\
\hline $\begin{array}{l}\text { Death, serious adverse } \\
\text { events }\end{array}$ & $\begin{array}{l}6 \\
\text { uncontrolled } \\
\text { studies }\end{array}$ & High & Consistent & Direct & Precise & $\begin{array}{l}\text { Not } \\
\text { detected }\end{array}$ & Insufficient \\
\hline
\end{tabular}

EMS = emergency medical services; IM = intramuscular; IN = intranasal; RCT = randomized controlled trial 